\title{
SYZYGIES OF ABELIAN AND BIELLIPTIC SURFACES IN $\mathbb{P}^{4}$
}

\author{
Alf Aure $\dagger$, Wolfram Decker, Klaus Hulek, Sorin Popescu, Kristian Ranestad
}

\section{Contents}

0 . Introduction

1. The Heisenberg group of level 5

2. Surfaces and syzygies

3. The minimal abelian and bielliptic surfaces

4. Moore matrices

5. Modules obtained by concatenating three Moore matrices

6. Syzygy construction of bielliptic surfaces

7. Non-minimal abelian surfaces obtained via linkage

8. A new family of non-minimal abelian surfaces

9. Degenerations of abelian and bielliptic surfaces

Appendix

\section{INTRODUCTION}

So far six families of smooth irregular surfaces are known to exist in $\mathbb{P}^{4}$ (up to pullbacks of these families by suitable finite covers $\left.\mathbb{P}^{4} \longrightarrow \mathbb{P}^{4}\right)$. These are the elliptic quintic scrolls, the minimal abelian and bielliptic surfaces (of degree 10), two different families of nonminimal abelian surfaces of degree 15 and one family of non-minimal bielliptic surfaces of degree 15. The elliptic quintic scroll is classical. The degree 10 abelian surfaces arise as sections of the Horrocks-Mumford bundle [HM], and the degree 10 bielliptic surfaces were found by Serrano [Ser] using Reider's method [Rei]. It was noticed by Ellingsrud and Peskine (unpublished, compare also [Au]) that the Horrocks-Mumford surfaces can be $(5,5)$-linked to non-minimal abelian surfaces of degree 15 . The other type of non-minimal abelian surfaces of degree 15 was found by the fourth author in his thesis [Po] using the syzygy approach of [DES]. Whereas the abelian surfaces obtained by liaison lie on three quintics, the latter abelian surfaces are contained in one quintic hypersurface only. Finally, the existence of the non-minimal bielliptic surfaces of degree 15 was established in [ADHPR] while studying the geometry of the embedding of the minimal bielliptic surfaces. The unifying element which governs the properties of all these surfaces is their Heisenberg symmetry. In fact, it is only this symmetry which makes it possible to construct the irregular surfaces of degree 15 (where Reider's method does not apply). In all cases every interesting property of these surfaces we know is related to or depends on the presence of the Heisenberg symmetry. We do not know of any irregular surfaces in $\mathbb{P}^{4}$ which do not possess a Heisenberg symmetry 
(again up to pullbacks under finite covers $\mathbb{P}^{4} \longrightarrow \mathbb{P}^{4}$ ), but we also do not know of an a priori reason why such surfaces should not exist.

The main purpose of this paper is to describe the structure of the Hartshorne-Rao modules and the syzygies of the known smooth irregular surfaces in $\mathbb{P}^{4}$. At the same time we explain how to construct these surfaces via syzygies, thus giving a unified construction method for the smooth irregular surfaces known so far. In fact, this means that one can now construct all known smooth non-general type surfaces in $\mathbb{P}^{4}$ via the syzygy approach (compare [DES], [Po], [Sch]). In particular, one can compute explicit equations for all these surfaces with the help of Macaulay [Mac]. We refer to [DES] for remarks concerning these computations in general and to http://yosemite.math.uni-sb.de/software.html for the corresponding programs. In fact, the main results of this paper (6.3 and 6.6) where first predicted and checked by using Macaulay. The degree of the smooth non-general type surfaces in $\mathbb{P}^{4}$ is bounded [EP], and it is conjectured that the precise bound is 15 . The degree 15 surfaces considered in this paper are the only smooth non-general type surfaces known of degree 15 .

The abelian and bielliptic surfaces have in common that either their first Hartshorne-Rao module (in the degree 10 cases) or the dual of their second Hartshorne-Rao module (in the degree 15 cases) has up to twists a minimal free presentation of type

$$
0 \leftarrow M \leftarrow 5 R \stackrel{\alpha_{1}}{\longleftarrow} 15 R(-1) \leftarrow \ldots,
$$

where $R$ is the homogeneous coordinate ring of $\mathbb{P}^{4}$. In fact, by Heisenberg invariance, $\alpha_{1}$ is of type

$$
\alpha_{1}=\left(M_{p_{1}}(y), M_{p_{2}}(y), M_{p_{3}}(y)\right),
$$

where each building block $M_{p_{i}}$ is what we call a Moore matrix (compare Section 4 for a precise definition). The matrices $M_{p_{i}}$ are $5 \times 5$-matrices with linear entries and depend linearly on points $p_{i} \in \mathbb{P}^{4}$. Thus the module $M$ corresponds to a plane $\Pi=<p_{1}, p_{2}, p_{3}>\subset \mathbb{P}^{4}$. The plane $\Pi$ is called the representing plane of $M$. In order to obtain surfaces in this way the representing plane has to be chosen very carefully, in particular the general plane does not give rise to surfaces.

The abelian surfaces are in addition invariant under a certain involution $\iota$ of $\mathbb{P}^{4}$. There are basically only 3 possibilities to construct a module as above which is Heisenberg- and $\iota$ invariant. This can be stated precisely using the eigenspaces $\mathbb{P}_{+}^{2}$ and $\mathbb{P}_{-}^{1}$ of $\iota$ (compare (5.1)). By [De2] the plane $\mathbb{P}_{+}^{2}$ gives the first cohomology module of the Horrocks-Mumford bundle and leads thus to the abelian surfaces of degree 10 (and hence also to the linked abelian surfaces of degree 15). The second of these possibilities, in a special case, leads to the union of two elliptic quintic scrolls (see 6.7). Finally, the third possibility gives rise to the new abelian surfaces of degree 15 found in [Po] (compare Section 8). The bielliptic surfaces are only Heisenberg- but not $\iota$-invariant. On the other hand, we know from [ADHPR] that every minimal and non-minimal bielliptic surface lies on a unique quintic hypersurface, namely either the trisecant scroll of a quintic elliptic scroll or the secant variety of an elliptic normal curve in $\mathbb{P}^{4}$. In fact, every such quintic hypersurface contains 8 bielliptic surfaces paired under $\iota$ and a pencil of abelian surfaces of degree either 10 or 15 arising from the HorrocksMumford bundle. Since the representing plane $\mathbb{P}_{+}^{2}$ of the module of these abelian surfaces can be seen as the plane spanned by the non-trivial 2-torsion points of any given elliptic quintic normal curve, and since there are 8 non-trivial 3-torsion points on such a curve, 
a natural idea for the bielliptic surfaces is to study planes which depend on the 3-torsion points of an elliptic normal curve (compare 6.3 and 6.6). These torsion points are given as the intersection of the curve with a certain hypersurface (see 4.13). For explicit calculations on a computer it is convenient to work over a finite prime field $\mathbb{Z}_{p}$ (compare again [DES]). For this purpose one has to choose the characteristic $p$ such that at least some of the torsion points are rational over $\mathbb{Z}_{p}$.

In the case of abelian surfaces the module $M$ does not determine the surface. It either gives rise to the Horrocks-Mumford bundle (in the degree 10 case) or to certain reflexive sheaves (for the non-minimal abelian surfaces) whose sections give the corresponding surfaces. On the other hand both the minimal and the non-minimal bielliptic surfaces are determined by their module $M$ (see again 6.3 and 6.6).

The building blocks of the syzygies of $M$ are again matrices associated to homomorphisms of Heisenberg modules. The building blocks $L_{q}(y)$ of the first order linear syzygies of $\alpha_{1}$ for example are $5 \times 5$-matrices of a type which we call syzygy matrices. They also depend linearly on parameter points $q \in \mathbb{P}^{4}$.

The paper is organized as follows:

In Section 1 we recall the representation theory which we will need later on. Section 2 contains a quick review of the syzygy approach of [DES], which is in fact an application of Beilinson's theorem [Bei]. In Section 3 we study the Horrocks-Mumford surfaces from a Beilinson point of view, and we determine the shape of the syzygies of the minimal bielliptic surfaces. We are, however, at this point not yet able to determine the differentials of the minimal free resolution of the module $M$ leading to the minimal bielliptic surfaces.

Sections 4, 5 and 6 contain the main new ideas and arguments of this paper. In the sequel we will give a short outline of these sections (see the beginning of Section 4 for more details).

Moore matrices and syzygy matrices make their first appearance in Section 4, where they arise naturally as matrices representing certain Heisenberg invariant homomorphisms coming from the Koszul complex on $\mathbb{P}^{4}$. It is also in this section that we first establish various relations between Moore matrices and geometric objects. Indeed, it is well known and goes back to Bianchi [Bi], Klein [Kl], and others, that Moore matrices are the crucial ingredients in the minimal free resolution of elliptic normal curves in $\mathbb{P}^{4}$. Similarly, syzygy matrices, i.e., matrices of type $L_{q}(y)$, appear in the minimal free resolution of elliptic quintic scrolls (see Lemma 4.4). For certain parameter points the determinants of our matrices define the secant variety of an elliptic normal curve and the trisecant variety of a quintic elliptic scroll resp. (see 4.12). In order to describe the first order linear syzygies of our modules we need to study products of Moore matrices and syzygy matrices. It turns out that these products define an incidence scroll when we restrict the parameter point $p$ of the Moore matrix to an elliptic quintic normal curve. This scroll is closely related to the degree 15 scroll which is the union of the vertices of the rank 3 quadrics through an elliptic quintic normal curve. It is the geometry of these scrolls which will allow us later on to get a hold on the syzygies of the modules $M$. We will also determine the 3 -torsion points and the 6 -torsion points of an elliptic quintic normal curve. For this we need to recall some facts about Shioda's modular surface $S(5)$ and certain maps related to the Horrocks-Mumford bundle.

In Section 5 we study modules obtained by concatenating three Moore matrices, in particular in the cases described in (5.1). We investigate when these modules are artinian (5.5 and 5.6) and we study their linear and quadratic syzygies. Using the results of Section 4 we can 
show that these syzygies correspond to trisecants of certain scrolls. This is another crucial point where we establish a correspondence between algebraic properties of the syzygies and geometric properties of certain scrolls.

Section 6 contains the main results of this paper, namely a complete description of the syzygies of the bielliptic surfaces of degree 10 (see 6.3) and degree 15 (see 6.6).

In Section 7 we briefly review the abelian surfaces of degree 15 which arise by liaison, whereas Section 8 contains a short discussion of the new abelian surfaces of degree 15 which were originally found in [Po]. Finally, in Section 9 we discuss some interesting degenerations of our surfaces. Our considerations underline in particular the fact that the Hilbert scheme is connected $[\mathrm{Ha}]$.

The first author, Alf Aure, died 17.10.94. His death has been a great loss to all of us. While this paper was finished after his death, Alf Aure contributed to all the main ideas and supplied many of the crucial arguments.

Acknowledgement. The authors would like to thank the DFG (Grant Po 514/1-1 and Schwerpunktprogramm "Komplexe Mannigfaltigkeiten", Grant $\mathrm{Hu}$ 337/4-3) and the Center for Advanced Studies, Oslo, for partial support.

\section{The Heisenberg GROUP OF LEVEL 5}

In this section we recall the representation theory of the Heisenberg group $H_{5}$. The geometric significance of $H_{5}$ comes from the fact that its normalizer $N_{5}$ in $S L(5, \mathbb{C})$ is the symmetry group of the Horrocks-Mumford bundle on $\mathbb{P}^{4}[\mathrm{HM}]$, [De1]. In turn, the HorrocksMumford bundle provides a link between some of the $H_{5}$-invariant varieties in $\mathbb{P}^{4}$ which we study in this paper. Some of these varieties are additionally invariant under the involution $\iota \in N_{5}$ defined below. We also recall the representation theory of the subgroup $G_{5} \subset N_{5}$ generated by $H_{5}$ and $\iota$.

Let $V$ be a 5 -dimensional $\mathbb{C}$-vector space with dual bases $e_{0}, \ldots, e_{4}$ of $V$ and $y_{0}, \ldots, y_{4}$ of $V^{*}$. The Heisenberg group of level 5 in its Schrödinger representation is the subgroup $H_{5} \subset S L(V)$ generated by the transformations

$$
\begin{aligned}
& \sigma: e_{i} \rightarrow e_{i-1} \\
& \tau: e_{i} \rightarrow \xi^{i} e_{i}, \quad \xi=e^{2 \pi i / 5} .
\end{aligned}
$$

$H_{5}$ has order 125 and is a central extension

$$
1 \rightarrow \mu_{5} \rightarrow H_{5} \rightarrow \mathbb{Z}_{5} \times \mathbb{Z}_{5} \rightarrow 1
$$

where $\mu_{5}$ is the group of $5^{\text {th }}$ roots of unity. Let $\theta$ be the generator of $\mathrm{Gal}(\mathbb{Q}(\xi): \mathbb{Q})$ given by $\theta(\xi)=\xi^{2}$. The Schrödinger representation of $H_{5}$ on $V$ is an irreducible representation $V_{0}$ of $H_{5}$ and it gives rise to three more: Let $V_{i}$ be the representation given by the composition

$$
H_{5} \stackrel{\theta^{i}}{\rightarrow} H_{5} \rightarrow G L(V), \quad i=\overline{1,3}
$$

$V_{0}, \ldots, V_{3}$ plus the 25 characters of $\mathbb{Z}_{5} \times \mathbb{Z}_{5}$ exhaust the irreducible representations of $H_{5}$. The normalizer $N_{5}$ of $H_{5}$ in $S L(V)$ has order 15,000. In fact,

$$
N_{5} / H_{5} \cong S L\left(2, \mathbb{Z}_{5}\right)
$$


and

$$
N_{5}=H_{5} \rtimes S L\left(2, \mathbb{Z}_{5}\right) \subset S L(V)
$$

is a semi-direct product. Let

$$
G_{5}=H_{5} \rtimes \mathbb{Z}_{2} \subset N_{5}
$$

be the subgroup generated by $H_{5}$ and the involution

$$
\iota: e_{i} \rightarrow e_{-i} .
$$

We recall the the character table of $G_{5}$ from [Ma1]:

\begin{tabular}{|c|c|c|c|}
\hline$\{\alpha\}$ & $C_{m, n}$ & $C_{\alpha}$ & \\
\hline 1 & 1 & 1 & $I$ \\
\hline $5 \theta^{i}(\alpha)$ & 0 & $\theta^{i}(\alpha)$ & $V_{i}$ \\
\hline 1 & 1 & -1 & $S$ \\
\hline $5 \theta^{i}(\alpha)$ & 0 & $-\theta^{i}(\alpha)$ & $V_{i}^{\#}$ \\
\hline 2 & $\xi^{s n+t m}+\xi^{-s n-t m}$ & 0 & $Z_{s, t}$ \\
\hline
\end{tabular}

where $\{\alpha\}$ is the class containing only the central element $\alpha \in \mu_{5}$,

$$
C_{m, n}=\left\{\alpha \xi^{2 m n} \sigma^{m} \tau^{n}, \alpha \xi^{2 m n} \sigma^{-m} \tau^{-n} \mid \alpha \in \mu_{5}\right\}
$$

(there are 12 different $C_{m, n}$ ) and

$$
C_{\alpha}=\left\{\alpha \sigma^{2 m n} \sigma^{m} \tau^{n} \iota \mid m, n \in \mathbb{Z}_{5}\right\}
$$

(there are 5 classes $C_{\alpha}$ ). We denote by $Z$ the direct sum of the 12 different irreducible representations $Z_{s, t}$. If we restrict $Z$ to $H_{5}$ we obtain the direct sum of the non-trivial characters of $H_{5}$. We also need the following formulae from [Ma1]:

$$
\begin{gathered}
V_{i} \otimes V_{i} \cong 3 V_{i+1} \oplus 2 V_{i+1}^{\sharp}, \quad V_{i} \otimes V_{i+1} \cong 3 V_{i+3} \oplus 2 V_{i+3}^{\sharp}, \quad V_{i} \otimes V_{i+2} \cong I \oplus Z \\
\left(V_{i}\right)^{*} \cong V_{i+2}, \quad\left(V_{i}^{\sharp}\right)^{*} \cong V_{i+2}^{\sharp}, \quad V_{i} \otimes S \cong V_{i}^{\sharp}, \quad V_{i} \otimes Z \cong 12 V_{i} \oplus 12 V_{i}^{\sharp},
\end{gathered}
$$

where $i \in \mathbb{Z}_{4}$. For the exterior powers we have

$$
\Lambda^{2} V_{i} \cong 2 V_{i+1}^{\sharp}, \quad \Lambda^{3} V_{i} \cong 2 V_{i+3}^{\sharp}, \quad \Lambda^{4} V_{i} \cong V_{i+2} .
$$

$\mathbb{P}^{4}=\mathbb{P}^{4}(y)=\mathbb{P}(V)$ will stand for the projective space of lines in $V$ and

$$
R=\mathbb{C}\left[y_{0}, \ldots, y_{4}\right]=\bigoplus_{m \geq 0} S^{m} V^{*}
$$

for its homogeneous coordinate ring. If not otherwise mentioned, $R$ carries the action of $G_{5}$ induced by the dual of the Schrödinger representation. The well-known formula for the characters of the symmetric powers

$$
\chi_{s^{m} V}=\sum_{i=1}^{5}(-1)^{i+1} \chi_{s^{m-i} V \otimes \Lambda^{i} V}
$$


yields

$$
\begin{gathered}
H^{0}\left(\mathbb{P}^{4}, \mathcal{O}_{\mathbb{P}^{4}}(1)\right) \cong V_{2}, \quad H^{0}\left(\mathbb{P}^{4}, \mathcal{O}_{\mathbb{P}^{4}}(2)\right) \cong 3 V_{3}, \quad H^{0}\left(\mathbb{P}^{4}, \mathcal{O}_{\mathbb{P}^{4}}(3)\right) \cong 5 V_{1} \oplus 2 V_{1}^{\sharp} \\
H^{0}\left(\mathbb{P}^{4}, \mathcal{O}_{\mathbb{P}^{4}}(4)\right) \cong 10 V_{0} \oplus 4 V_{0}^{\sharp}, \quad H^{0}\left(\mathbb{P}^{4}, \mathcal{O}_{\mathbb{P}^{4}}(5)\right) \cong 6 I \oplus 5 Z .
\end{gathered}
$$

We write

$$
\mathbb{P}_{+}^{2}=\mathbb{P}\left(W_{+}\right), \quad \mathbb{P}_{-}^{1}=\mathbb{P}\left(W_{-}\right) \subset \mathbb{P}(V)
$$

for the eigenspaces of $\iota$. Note that a subgroup of $N_{5}$ isomorphic to the icosahedral group

$$
A_{5} \cong S L\left(2, \mathbb{Z}_{5}\right) /<\iota>
$$

acts on $\mathbb{P}_{+}^{2}$ and $\mathbb{P}_{-}^{1}$ (compare $\left.[\mathrm{BHM} 2,(1.1)]\right)$. We will write $e_{0}=(1: 0: \cdots: 0), \ldots, e_{4}=$ $(0: \cdots: 0: 1)$ for the vertices of the standard simplex in $\mathbb{P}^{4}$ with respect to our fixed basis of $V$.

In Section 4 we consider different $\mathbb{P}^{4}$ 's with coordinates denoted by $y, x, z$ and $w$. Certain natural rational maps between these $\mathbb{P}^{4}$ 's are $G_{5}$-equivariant, if we consider different $H_{5}$ actions as follows: $\mathbb{P}^{4}(y)=\mathbb{P}^{4}\left(V_{0}\right)$ as above, $\mathbb{P}^{4}(x)=\mathbb{P}^{4}\left(V_{1}\right), \mathbb{P}^{4}(z)=\mathbb{P}^{4}\left(V_{0}\right)$ and $\mathbb{P}^{4}(w)=$ $\mathbb{P}^{4}\left(V_{2}\right)$. Note, that a subscheme $\Sigma \subset \mathbb{P}^{4}$ which is $G_{5}$-invariant with respect to one of these actions is also $G_{5}$-invariant with respect to any of the others. In the context of different $\mathbb{P}^{4}$ 's, say e.g. $\mathbb{P}^{4}(y)$ and $\mathbb{P}^{4}(x)$, we will use the following notation: If $\Sigma=\Sigma(y) \subset \mathbb{P}^{4}(y)$ is a subscheme, then let $\Sigma(x) \subset \mathbb{P}^{4}(x)$ denote the same subscheme $\Sigma$ with coordinates $y$ replaced by coordinates $x$.

\section{SURFACES AND SYZYGIES}

In this section we recall from [DES] how to construct surfaces in $\mathbb{P}^{4}$ via the syzygies of their Hartshorne-Rao modules. In fact, this is an application of Beilinson's spectral sequence.

$X$ will always denote a smooth surface in $\mathbb{P}^{4}$ and

- $d$ its degree

- $\pi$ its sectional genus

- $p_{g}$ its geometric genus

- $q$ its irregularity .

In order to construct $\partial_{X}$ and thus $X$ via syzygies one first has to construct the HartshorneRao modules of $X$ :

$$
H_{*}^{i} \partial_{X}=\bigoplus_{m \in \mathbb{Z}} H^{i}\left(\mathbb{P}^{4}, \partial_{X}(m)\right), \quad i=1,2 .
$$

Starting from these graded $R$-modules of finite length one is looking for vector bundles $\mathcal{F}$ and $\mathcal{G}$ on $\mathbb{P}^{4}$ with $\operatorname{rk} \mathcal{G}=\operatorname{rk} \mathcal{F}+1$, and a morphism $\varphi \in \operatorname{Hom}(\mathcal{F}, \mathcal{G})$, which drops rank precisely along $X$. In fact, it is convenient to involve suitable syzygy bundles of the $H_{*}^{i} \partial_{X}$ as direct summands of $\mathcal{F}$ and $\mathcal{G}$. Recall:

Proposition 2.1. Let $M=\bigoplus_{k \in \mathbb{Z}} M_{k}$ be a graded $R$-module of finite length and let

$$
0 \leftarrow M \leftarrow L_{0} \stackrel{\alpha_{1}}{\leftarrow} L_{1} \leftarrow \ldots \stackrel{\alpha_{5}}{\leftarrow} L_{5} \leftarrow 0
$$


be its minimal free resolution. Then for $1 \leq i \leq 3$ the sheafified syzygy module

$$
\mathcal{F}_{i}=S y z_{i}(M)=\left(\operatorname{ker} \alpha_{i}\right)^{\sim}=\left(\operatorname{Im} \alpha_{i+1}\right)^{\sim}
$$

is a vector bundle on $\mathbb{P}^{4}$ with intermediate cohomology

$$
\bigoplus_{k \in \mathbb{Z}} H^{j}\left(\mathbb{P}^{4}, \mathcal{F}_{i}(k)\right) \cong\left\{\begin{array}{rl}
M & j=i \\
0 & j \neq i, \quad 1 \leq j \leq 3 .
\end{array}\right.
$$

Conversely, any vector bundle $\mathcal{F}$ on $\mathbb{P}^{4}$ with this intermediate cohomology is stably equivalent with $\mathcal{F}_{i}$, i.e.,

$$
\mathcal{F} \cong \mathcal{F}_{i} \oplus \mathcal{L}, \quad \mathcal{L} \quad \text { a direct sum of line bundles. }
$$

Example 2.2. The Koszul complex

$$
0 \leftarrow \mathbb{C}(m) \leftarrow R(m) \leftarrow V^{*} \otimes R(m-1) \leftarrow \Lambda^{2} V^{*} \otimes R(m-2) \leftarrow \quad \ldots \quad \leftarrow \Lambda^{5} V^{*} \otimes R(m-5) \leftarrow 0
$$

resolves $\mathbb{C}(m)$, where $\mathbb{C}$ is considered as a graded $R$-module sitting in degree 0 . It follows that

$$
\operatorname{Syz}_{i}(\mathbb{C}(m))=\Omega^{i}(m), 1 \leq i \leq 3,
$$

where $\Omega^{i}=\Lambda^{i} T_{\mathbb{P}(V)}^{*}$. By using the Koszul complex one proves that

$$
\operatorname{Hom}\left(\Omega^{i}(i), \Omega^{j}(j)\right) \cong \Lambda^{i-j} V,
$$

the isomorphism being defined by contraction.

Which syzygy bundles to choose in order to construct a surface $X$ with given invariants comes out by analyzing Beilinson's spectral sequence for $\partial_{X}(m), m=3$ or 4 . Recall:

Theorem 2.3. [Bei] For any coherent sheaf $\mathcal{S}$ on $\mathbb{P}^{4}$ there is a spectral sequence with $E_{1}$-terms

$$
E_{1}^{p q}=H^{q}\left(\mathbb{P}^{4}, \mathcal{S}(p)\right) \otimes \Omega^{-p}(-p)
$$

converging to $\mathcal{S}$, i.e., $E_{\infty}^{p q}=0$ for $p+q \neq 0$ and $\bigoplus E_{\infty}^{-p, p}$ is the associated graded sheaf of a suitable filtration of $\mathcal{S}$.

This fits nicely with the approach via the Hartshorne-Rao modules since the $d_{1}$-differentials

$$
\begin{aligned}
d_{1}^{p q} & \in \operatorname{Hom}\left(H^{q}\left(\mathbb{P}^{4}, \mathcal{S}(p)\right) \otimes \Omega^{-p}(-p), H^{q}\left(\mathbb{P}^{4}, \mathcal{S}(p+1)\right) \otimes \Omega^{-p-1}(-p-1)\right) \\
& \cong \operatorname{Hom}\left(V^{*} \otimes H^{q}\left(\mathbb{P}^{4}, \mathcal{S}(p)\right), H^{q}\left(\mathbb{P}^{4}, \mathcal{S}(p+1)\right)\right)
\end{aligned}
$$

coincide with the natural multiplication maps. In our case this typically means that syzygy bundles of the $H_{*}^{i} \partial_{X}$ will appear in the $E_{2}$-diagram of the spectral sequence. We will interpret one part of Beilinson's spectral sequence for $\partial_{X}(m)$ as the spectral sequence of a vector bundle $\mathcal{F}$, the other part as that of a vector bundle $\mathcal{G}$ with rk $\mathcal{G}=$ rk $\mathcal{F}+1$. The differential between the two parts will define a morphism $\varphi \in \operatorname{Hom}(\mathcal{F}, \mathcal{G})$ whose maximal minors vanish along the desired surface:

$$
0 \rightarrow \mathcal{F} \stackrel{\varphi}{\rightarrow} \mathcal{G} \rightarrow \partial_{X}(m) \rightarrow 0 .
$$

Then we can compute the explicit equations of $X$ from a mapping cone between the minimal free resolutions of $\mathcal{F}$ and $\mathcal{G}$.

Some a priori information on the dimensions $h^{i} \partial_{X}(m)$ can be obtained from RiemannRoch: 
Proposition 2.4. Let $X \subset \mathbb{P}^{4}$ be a smooth surface. Then

$$
\chi\left(\partial_{X}(m)\right)=\chi\left(\mathcal{O}_{\mathbb{P}^{4}}(m)\right)-\left(\begin{array}{c}
m+1 \\
2
\end{array}\right) d+m(\pi-1)-1+q-p_{g}
$$

Moreover one knows:

Proposition 2.5. [DES] Let $X \subset \mathbb{P}^{4}$ be a smooth, non-general type surface which is not contained in any quartic hypersurface. Then we have the following table for the $h^{i} \partial_{X}(m)$ :

\begin{tabular}{c|c|c|c|c|c|c|c}
\multicolumn{9}{l}{} & $i$ \\
\hline & 0 & 0 & 0 & 0 & 0 & 0 & \\
\hline & $N+1$ & $p_{g}$ & 0 & 0 & 0 & 0 & \\
\hline & 0 & $q$ & $h^{2} \partial_{X}(1)$ & $h^{2} \partial_{X}(2)$ & $h^{2} \partial_{X}(3)$ & $h^{2} \partial_{X}(4)$ & \\
\hline & 0 & 0 & 0 & $h^{1} \partial_{X}(2)$ & $h^{1} \partial_{X}(3)$ & $h^{1} \partial_{X}(4)$ & \\
\hline & 0 & 0 & 0 & 0 & 0 & 0 & \\
\hline
\end{tabular}

where

$$
N=\pi-q+p_{g}-1
$$

In the sequel we will represent a zero in a cohomology table by an empty box.

We finally mention two classification results concerning the invariants of those surfaces we are interested in here. Both results are an immediate consequence of adjunction theory [So], [SV].

Proposition 2.6. [Ra, Proposition 9.1] Let $X \subset \mathbb{P}^{4}$ be a smooth surface with $d=10$ and $\pi=6$. Then $X$ is minimal and either abelian, or bielliptic.

For the converse statement compare the next section.

Proposition 2.7. [Po, Lemma 7.1] Let $X \subset \mathbb{P}^{4}$ be a smooth surface with $d=15, \pi=21$, and $\chi\left(\mathcal{O}_{X}\right)=0$. Then $X$ is one of the following:

(i) A ruled surface over an elliptic curve.

(ii) A non-minimal abelian surface.

(iii) A non-minimal bielliptic surface.

In the cases (ii) and (iii) $X$ is embedded by

$$
H=H_{m i n}-\sum_{i=1}^{25} E_{i}
$$

(with obvious notations).

Remark 2.8. It is not known, whether surfaces of type (i) exist. 


\section{THE MiNimAL ABELIAN AND BIELLIPTIC SURFACES}

In this section we review the minimal abelian surfaces in $\mathbb{P}^{4}$ from a syzygy point of view. These surfaces are classified as the smooth zero-schemes of the Horrocks-Mumford bundle in a suitable twist, and they may be constructed from the $H^{1}$-cohomology module of the bundle. It follows, that this module is the first Hartshorne-Rao module for every such surface. Its presentation matrix is known from [De2]. It is the type of this matrix which motivates our considerations later on. We provide further motivation by computing the shape of the minimal free resolution of the first Hartshorne-Rao module of a minimal bielliptic surface in $\mathbb{P}^{4}$. From this computation we may expect and will in fact prove in Section 6, that the presentation matrix is of the same type as above. In this case, however, the surface is uniquely determined by the module.

We will abbreviate HM for Horrocks-Mumford. Let $\mathcal{E}$ be the HM-bundle on $\mathbb{P}^{4}=\mathbb{P}\left(V_{0}\right)$ normalized such that $c_{1}(\mathcal{E})=-1, c_{2}(\mathcal{E})=4$. By its construction in [HM] the bundle is acted on by the normalizer $N_{5}$ of $H_{5}$ in $S L\left(V_{0}\right)$. $\mathcal{E}(3)$ has a four-dimensional space of sections. Since $H^{0} \mathcal{E}(2)=0$ every $0 \neq s \in H^{0} \mathcal{E}(3)$ vanishes along a surface, say $X_{s}$ :

$$
0 \rightarrow \mathcal{O} \stackrel{s}{\rightarrow} \mathcal{E}(3) \rightarrow \partial_{X_{s}}(5) \rightarrow 0
$$

Every such $X_{s}$ is called a $H M$-surface. It is invariant under the action of $G_{5} . \mathcal{E}(3)$ and thus $\partial_{X_{s}}(5)$ are globally generated outside the 25 HM-lines $L_{i j}$ obtained from

$$
L_{00}=\mathbb{P}_{-}^{1}
$$

by translating with the elements of

$$
H_{5} / \mu_{5} \cong \mathbb{Z}_{5} \times \mathbb{Z}_{5}
$$

$L=\bigcup L_{i j}$ is cut out by the $H_{5}$-invariant quintics, i.e., by the elements of

$$
H^{0}\left(\mathcal{O}_{\mathbb{P}^{4}}(5)\right)^{H_{5}} \cong \Lambda^{2} H^{0}(\mathcal{E}(3)) \text {. }
$$

Note that every $\mathrm{H}_{5}$-invariant quintic is in fact also invariant under $\iota$ (an explicit basis of $H^{0}\left(\mathcal{O}_{\mathbb{P}^{4}}(5)\right)^{H_{5}}$ will be given in Section 4). Horrocks and Mumford show that for $s$ generic $X_{s}$ is smooth in the points of $L$ too, and thus it is a minimal abelian surface with $d=10$ and $\pi=6$ (compare Proposition 2.6). Conversely, every minimal abelian surface in $\mathbb{P}^{4}$ arises in this way (up to coordinate transformations). By (3.1) there are $G_{5}$-equivariant isomorphisms

$$
H_{*}^{i} \partial_{X_{*}}(2) \cong H_{*}^{i} \mathcal{E}, \quad i=1,2 \text {. }
$$

In particular, the Hartshorne-Rao modules of $X_{s}$ are independent of $s$. The $H^{2}$-module is just the vector space

$$
H^{2} \partial_{X_{s}} \cong H^{2} \mathcal{E}(-2) \cong 2 S \text {. }
$$

Proposition 3.2. [HM], [De2], [Ma3], [Ma4]. $\quad N=\bigoplus_{k=0}^{3} N_{k}=H_{*}^{1} \mathcal{E}$ is a graded $R$-module with Hilbert function $(5,10,10,2)$, generated by $N_{0} \cong V_{3}$. It has a minimal free resolution of type 


$$
\begin{aligned}
& 0 \leftarrow N \leftarrow 5 R \stackrel{\alpha_{1}}{\leftarrow} 15 R(-1) \nwarrow 10 R(-2) \quad 2 R(-3) \\
& \stackrel{\oplus}{F_{0}} \leftarrow \underset{F_{1}}{\oplus}{ }_{F_{2}} \leftarrow F_{3} \leftarrow 0,
\end{aligned}
$$

where the induced complex

$$
0 \leftarrow H_{*}^{0} \mathcal{E} \leftarrow F_{0}=\underset{15 R(-4)}{\oplus} \nwarrow F_{1}=35 R(-5) \leftarrow F_{2}=20 R(-6) \leftarrow F_{3}=2 R(-8) \leftarrow 0
$$

is the minimal free resolution of $H_{*}^{0} \mathcal{E}$.

Therefore via (3.1) we may compute from $N$ the explicit equations of the minimal abelian surfaces in $\mathbb{P}^{4}$ and their degenerations.

Corollary 3.3. Let $X_{s}$ be a HM-surface. Then $\partial_{X_{s}}$ has syzygies of type

$$
0 \leftarrow \partial_{X_{\diamond}} \leftarrow \underset{15 \mathcal{O}(-6)}{\oplus} \nwarrow_{35 \mathcal{O}(-7) \leftarrow 20 \mathcal{O}(-8)} \nwarrow_{2 \mathcal{O}(-10) \leftarrow 0 .}
$$

In particular, $X_{s}$ is cut out by 3 quintics and 15 sextics. The quintics alone cut out $X_{s}$ and the 25 HM-lines. These are the 6-secant lines to $X_{\text {s. }}$.

To compare later on with the bielliptic surfaces we also mention:

Corollary 3.4. Let $X_{s}$ be a HM-surface. Then:

(i) $\partial_{X}$, has the following cohomology table:

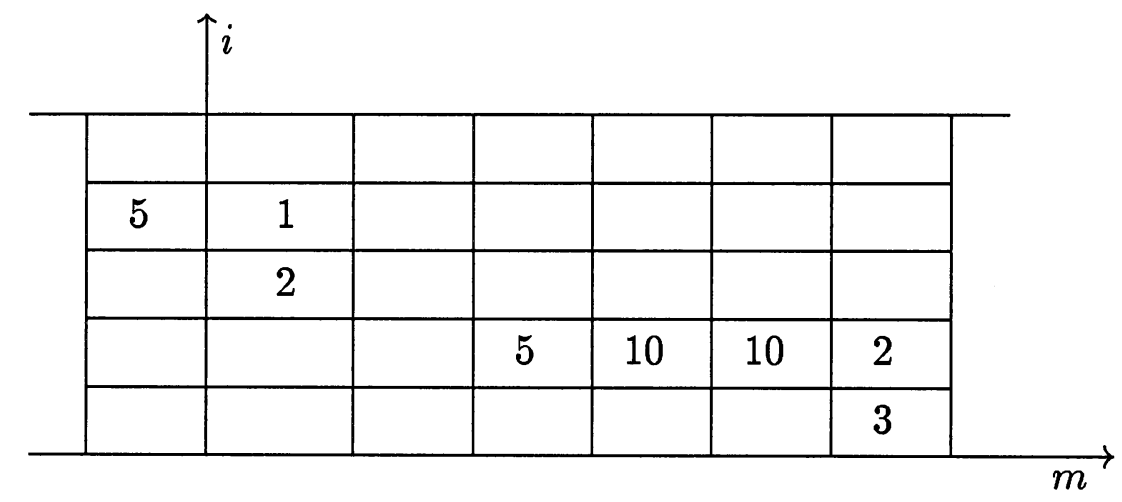

(ii) Beilinson's spectral sequence for $\partial_{X_{s}}(3)$ yields an exact sequence

$$
0 \rightarrow \mathcal{F}=\mathcal{O}(-2) \oplus 2 \Omega^{3}(3) \stackrel{\varphi}{\rightarrow} \mathcal{S}=\mathcal{S}_{y} z_{1}(N(1)) \rightarrow \partial_{X_{\diamond}}(3) \rightarrow 0 .
$$

Proof. (i) We refer to [HM] for the cohomology groups of $\mathcal{E}$. 
(ii) Comparing with the spectral sequence for $\mathcal{E}(1)$ we find that $5 \Omega^{1}(1) \stackrel{d_{1}^{-1,1}}{\rightarrow} 100$ is surjective. Hence also $5 \mathcal{O}(-1) \stackrel{d_{1}^{-4,3}}{\rightarrow} \Omega^{3}(3)$ is surjective with kernel $\mathcal{O}(-2)$. By Beilinson's theorem we obtain a resolution

$$
0 \rightarrow \mathcal{O}(-2) \oplus 2 \Omega^{3}(3) \rightarrow \mathcal{G} \rightarrow \mathcal{J}_{X_{s}}(3) \rightarrow 0
$$

where $\mathcal{G}$ is the kernel

$$
0 \rightarrow \mathcal{G} \rightarrow 5 \Omega^{1}(1) \stackrel{d_{1}^{-1,1}}{\rightarrow} 100 \rightarrow 0
$$

By Proposition 2.1

$$
\mathcal{S}=\mathfrak{S} y z_{1}(N(1))
$$

since both bundles have the same rank and intermediate cohomology.

Remark 3.6. By Beilinson's spectral sequence applied to $\partial_{X_{*}}(4)$ we reobtain the exact sequence (3.1) tensored by -1 .

We will next discuss the shape of the syzygies of the minimal bielliptic surfaces in $\mathbb{P}^{4}$. For this purpose we need to recall from [ADHPR] some of the geometry of the embedding of these surfaces. Let $W$ be a 3-dimensional $\mathbb{C}$-vector space, let

$$
E=E_{\lambda}=\left\{x_{0}^{3}+x_{1}^{3}+x_{2}^{3}+\lambda x_{0} x_{1} x_{2}=0\right\} \subset \mathbb{P}^{2}=\mathbb{P}(W)
$$

be a smooth elliptic curve in the Hesse pencil with origin $p_{0}=(0: 1:-1)$, and let $z_{0}, \ldots, z_{14}$ be the canonical basis of $H^{0} \mathcal{O}_{E}\left(15 p_{0}\right)$. The Heisenberg group $H_{15}$ acts on $H^{0} \mathcal{O}_{E}\left(15 p_{0}\right)$ by

$$
\begin{aligned}
\sigma_{15}: z_{i} \rightarrow z_{i-1}, & \\
\tau_{15}: z_{i} \rightarrow \xi_{15}^{-i} z_{i}, & \xi_{15}=e^{2 \pi i / 15} .
\end{aligned}
$$

We may identify the Heisenberg group $H_{3} \subset S L(W)$ in its Schrödinger representation with the subgroup of $H_{15}$ generated by $\sigma_{3}=\sigma_{15}^{5}$ and $\tau_{3}=\tau_{15}^{5}$. Indeed,

$$
\left[\sigma_{15}^{5}, \tau_{15}^{5}\right]=\xi_{3} \cdot \mathrm{id}, \quad \xi_{3}=e^{2 \pi i / 3} .
$$

So $H_{3} \times H_{3}$ acts naturally on $W_{E}=\mathcal{O}_{E}\left(15 p_{0}\right) \otimes W^{*}$. The center of the diagonal $\Delta \subset H_{3} \times H_{3}$ acts trivially on $W_{E}$. Hence $\mathcal{E}_{E}=W_{E}^{\vee} / \Delta$ is a rank 3 vector bundle over $E / \mathbb{Z}_{3} \times \mathbb{Z}_{3} \cong E$. Consider the corresponding projective quotient map

$$
E \times \mathbb{P}^{2} \rightarrow \mathbb{P}_{E}^{2}=\mathbb{P}\left(\mathcal{E}_{E}\right)
$$

The line bundle $\mathcal{L}^{\prime}=\mathcal{O}_{E}\left(15 p_{0}\right) \otimes \mathcal{O}_{\mathbb{P}^{2}}(1)$ descends to a line bundle $\mathcal{L}$ on $\mathbb{P}_{E}^{2}$. Its sections

$$
\begin{aligned}
& s_{0}=z_{0} \otimes x_{0}+z_{5} \otimes x_{1}+z_{10} \otimes x_{2} \\
& s_{1}=z_{3} \otimes x_{0}+z_{8} \otimes x_{1}+z_{13} \otimes x_{2} \\
& s_{2}=z_{6} \otimes x_{0}+z_{11} \otimes x_{1}+z_{1} \otimes x_{2} \\
& s_{3}=z_{9} \otimes x_{0}+z_{14} \otimes x_{1}+z_{4} \otimes x_{2} \\
& s_{4}=z_{12} \otimes x_{0}+z_{2} \otimes x_{1}+z_{7} \otimes x_{2}
\end{aligned}
$$

are invariant under $\Delta$ and define a basis of $H^{0} \mathcal{L}$. We may identify $H_{5} \subset S L\left(V_{0}^{*}\right)$ in the dual Schrödinger representation with the subgroup of $H_{15}$ generated by $\sigma=\sigma_{15}^{3}$ and $\tau=\tau_{15}^{6}$. Indeed,

$$
\left[\sigma_{15}^{3}, \tau_{15}^{6}\right]=\xi^{-1} \cdot \mathrm{id}
$$


The induced action of $H_{5}$ on $\mathcal{L}^{\prime}$, with $H_{5}$ acting trivially on $\mathcal{O}_{\mathbb{P}^{2}}(1)$, commutes with the action of $H_{3}$. On $H^{0} \mathcal{L}$ it defines the action

$$
\sigma\left(s_{i}\right)=s_{i-1} \quad \tau\left(s_{i}\right)=\xi^{-i} s_{i}
$$

i.e., $H^{0} \mathcal{L}=V_{0}^{*}$ as $H_{5}$-modules. The sections $s_{0}, \ldots, s_{4}$ map $\mathbb{P}_{E}^{2} H_{5}$-equivariantly to a quintic hypersurface which is the trisecant variety

$$
\operatorname{Trisec} S^{2} E \subset \mathbb{P}^{4}=\mathbb{P}\left(V_{0}\right)
$$

$\mathbb{P}_{E}^{2}$ contains a pencil $\left|A_{K}\right|$ of abelian surfaces isogeneous to a product and eight bielliptic surfaces $B_{(i, j)}$, indexed by the non-trivial characters $(i, j) \in \mathbb{Z}_{3} \times \mathbb{Z}_{3} \backslash\{(0,0)\}$. These surfaces are embedded as smooth surfaces with $d=10$ and $\pi=6$. Every minimal bielliptic surface in $\mathbb{P}^{4}$ arises in this way (up to coordinate transformations). By construction, the surfaces $B_{(i, j)}$ are invariant under the Schrödinger action of $H_{5}$ but not under the action of $\iota$. Actually

$$
\iota\left(B_{(i, j)}\right)=B_{(-i,-j)}
$$

Proposition 3.7. Let $B \subset \mathbb{P}^{4}$ be a minimal bielliptic surface embedded as above. Then:

(i) $\partial_{B}$ has the cohomology table

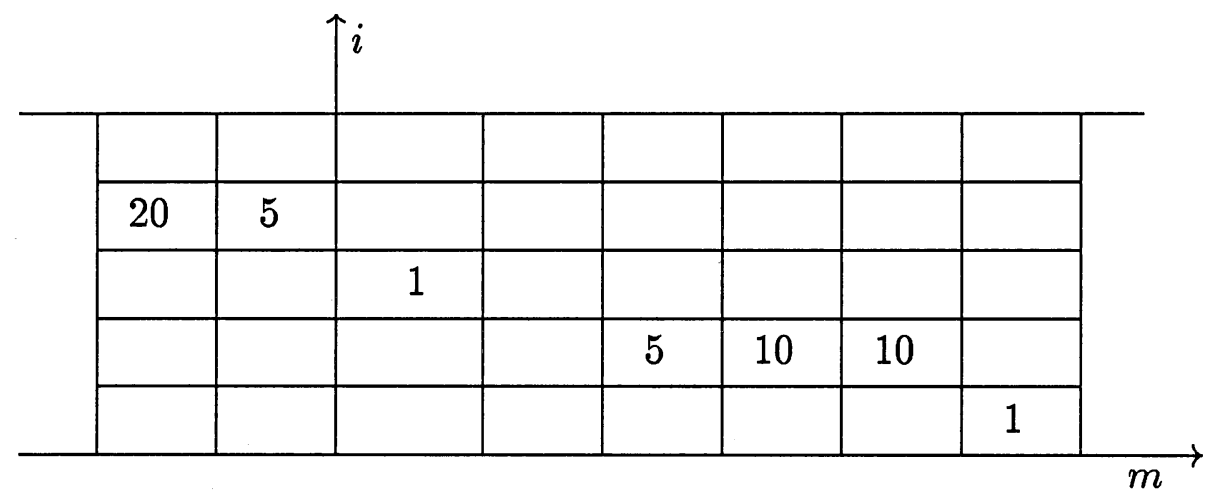

(ii) Beilinson's spectral sequence for $\partial_{B}(3)$ yields an exact sequence

$$
0 \rightarrow \mathcal{F}=5 \mathcal{O}(-1) \oplus \Omega^{3}(3) \rightarrow \mathcal{G}_{B}=\mathcal{S} y z_{1}\left(N^{B}(1)\right) \rightarrow \mathcal{J}_{B}(3) \rightarrow 0
$$

where $N^{B}=\bigoplus_{k=0}^{2} N_{k}^{B}=H_{*}^{1} \partial_{B}(2)$ is a graded $R$-module with Hilbert function $(5,10,10)$, generated by $N_{0}^{B} \cong V_{3}$.

(iii) $N^{B}$ has a minimal free resolution of type

$$
\begin{aligned}
& 0 \leftarrow N^{B} \leftarrow 5 R \leftarrow 15 R(-1) \quad 10 R(-2) \quad R(-3) \\
& \nwarrow \stackrel{\oplus}{\oplus(-3)} \leftarrow \oplus \\
& \stackrel{\oplus}{ } \quad \nwarrow \quad \text { } 5 R(-4) \quad 55 R(-5) \quad 40 R(-6) \leftarrow 10 R(-7) \leftarrow 0 .
\end{aligned}
$$


Equivalently, $\partial_{B}$ has syzygies of type

$$
0 \leftarrow \partial_{B} \leftarrow \underset{25 \mathcal{O}(-6)}{\stackrel{\mathcal{O}(-5)}{\oplus}} \nwarrow 55 \mathcal{O}(-7) \leftarrow 40 \mathcal{O}(-8) \leftarrow 10 \mathcal{O}(-9) \leftarrow 0 .
$$

In particular, $B$ is cut out by 1 quintic and 25 sextics, and it is uniquely determined by $N^{B}$.

Proof. (i) The assertion follows from Riemann-Roch and Proposition 2.5. First notice that $h^{2} \partial_{B}(1)=\chi\left(\partial_{B}(1)\right)=0$. So by taking cohomology in the exact sequences

$$
0 \rightarrow \partial_{B}(m-1) \rightarrow \partial_{B}(m) \rightarrow \partial_{H}(m) \rightarrow 0
$$

associated to the generic hyperplane section $H$ we see that $h^{2} \partial_{B}(m)=0$ for $m \geq 1$. This implies that

$$
h^{1} \partial_{B}(5)=\chi\left(\partial_{B}(5)\right)-1=0,
$$

since $B$ is contained in precisely one quintic hypersurface [ADHPR] .

(ii) Beilinson's spectral sequence for $\partial_{B}(3)$ yields a resolution

$$
0 \rightarrow 5 \mathcal{O}(-1) \oplus \Omega^{3}(3) \rightarrow \mathcal{S}_{B} \rightarrow \partial_{B}(3) \rightarrow 0
$$

where $\mathcal{S}_{B}$ is a kernel

$$
0 \rightarrow \mathcal{G}_{B} \rightarrow 5 \Omega^{1}(1) \rightarrow 100 \rightarrow 0
$$

Then

$$
\operatorname{rank} \mathcal{G}_{B}=10 \leq \operatorname{rank} \delta y z_{1}\left(N_{B}(1)\right) .
$$

By Proposition 2.1 equality holds and

$$
\mathfrak{S}_{B}=\mathcal{S}_{y} z_{1}\left(N^{B}(1)\right)
$$

since both bundles have the same intermediate cohomology. Moreover, Beilinson's spectral sequence represents $\partial_{B}(2)$ as the cohomology of a monad

$$
0 \rightarrow 20 \mathcal{O}(-1) \rightarrow \mathcal{B}=5 \Omega^{3}(3) \oplus \Omega^{2}(2) \rightarrow N_{0}^{B} \otimes \mathcal{O} \rightarrow 0,
$$

and Bott's theorem gives $H_{*}^{1} \mathcal{B}=0$. Hence by taking cohomology in the display of the monad we see that $N_{0}^{B}$ generates $N^{B}$. Part (i) implies that $N^{B}$ has Hilbert function $(5,10,10)$. It remains to show that $N_{0}^{B} \cong V_{3}$. Since $H^{0} \mathcal{O}_{\mathbb{P}^{4}}(2) \cong 3 V_{3}$ this is equivalent to showing that $H^{0} \mathcal{O}_{B}(2) \cong 4 V_{3}$. With the notations introduced after Remark 3.6 it suffices to show that $H^{0}\left(\mathcal{O}_{E}\left(30 p_{0}\right) \otimes \mathcal{O}_{\mathbb{P}^{2}}(2)\right)$, or equivalently that $H^{0} \mathcal{O}_{E}\left(30 p_{0}\right)$ consists of a direct sum of copies of $V_{3}$ only. But since $E$, embedded by $\mathcal{O}_{E}\left(15 p_{0}\right)$ in $\mathbb{P}^{14}$, is projectively normal [Hu1], $H^{0} \mathcal{O}_{E}\left(30 p_{0}\right)$ is a quotient of $H^{0} \mathcal{O}_{\mathbb{P}^{14}}(2) \cong 24 V_{3}$.

(iii) Finally we will compute the shape of the minimal free resolution of $N^{B}$. Since

$$
\operatorname{rank} S y z_{1}\left(N^{B}(1)\right)=10
$$


the syzygies of $N^{B}$ are of type

$$
\begin{aligned}
& 0 \leftarrow N^{B} \leftarrow 5 R \leftarrow 15 R(-1) \\
& 10 R(-2) \\
& \oplus \\
& a R(-3) \\
& \oplus \\
& (25-b+c) R(-4) \\
& \begin{array}{cc}
a R(-3) & b R(-4) \\
\oplus & \oplus \\
\leftarrow c R(-4) \leftarrow & \ldots
\end{array} \\
& \text {... }
\end{aligned}
$$

¿From the mapping cone between the minimal free resolutions of $\mathcal{F}$ and $\mathcal{G}_{B}$ we see that necessarily $a=h^{0} \partial_{B}(5)=1$, hence $b=0$ and $c=h^{0} \partial_{B}(6)-25=\chi\left(\partial_{B}(6)\right)-25=0$ by Riemann-Roch. The remaining graded Betti numbers are uniquely determined by the Hilbert function.

So far we have only discussed the shape of the syzygies of the modules involved. On our way we will explicitly compute the presentation matrices of these modules. For the abelian surfaces this was already done in [De2]. Namely, by using the monad of the HM-bundle it is shown that

$$
\alpha_{1}=\left(\begin{array}{ccccccccccccccc}
y_{0} & 0 & 0 & 0 & 0 & 0 & y_{4} & 0 & 0 & y_{1} & 0 & 0 & y_{3} & y_{2} & 0 \\
0 & 0 & 0 & 0 & y_{2} & y_{1} & 0 & 0 & y_{3} & 0 & 0 & y_{0} & y_{4} & 0 & 0 \\
0 & 0 & 0 & y_{4} & 0 & 0 & 0 & y_{0} & 0 & y_{3} & y_{2} & y_{1} & 0 & 0 & 0 \\
0 & 0 & y_{1} & 0 & 0 & 0 & y_{2} & 0 & y_{0} & 0 & y_{3} & 0 & 0 & 0 & y_{4} \\
0 & y_{3} & 0 & 0 & 0 & y_{4} & 0 & y_{2} & 0 & 0 & 0 & 0 & 0 & y_{1} & y_{0}
\end{array}\right)
$$

is the presentation matrix of $N . \alpha_{1}$ is obtained by concatenating three $5 \times 5$-matrices whose type we will study next.

\section{MOORE MATRICES}

In this section, which is the heart of our paper, we introduce matrices which where first considered by R. Moore [Moo]. These matrices, which we call Moore matrices and syzygy matrices resp., arise naturally from the representation theory of $H_{5}$ and are the crucial building blocks in the minimal free resolutions of $G_{5}$-invariant elliptic normal curves and elliptic quintic scrolls in $\mathbb{P}^{4}$ resp. More general Moore matrices play also a crucial role in [GP1] where they provide equations for $(1, d)$-polarized abelian surfaces. We recall in the sequel some of the known geometry of elliptic curves and their associated varieties, but we also prove some new results which are of a geometric nature. In particular, Proposition 4.13, Lemma 4.18 and Proposition 4.19 are new. Furthermore, we study the interplay between varieties associated to elliptic curves and our matrices. This is new and crucial for everything which comes later. Therefore it is helpful to keep the following geometric picture in mind. In this picture there are two kinds of $G_{5}$-invariant elliptic curves and two kinds of $G_{5}$-invariant elliptic scrolls. The curves are the elliptic normal curves $E$ of degree 5 and certain elliptic curves $E^{\prime}$ of degree 15, while the scrolls are the elliptic quintic scrolls $Q$ and certain singular elliptic scrolls $S$ of degree 15 . These curves and these scrolls all depend on a parameter point in $\mathbb{P}^{1}$ which we omit in our notation at this point, see Propositions 4.2, 4.10,4.3, and 4.16 resp. for precise descriptions. There are certain 3-3 correspondences between these curves and surfaces. Each scroll $Q$ contains three curves $E$ as bisections, and each curve $E$ is contained in three scrolls $Q$. Similarly, each scroll $S$ contains three curves $E^{\prime}$ as bisections, and each curve $E^{\prime}$ lies on three scrolls $S$. On the other hand, each curve $E^{\prime}$ is a section of 
a unique scroll $Q$ and vice versa, and each curve $E$ is the 4-tuple singular locus of a unique scroll $S$ and vice versa. Finally, the union of the curves $E$ is a surface $S_{15}$ of degree 15 with a $G_{5}$-equivariant birational map to a surface $S_{45}$ of degree 45 which is the union of the curves $E^{\prime}$ (Proposition 4.10). The first and the last correspondence is described in Propositions 4.3 and 4.16 resp., the proofs of the others, which are similar, are omitted since we do not need them explicitly. The significance of these curves and surfaces for the Moore matrices $M_{p}$ and the syzygy matrices $L_{q}$ defined below lies in the fact that these matrices have linear entries which depend linearly on a point in $\mathbb{P}^{4}$. When the point $p$ is a general point on a curve $E$, then we may identify the hypersurface defined by $\operatorname{det} M_{p}$ with the secant variety of the curve $E$ and the hypersurface defined by $\operatorname{det} L_{p}$ with the trisecant variety of a scroll $Q$ over $E$. On the other hand, when the point $p$ is a general point on $E^{\prime}$, then we may identify the hypersurface defined by $\operatorname{det} M_{p}$ with the trisecant variety of the unique scroll $Q$ containing $E^{\prime}$ and the hypersurface defined by $\operatorname{det} L_{p}$ with the secant variety of a curve $E$ isomorphic to $E^{\prime}$ via the map from $S_{15}$ to $S_{45}$. This is made precise in Proposition 4.12. The scroll $S$ comes into play when we consider the incidence

$$
I=\left\{(p, q) \mid \text { The quadratic entries of } \quad M_{p} L_{q} \quad \text { all lie in the ideal of } E\right\} .
$$

This incidence variety turns out to be an elliptic scroll over the curve $E$, which is mapped onto a scroll $S$ in the $\mathbb{P}^{4}$ of quadrics in the ideal of $E$ (Lemmas $4.14,4.15$ ).

All surfaces in this paper may be constructed via syzygies from modules presented by a matrix $\alpha_{1}$ which is obtained by concatenating three Moore matrices $M_{p_{i}}, i=\overline{1,3}$. And any five first order linear syzygies of $\alpha_{1}$ will be given by a block of syzygy matrices $L_{q_{i}}$, $i=\overline{1,3}$. To show that the module presented by such a matrix $\alpha_{1}$ gives rise to a surface via a syzygy construction, we need to show that the module is artinian and that it has the correct number of linear and quadratic syzygies. This is done for our choices of points $p_{1}, p_{2}$ and $p_{3}$ in Section 5 using the properties of the Moore matrices and syzygy matrices obtained in this section. For the bielliptic surfaces we choose the points $p_{i}$ on an elliptic curve $E$ and we show in Section 5 how double points and trisecant lines to the scroll $S$ via the above incidence give rise to ten first order linear syzygies for $\alpha_{1}$ in blocks $L_{q_{i j}}, i=\overline{1,3}, j=\overline{1,2}$ (Lemma 5.7 and Proposition 5.8). In fact, given the points $p_{i}$ on $E$ we can identify the corresponding points $q_{i j}$ on a scroll $Q$. More precisely, these points $q_{i j}$ lie on curves of type $E$ or $E^{\prime}$ on $Q$. In Section 6 we use this identification to show that the matrix $\alpha_{1}$ gives rise to a surface on the trisecant variety of a scroll $Q$ or on the secant variety of a curve $E$ (Theorems 6.3 and 6.6). Now the 3-3 correspondences above come into this picture. The module presented by $\alpha_{1}$ depends only on the plane spanned by the points $p_{i}$, the representing plane of this module. For the minimal bielliptic surfaces this plane meets a unique scroll $Q$ in a line and three points, and it is a trisecant plane to all three curves $E$ on this scroll. For the non-minimal bielliptic surfaces the representing plane meets three scrolls $Q$ in a line and three points. These three scrolls intersect along a curve $E$ and the plane is trisecant to this unique curve $E$. For our purposes it is easier to work with a fixed curve $E$, so this 3-3 correspondence will not be explicit in our arguments. Finally, each scroll $Q$ may be identified with the symmetric product $S^{2}(E)$ of one of the curves $E$ (Remark 4.7 , (iii)), so that one may use the arithmetic on $E$ to make computations on $Q$ (Proposition $4.16 \mathrm{ff}$., cf. [Hu1], [ADHPR]).

Matrices of type

$$
M(x, y):=\left(x_{3 i-3 j} y_{3 i+3 j}\right)_{i, j \in \mathbb{Z}_{5}} \quad \text { and } \quad L(z, y):=\left(z_{i-j} y_{2 i-j}\right)_{i, j \in \mathbb{Z}_{5}} \quad \text { resp. }
$$


will be called Moore matrices and syzygy matrices resp. We will also consider these matrices with the $x$ 's, $y$ 's or $z$ 's being replaced by the coordinates of a fixed point in $\mathbb{P}^{4}(x), \mathbb{P}^{4}(y)$ or $\mathbb{P}^{4}(z)$, where we will understand, that these matrices are determined up to scalars only. For example, we will write

$$
M_{p}(y)=M(p, y) \quad \text { and } \quad L_{q}(y)=L(q, y),
$$

if $p=\left(p_{0}: \cdots: p_{4}\right) \in \mathbb{P}^{4}(x)$ and $q=\left(q_{0}: \cdots: q_{4}\right) \in \mathbb{P}^{4}(z)$ are fixed parameter points.

Blocks of such matrices show up if we tensor the Koszul complex resolving $\mathbb{C}(m)$ by $V_{3}$ and decompose into irreducible $H_{5}$-modules:

$$
\ldots \leftarrow V_{3} \otimes R(m) \stackrel{\tilde{M}}{\leftarrow} 5 V_{1} \otimes R(m-1) \stackrel{\tilde{L}}{\leftarrow} 10 V_{0} \otimes R(m-2) \leftarrow \ldots .
$$

More precisely, let us use the notation of Section 1 and choose decompositions of $V_{3} \otimes V_{0}^{*}$ and $V_{1} \otimes V_{0}^{*}$ resp. into irreducible $H_{5}$-modules $V_{1}^{(0)}, \ldots, V_{1}^{(4)}$ of type $V_{1}$ and $V_{0}^{(0)}, \ldots, V_{0}^{(4)}$ of type $V_{0}$ resp. Namely, let

$$
V_{1}^{(k)}=\operatorname{span}\left(f_{0}^{(k)}, \ldots, f_{4}^{(k)}\right) \quad \text { with } \quad f_{i}^{(k)}=e_{2 k+i} \otimes y_{k+i}
$$

and

$$
V_{0}^{(k)}=\operatorname{span}\left(g_{0}^{(k)}, \ldots, g_{4}^{(k)}\right) \quad \text { with } \quad g_{i}^{(k)}=e_{k+i} \otimes y_{2 k+i}
$$

With respect to the first decomposition every element in

$$
\mathbb{P}\left(\operatorname{Hom}_{H_{5}}\left(V_{1} \otimes R(m-1), V_{3} \otimes R(m)\right)\right) \cong \mathbb{P}\left(\operatorname{Hom}_{H_{5}}\left(V_{1}, V_{3} \otimes V_{0}^{*}\right)\right)
$$

is given by the choice of a $H_{5}$-invariant subspace

$$
V^{p} \subset V_{3} \otimes V_{0}^{*} \cong V_{1}^{(0)} \oplus \cdots \oplus V_{1}^{(4)}, \quad p=\left(p_{0}: \cdots: p_{4}\right) \in \mathbb{P}^{4}:
$$

The $i^{\text {th }}$ basis vector of $V^{p}$ is the linear combination of $f_{i}^{(0)}, \ldots, f_{i}^{(4)}$ with $p_{0}, \ldots, p_{4}$ as coefficients. Similarly, we may use the second decomposition to describe the elements in

$$
\mathbb{P}\left(\operatorname{Hom}_{H_{5}}\left(V_{0} \otimes R(m-2), V_{1} \otimes R(m-1)\right)\right) \cong \mathbb{P}\left(\operatorname{Hom}_{H_{5}}\left(V_{0}, V_{1} \otimes V_{0}^{*}\right)\right) .
$$

With respect to these choices and with respect to our fixed basis $e_{i}, i \in \mathbb{Z}_{5}$, of $V_{0}, V_{1}$ and $V_{3}$ we get isomorphisms

$$
\mathbb{P}^{4}(x) \cong \mathbb{P}\left(\operatorname{Hom}_{H_{5}}\left(V_{1} \otimes R(m-1), V_{3} \otimes R(m)\right)\right), \quad p \mapsto M_{p}(y)
$$

and

$$
\mathbb{P}^{4}(z) \cong \mathbb{P}\left(\operatorname{Hom}_{H_{5}}\left(V_{0} \otimes R(m-2), V_{1} \otimes R(m-1)\right)\right), \quad q \mapsto L_{q}(y) .
$$

Let us describe the determinants of our matrices. Following [Moo] one can choose a basis $s_{1}, \ldots, s_{4}$ of $H^{0} \mathcal{E}(3)$ such that

$$
\begin{array}{cc}
\gamma_{0}=s_{1} \wedge s_{2}=5 y_{0} y_{1} y_{2} y_{3} y_{4} & \gamma_{1}=s_{1} \wedge s_{3}=\sum_{i \in \mathbb{Z}_{5}} y_{i} y_{i+2}^{2} y_{i+3}^{2} \\
\gamma_{2}=s_{1} \wedge s_{4}=-\sum_{i \in \mathbb{Z}_{5}} y_{i}^{3} y_{i+2} y_{i+3} & \gamma_{3}=s_{2} \wedge s_{3}=\sum_{i \in \mathbb{Z}_{5}} y_{i}^{3} y_{i+1} y_{i+4} \\
\gamma_{4}=s_{2} \wedge s_{4}=-\sum_{i \in \mathbb{Z}_{5}} y_{i} y_{i+1}^{2} y_{i+4}^{2} & \gamma_{5}=s_{3} \wedge s_{4}=\frac{1}{5} \sum_{i \in \mathbb{Z}_{5}} y_{i}^{5}-\prod_{i \in \mathbb{Z}_{5}} y_{i}
\end{array}
$$


form a basis of

$$
H^{0}\left(\mathcal{O}_{\mathbb{P}^{4}}(5)\right)^{H_{5}} \cong \Lambda^{2} H^{0}(\mathcal{E}(3))
$$

The rational map

$$
\Theta: \mathbb{P}^{4} \rightarrow \mathbb{P}^{5^{*}}=\mathbb{P}\left(H^{0}\left(\mathcal{O}_{\mathbb{P}^{4}}(5)\right)^{H_{5}}\right)^{*}, \quad y \mapsto\left(\gamma_{0}(y): \gamma_{1}(y): \cdots: \gamma_{5}(y)\right)
$$

is well-defined outside the union $L$ of the HM-lines and has as image a smooth hyperquadric $\Omega$ whose dual quadric $\bar{\Omega}=\left\{h_{2} h_{3}-h_{1} h_{4}+h_{0} h_{5}=0\right\} \subset \mathbb{P}^{5}(h)$ may be identified with the Plücker embedding of $\operatorname{Grass}\left(2, H^{0}(\mathcal{E}(3))\right)$ in $\mathbb{P}\left(\Lambda^{2} H^{0}(\mathcal{E}(3))\right)$. By composing $\Theta$ with the Gauss map we obtain

$$
\bar{\Theta}: \mathbb{P}^{4} \backslash L \rightarrow \bar{\Omega} \subset \mathbb{P}\left(H^{0}\left(\mathcal{O}_{\mathbb{P}^{4}}(5)\right)^{H_{5}}\right) \cong \mathbb{P}\left(\Lambda^{2} H^{0}(\mathcal{E}(3))\right) .
$$

The tangent hyperplane $T_{\bar{\Omega}, \bar{\Theta}(y)}$ parametrizes those $H_{5}$-invariant quintics which contain $y \in$ $\mathbb{P}^{4}$, and the quintics in $T_{\bar{\Omega}, \bar{\Theta}(y)} \cap \bar{\Omega}$ correspond to decomposable tensors $s \wedge t \in \Lambda^{2} H^{0}(\mathcal{E}(3))$ with $s(y)=0$. Hence $\bar{\Theta}$ can be seen as the map which associates to a point $y \in \mathbb{P}^{4} \backslash L$ the pencil of sections of $\mathcal{E}(3)$ vanishing in $y$. In particular, $\Theta$ is generically $100: 1$ [Moo]. So via $p \mapsto \Theta^{-1}\left(T_{\Omega, \Theta(p)}\right)$, where $p \in \mathbb{P}^{4}(y)$, we obtain all $H_{5}$-invariant quintics which correspond to decomposable tensors, i.e., those which correspond to points in the Grassmannian of lines in $\mathbb{P}\left(H^{0}(\mathcal{E}(3))\right)$. Every such quintic will be called a $H M$-quintic. The HM-quintics are exactly the determinantal hypersurfaces associated to Moore matrices and syzygy matrices resp.:

Remark 4.1. (i) For Moore matrices we observe that

$$
\begin{aligned}
& \operatorname{det} M(x, y)=\operatorname{det} M(y, x)= \\
& \quad=\gamma_{5}(x) \gamma_{0}(y)-\gamma_{4}(x) \gamma_{1}(y)+\gamma_{3}(x) \gamma_{2}(y)+\gamma_{2}(x) \gamma_{3}(y)-\gamma_{1}(x) \gamma_{4}(y)+\gamma_{0}(x) \gamma_{5}(y) .
\end{aligned}
$$

Therefore $\left\{\operatorname{det} M_{p}(y)=0\right\}=\Theta^{-1}\left(T_{\Omega,\left(\gamma_{0}(p): \gamma_{1}(p): \cdots: \gamma_{5}(p)\right)}\right)$ if $p$ is not on a HM-line.

(ii) For the syzygy matrices we get

$\operatorname{det} L(z, y)=$

$$
=\gamma_{5}(z) \gamma_{0}(y)+\gamma_{2}(z) \gamma_{1}(y)+\gamma_{4}(z) \gamma_{2}(y)+\gamma_{1}(z) \gamma_{3}(y)+\gamma_{3}(z) \gamma_{4}(y)-\gamma_{0}(z) \gamma_{5}(y) .
$$

Hence $\left\{\operatorname{det} L_{q}(y)=0\right\}=\Theta^{-1}\left(T_{\Omega,\left(-\gamma_{0}(q):-\gamma_{3}(q): \gamma_{1}(q): \gamma_{4}(q):-\gamma_{2}(q): \gamma_{5}(q)\right)}\right)$ if $q$ is not on a HMline.

We will show next that the Moore matrices and the syzygy matrices are the crucial building blocks in the minimal free resolutions of $G_{5}$-invariant elliptic normal curves and elliptic quintic scrolls in $\mathbb{P}^{4}$ resp. Let us first recall in the following two propositions a number of standard facts concerning the geometry of these varieties. For proofs we refer to [Hu1], [BHM1], [BHM2] and [Moo].

Proposition 4.2. (i) Elliptic curves with a level-5 structure correspond bijectively to $G_{5}$ invariant elliptic normal curves in $\mathbb{P}^{4}$, i.e., to elliptic normal curves in $\mathbb{P}^{4}$ on which $H_{5}$ operates by translations with 5 -torsion points and $\iota$ as the reflection in the origin given by the intersection of the curve with $\mathbb{P}_{-}^{1}$. There exists a 1-dimensional family $E_{(\lambda: \mu)}$ of $G_{5}$-invariant elliptic normal curves in $\mathbb{P}^{4}$ (possibly degenerate). $E_{(\lambda: \mu)}$ intersects $\mathbb{P}_{-}^{1}$ in $o_{E_{(\lambda: \mu)}}=(\lambda: \mu)$. 
(ii) Let $\Lambda=\left\{(0: 1),(1: 0),\left((1 \pm \sqrt{5}) \xi^{k}: 2\right), k \in \mathbb{Z}_{5}\right\}$. Then for every $(\lambda: \mu) \in \mathbb{P}_{-}^{1} \backslash \Lambda$, the curve $E_{(\lambda: \mu)}$ is smooth. For $(\lambda: \mu) \in \Lambda$, however, $E_{(\lambda: \mu)}$ is a connected cycle of 5 lines, i.e., a pentagon. Notice that we may identify the elements of $\Lambda$ with the vertices of an icosahedron sitting inside $S^{2} \cong \mathbb{P}^{1}$ (cf. [Kl]).

(iii) Two curves of type $E_{(\lambda: \mu)}$ intersect iff the corresponding parameters belong to opposite vertices of the icosahedron. In this case the two singular curves have common vertices and thus form a complete pentagon. Under the action of $N_{5}$ these pentagons are equivalent to the one with vertices $e_{0}, \ldots, e_{4}$, i.e., to $E_{(0: 1)} \cup E_{(1: 0)}$.

(iv) The union $S_{15}=\bigcup_{(\lambda: \mu) \in \mathbb{P}_{-}^{1}} E_{(\lambda: \mu)}$ is an irreducible determinantal surface $S_{15}$ of degree 15. It is smooth outside the 30 vertices of the 6 complete pentagons formed by the singular curves $E_{(\lambda: \mu)},(\lambda: \mu) \in \Lambda$. The normalization of $S_{15}$ is isomorphic to Shioda's modular surface $S(5)$. It thus has a natural structure of an elliptic surface over the modular curve $X(5) \cong \mathbb{P}^{1}$ of level 5 . The smooth fibres are isomorphic to the smooth curves $E_{(\lambda: \mu)}$.

(v) The 5-torsion points in the smooth fibres give rise to 25 sections of $S(5)$ which are mapped 1:1 under $S(5) \rightarrow S_{15}$ onto the Horrocks-Mumford lines. In particular, $X(5)$ may be canonically identified with $L_{00}=\mathbb{P}_{-}^{1}$. We will denote these sections also by $L_{i j},(i, j) \in$ $\mathbb{Z}_{5} \times \mathbb{Z}_{5}$.

(vi) The 3-section of $S(5)$ which intersects each smooth fibre in its non-trivial 2-torsion points is mapped under $S(5) \rightarrow S_{15}$ onto a plane, nodal, irreducible sextic curve $B \subset \mathbb{P}_{+}^{2}$ (Brings curve). The equation of $B$ in $\mathbb{P}_{+}^{2}$ is

$$
y_{0}^{4} y_{1} y_{2}-y_{0}^{2} y_{1}^{2} y_{2}^{2}-y_{0}\left(y_{1}^{5}+y_{2}^{5}\right)+2 y_{1}^{3} y_{2}^{3}=0 .
$$

$B$ has double points at the six points

$$
(1: 0: 0), \quad\left(1: \xi^{i}: \xi^{-i}\right), \quad i \in \mathbb{Z}_{5}
$$

which form the unique minimal $A_{5}$-orbit in $\mathbb{P}_{+}^{2}$.

(vii) Every smooth curve $E_{(\lambda: \mu)}$ meets $B$ in three points. These are the vertices of a triangle tangent to the fixed conic $C_{+}=\left\{y_{0}^{2}+4 y_{1} y_{2}=y_{1}-y_{4}=y_{2}-y_{3}=0\right\} \subset \mathbb{P}_{+}^{2}$. We will identify $X(5) \cong \mathbb{P}_{-}^{1}$ with $C_{+}$via the $A_{5}$-equivariant map $\psi_{5}:(\lambda: \mu) \mapsto\left(2 \lambda \mu: \mu^{2}:-\lambda^{2}\right)$.

Proposition 4.3. (i) For $(\lambda: \mu) \in \mathbb{P}_{-}^{1} \backslash \Lambda$ and $\tau_{i}$ a non-trivial 2-torsion point on $E_{(\lambda: \mu)}$ the translation scroll

$$
\Sigma_{(\lambda: \mu)}\left(\tau_{i}\right):=\bigcup_{p \in E_{(\lambda: \mu)}} \overline{p, p+\tau_{i}} \subset \mathbb{P}^{4}
$$

is a smooth $G_{5}$-invariant elliptic quintic scroll in $\mathbb{P}^{4}$ over the curve $E_{(\lambda: \mu)} /\left\langle\tau_{i}\right\rangle$. $E_{(\lambda: \mu)}$ is contained in $\Sigma_{(\lambda: \mu)}\left(\tau_{i}\right)$ as a bisection of the ruling of this scroll. Conversely, any $G_{5}$ invariant elliptic quintic scroll $\Sigma$ in $\mathbb{P}^{4}$ arises in this way.

(ii) Given $\Sigma$ there exist exactly three pairs $\left(E_{i}, \tau_{i}\right), i=\overline{0,2}$ of elliptic curves and 2-torsion points as above, such that $\Sigma$ is the $\tau_{i}$-translation scroll of $E_{i}$. In particular, $\Sigma$ meets $\mathbb{P}_{+}^{2}$ along the disjoint union of the $\tau_{i}, i=\overline{0,2}$ and the ruling of $\Sigma$ over the origin of the base curve.

(iii) Analogously, given a smooth $G_{5}$-invariant elliptic normal curve $E_{(\lambda: \mu)} \subset \mathbb{P}^{4}$ there exist exactly three $G_{5}$-invariant elliptic quintic scrolls containing it, each two of them meeting transversally along the elliptic curve. Namely, these are the translation scrolls $\Sigma_{(\lambda: \mu)}\left(\tau_{i}\right), \tau_{i}$ a non-trivial 2-torsion point on $E_{(\lambda: \mu)}$. 
We may rephrase these results as follows:

(iv) There exists a 1-dimensional family $Q_{(\lambda: \mu)},(\lambda: \mu) \in \mathbb{P}_{-}^{1}$, of $G_{5}$-invariant elliptic quintic scrolls in $\mathbb{P}^{4}$ (possibly degenerate). The ruling of a smooth $Q_{(\lambda ; \mu)}$ over the origin of the base curve is the line $l_{(\lambda ; \mu)}=\left\{y_{1}-y_{4}=y_{2}-y_{3}=\lambda \mu y_{0}+\mu^{2} y_{2}-\lambda^{2} y_{1}=0\right\} \subset \mathbb{P}_{+}^{2}$. This line is tangent to the conic $C_{+}$at the point $\left(2 \lambda \mu: \mu^{2}:-\lambda^{2}\right)$, which corresponds via $\psi_{5}$ to $(\lambda: \mu) \in \mathbb{P}_{-}^{1} \cong X(5)$.

(v) For every $(\lambda: \mu) \in \mathbb{P}_{-}^{1} \backslash \Lambda$ the surface $Q_{(\lambda: \mu)}$ is smooth. Otherwise $Q_{(\lambda: \mu)}$ is a cycle of 5 planes.

(vi) $Q_{(\lambda: \mu)}$ contains precisely the curves $E_{(\tilde{\lambda}: \tilde{\mu})}$ such that $(\tilde{\lambda}: \tilde{\mu})$ is a root of the polynomial

$$
P_{3,3}=-\lambda^{2} \mu \tilde{\lambda}^{3}+\mu^{3} \tilde{\lambda}^{2} \tilde{\mu}+\lambda^{3} \tilde{\lambda} \tilde{\mu}^{2}+\lambda \mu^{2} \tilde{\mu}^{3}
$$

with $(\lambda: \mu)$ fixed. If $Q_{(\lambda: \mu)}$ is singular then it contains 3 pentagons with 2 of them coming together. Conversely, $P_{3,3}$ determines the surfaces $Q_{(\lambda ; \mu)}$ containing a fixed curve $E_{(\tilde{\lambda}: \tilde{\mu})}$.

Proof. To prove (v) we observe that $Q_{(\lambda ; \mu)}$ is singular iff it contains a pentagon $E_{(\tilde{\lambda} ; \tilde{\mu})}$. The assertion follows from the corresponding smoothness statement for the elliptic curves and (vi) above.

Lemma 4.4. (i) The Moore matrix $M(x, p)$ is skew-symmetric (up to column permutations) for $p=(\lambda: \mu) \in \mathbb{P}_{-}^{1}(y)$ and its $4 \times 4$-Pfaffians define the $G_{5}$-invariant elliptic normal curve $E_{p}=E_{p}(x)$. In particular, $E_{p}$ has a minimal free resolution of type

$$
0 \leftarrow \mathcal{O}_{E_{p}} \longleftarrow \mathcal{O} \stackrel{I_{E_{p}}}{\longleftarrow} V_{3} \otimes \mathcal{O}(-2) \stackrel{M_{(x, p)}}{\longleftarrow} V_{1} \otimes \mathcal{O}(-3) \stackrel{I_{E_{p}}^{t}}{\longleftarrow} \mathcal{O}(-5) \leftarrow 0 .
$$

(ii) For $p=(\lambda: \mu) \in \mathbb{P}_{-}^{1}(y)$ the $4 \times 4$-minors of the syzygy matrix $L(\iota(z), p)$ define the $G_{5}$-invariant elliptic quintic scroll $Q_{p}=Q_{p}(z) \subset \mathbb{P}^{4}(z)$. In particular, $Q_{p}$ has a minimal free resolution of type

$$
0 \leftarrow \mathcal{O}_{Q_{p}} \longleftarrow \mathcal{O} \stackrel{I_{Q_{p}}}{\longleftarrow} V_{1} \otimes \mathcal{O}(-3) \stackrel{L(\iota(z), p)^{t}}{\longleftarrow} V_{0} \otimes \mathcal{O}(-4) \stackrel{[z]^{t}}{\longleftarrow} \mathcal{O}(-5) \leftarrow 0
$$

with $[z]=\left(z_{0}, \ldots, z_{4}\right)$.

Proof. (i) is well-known, compare e.g. [Bi], [Kl].

(ii) Let $p \in \mathbb{P}_{-}^{1}(y)$ and $\tilde{Q}_{p}=\{z \mid \operatorname{rk} L(\iota(z), p) \leq 3\}$. A straightforward calculation shows that the saturated ideal of $\tilde{Q}_{p}$ contains 5 cubics which define a ruled surface of degree 5 (see [Moo, 4.2]). On the other hand, $\left(z_{0}, \ldots, z_{4}\right) \cdot L(\iota(z), p)=0$. Hence $L(\iota(z), p)^{t}$ defines a morphism from $\mathcal{F}=\Omega^{3}(3)$ to $\mathcal{G}=V_{1} \otimes \mathcal{O}$ which drops rank along $\tilde{Q}_{p}$. Altogether $\tilde{Q}_{p}$ is a $G_{5}$-invariant elliptic quintic scroll with the claimed minimal free resolution (compare e.g. [Ok1]). Moreover, $L(\iota(z), p)$ has rank 3 along the line $l_{p} \subset \mathbb{P}_{+}^{2}$ which implies that $\tilde{Q}_{p}=Q_{p}$ by Proposition 4.3, (iv).

Corollary 4.5. Let $p=(\lambda: \mu) \in \mathbb{P}_{-}^{1}$. A set of defining equations for $E_{(\lambda: \mu)}(x)$ is given by

$$
q_{i}^{(\lambda: \mu)}(x)=-\lambda \mu x_{i}^{2}-\mu^{2} x_{i+1} x_{i+4}+\lambda^{2} x_{i+2} x_{i+3},
$$

and a set of defining equations for $Q_{(\lambda: \mu)}(z)$ is given by

$$
\begin{aligned}
c_{i}^{(\lambda: \mu)}(z)= & \lambda^{2} \mu^{2} z_{i}^{3}+\lambda^{3} \mu\left(z_{i+1}^{2} z_{i+3}+z_{i+2} z_{i+4}^{2}\right) \\
& -\lambda \mu^{3}\left(z_{i+1} z_{i+2}^{2}+z_{i+3}^{2} z_{i+4}\right)-\lambda^{4} z_{i} z_{i+1} z_{i+4}-\mu^{4} z_{i} z_{i+2} z_{i+3},
\end{aligned}
$$

where $i \in \mathbb{Z}_{5}$. 
Remark 4.6. As in Lemma 4.4 we obtain: $M(p, y)$ is skew-symmetric for $p=(\lambda: \mu) \epsilon$ $\mathbb{P}_{-}^{1}(x)$ and its $4 \times 4$-Pfaffians define $E_{(\lambda: \mu)}=E_{(\lambda: \mu)}(y)$ in $\mathbb{P}^{4}(y)$. On the other side, $L(q, y)\left(y_{0}, \ldots, y_{4}\right)^{t}=0$ for $q=(\lambda: \mu) \in \mathbb{P}_{-1}^{1}(z)$ and in this case the $4 \times 4$-minors of the matrix $L(q, y)$ define $Q_{(\mu:-\lambda)} \subset \mathbb{P}^{4}(y)$.

Remark 4.7. Let $p=(\lambda: \mu) \in \mathbb{P}_{-}^{1}$.

(i) By applying Beilinson's theorem to $\mathcal{J}_{Q_{p}}(2)$ we get a resolution of type

$$
0 \longleftarrow \mathcal{J}_{Q_{p}}(2) \longleftarrow \Omega^{2}(2) \stackrel{\varphi}{\longleftarrow} 5 \mathcal{O}(-1) \leftarrow 0 .
$$

$\varphi$ is a $1 \times 5$-matrix with entries in $\Lambda^{2} V$ (see Example 2.2). By comparing the mapping cone over $\varphi$ with the minimal free resolution in Lemma 4.4 we see that

$$
\varphi=\left(\mu f^{+}\left(e_{i}\right)+\lambda f^{-}\left(e_{i}\right)\right)_{i \in \mathbb{Z}_{5}},
$$

where $e_{0}, \ldots, e_{4}$ is the chosen basis for $V$ and $f^{ \pm}: V_{1} \rightarrow \Lambda^{2} V_{0}$ are the Horrocks-Mumford maps [HM] defined by

$$
f^{+}\left(\sum x_{i} e_{i}\right)=\sum x_{i} e_{i+2} \wedge e_{i+3} \quad \text { and } \quad f^{-}\left(\sum x_{i} e_{i}\right)=\sum x_{i} e_{i+1} \wedge e_{i+4}
$$

Notice that $f^{ \pm}$correspond to the pencil of trivial characters in $\operatorname{Hom}_{G_{5}}\left(V_{1}, \Lambda^{2} V_{0}^{\sharp}\right) \cong 2 I \oplus$ 2Z.(ii) The Plücker equations for $\mu f^{+}(x)+\lambda f^{-}(x) \in \Lambda^{2} V_{0}$ to be a decomposable tensor, i.e., to correspond to a line $L_{x} \subset \mathbb{P}^{4}$, are just the equations of the elliptic curve $E_{(\lambda: \mu)}$.

(iii) The lines $L_{x}, x \in E_{(\lambda: \mu)}$, are the rulings of the scroll $Q_{(\lambda: \mu)} . L_{o_{(\lambda: \mu)}}$ is exactly $l_{(\lambda: \mu)}$, the ruling over the origin of the base curve. Any scroll $Q_{(\lambda: \mu)}$ may be identified with the symmetric product of an elliptic curve (cf. [BHM2] p.744). Therefore, in our case, $Q_{(\lambda: \mu)}$ may be canonically identified with $S^{2}\left(E_{(\lambda: \mu)}\right)$.

Next we will discuss some facts concerning $S_{15}$ and other projective models of the Shioda modular surface $S(5)$. First we recall from $[\mathrm{BH}]$ a natural choice for a $\mathbb{Q}$-basis of the Néron-Severi group of $S(5)$. As mentioned in Proposition 4.2, (v), $S(5)$ has 25 sections $L_{i j}$, $(i, j) \in \mathbb{Z}_{5} \times \mathbb{Z}_{5}$, which consist of the 5-torsion points in each smooth fibre. Furthermore $S(5)$ has 12 singular fibres of type $I_{5}$. We will order the components $F_{k l}, k=\overline{1,12}$ and $l \in \mathbb{Z}_{5}$ of the singular fibres $F_{k}$ in a coherent way, such that $L_{00} \cdot F_{k 0}=1$ and $F_{k l} \cdot F_{k, l+1}=1$. If $F$ denotes the class of a smooth fibre, then $L_{00}, F$ and $F_{k l}, k=\overline{1,12}, l=\overline{1,4}$, are a basis for $\mathrm{NS}(S(5)) \otimes \mathbb{Q}$ (cf. [BH, Prop.1]). A result of Inoue and Livné (cf. [BH, Theorem 1]) says, that there exists a unique divisor class $I \in \operatorname{Pic}(S(5))$ with $5 I \equiv \sum_{i, j} L_{i j}$. In terms of the above basis it is given by

$$
I=5 L_{00}+24 F-\sum_{k=1}^{12}\left(2 F_{k 1}+3 F_{k 2}+3 F_{k 3}+2 F_{k 4}\right) .
$$

The complete linear system $|I+2 F|$ defines an immersion $\varphi_{1}: S(5) \rightarrow S_{15} \subset \mathbb{P}^{4}[\mathrm{BHM} 1$, Proposition 9], which is nothing else than the normalization morphism described in Proposition 4.2 , (iv). In the sequel we will be mainly concerned with the linear system $\mid 3 I+5 F-$ $\sum P_{k l} \mid$, where $P_{k l}$ are the vertices of the singular fibres of $S(5), k=\overline{1,12}$ and $l \in \mathbb{Z}_{5}$. In [BH, Theorem 4] the following result is proved: 
Proposition 4.8. Let $\tilde{S}(5)$ denote the blow-up of $S(5)$ in the 60 points $P_{k l}$. The linear system $\left|3 I+5 F-\sum P_{k l}\right|$ defines a regular map $\varphi_{4}: \tilde{S}(5) \rightarrow \mathbb{P}^{4}$, which is birational onto a surface $S_{45}$ of degree 45 . The singular fibres of $\tilde{S}(5)$ are mapped to complete pentagons. Two such pentagons coincide if the parameters of the fibres belong to opposite vertices of the icosahedron, and are disjoint otherwise.

The proof in [BH] relies on some properties of the maps $v^{+}$and $v^{-}$introduced in [BHM1]:

$$
v^{+}: V_{1} \rightarrow V_{0}, \quad v^{+}\left(\sum_{i \in \mathbb{Z}_{5}} x_{i} e_{i}\right)=\sum_{i \in \mathbb{Z}_{5}}\left(x_{i+2} x_{i+4}^{2}-x_{i+1}^{2} x_{i+3}\right) e_{i},
$$

and

$$
v^{-}: V_{1} \rightarrow V_{0}, \quad v^{-}\left(\sum_{i \in \mathbb{Z}_{5}} x_{i} e_{i}\right)=\sum_{i \in \mathbb{Z}_{5}}\left(x_{i+1} x_{i+2}^{2}-x_{i+3}^{2} x_{i+4}\right) e_{i} .
$$

$v^{ \pm}$correspond to the pencil of trivial characters in $\operatorname{Hom}_{G_{5}}\left(V_{1}, V_{0} \otimes V_{0}^{\sharp}\right) \cong 2 I \oplus 3 S \oplus 5 Z$. We recall from [BHM1]:

Remark 4.9. Let $(\lambda: \mu) \in \mathbb{P}_{-}^{1}$.

(i) $v^{+}(x)=v^{-}(x)=0$ exactly at the singular points of $S_{15}$.

(ii) If $x \in \mathbb{P}^{4}$ is none of these singular points then $\mu v^{+}(x)-\lambda v^{-}(x)=0$ iff $\mu f^{+}(x)+$ $\lambda f^{-}(x)$ is a decomposable tensor in $\Lambda^{2} V_{0}$. When this occurs the point $\mathbb{P}\left(v^{ \pm}(x)\right)$ lies on the corresponding line $L_{x}$.

(iii) The five cubic equations arising from $\mu v^{+}(x)-\lambda v^{-}(x)=0$ cut out the union of $E_{(\lambda: \mu)}$ and the singular points of $S_{15}$ (in case $E_{(\lambda: \mu)}$ is a pentagon its vertices show up as embedded points). In particular, $v^{+}$and $v^{-}$are proportional exactly over $S_{15}$.

In the sequel we will consider $v^{+}$as the $G_{5}$-equivariant rational map

$$
v^{+}: \mathbb{P}^{4}(x)=\mathbb{P}^{4}\left(V_{1}\right)--\rightarrow \mathbb{P}^{4}(z)=\mathbb{P}^{4}\left(V_{0}\right)
$$

defined in coordinates by $z_{i} \rightarrow x_{i+2} x_{i+4}^{2}-x_{i+1}^{2} x_{i+3}$. Then the base locus of $v^{+}$is the disjoint union of the pentagon $E_{(0: 1)}(x)=\left\{x_{i+1} x_{i+4}=0\right\}_{i \in \mathbb{Z}_{5}}$ (scheme theoretically the vertices show up as embedded points) and the 25 vertices of the other 5 complete pentagons. In particular, $v^{+}$is dominant and a standard computation shows that it is generically $16: 1$. Moreover, since $v^{+}$and $v^{-}$are given by cubic polynomials, and since every linear combination of them vanishes on a fibre $E_{(\lambda: \mu)}$ of $S_{15}$ and on the vertices $P_{k l}$ we have a commutative diagram

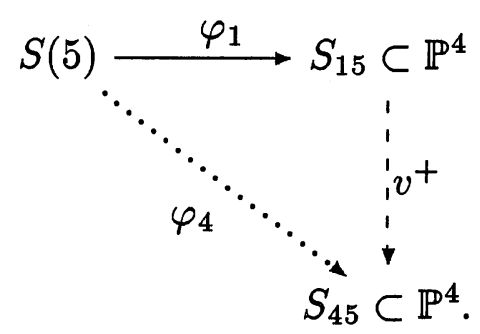


Proposition 4.10. (i) $\varphi_{4}$ maps $L_{00}$ birationally onto the conic $C_{+}=\left\{z_{0}^{2}+4 z_{1} z_{2}=\right.$ $\left.z_{1}-z_{4}=z_{2}-z_{3}=0\right\} \subset \mathbb{P}_{+}^{2}$.

(ii) $v^{+}$maps $\mathbb{P}_{+}^{2}$ onto $\mathbb{P}_{-}^{1}$. The 3 -section $\mathcal{D}$ of $S(5)$ which intersects each smooth fibre in its non-trivial 2 -torsion points is mapped $3: 1$ by $\varphi_{4}$ onto $\mathbb{P}_{-}^{1}$.

(iii) $v^{+}$embeds a curve $E_{(\lambda: \mu)},(\lambda: \mu) \in \mathbb{P}_{-}^{1} \backslash \Lambda$, as a $G_{5}$-invariant elliptic curve $E_{(\lambda: \mu)}^{\prime} \subset \mathbb{P}^{4}$ of degree 15. The curve $E_{(\lambda: \mu)}^{\prime}$ is the section of the scroll $Q_{(\lambda: \mu)}$ which corresponds to the set $\{\{p, q\} \mid 3 p+2 q=0\}$ under the identification $Q_{(\lambda: \mu)}=S^{2}\left(E_{(\lambda: \mu)}\right)$.

Proof. (i) is clear since $\left.v^{+}\right|_{\mathbb{P} 1}$ is the map

$$
v_{\mathbb{P}_{-}^{1}}^{+}:\left(x_{1}: x_{2}\right) \rightarrow\left(2 x_{1}^{2} x_{2}: x_{1} x_{2}^{2}:-x_{1}^{3}:-x_{1}^{3}: x_{1} x_{2}^{2}\right)
$$

(ii) follows readily since $v_{\left.\right|_{\mathbb{P}_{+}^{2}} ^{+}}$is the map

$$
v_{\mathbb{P}_{+}^{2}}^{+}:\left(x_{0}: x_{1}: x_{2}\right) \rightarrow\left(0: x_{1} x_{2}^{2}-x_{0}^{2} x_{2}: x_{1}^{3}-x_{0} x_{2}^{2}: x_{0} x_{2}^{2}-x_{1}^{3}: x_{0}^{2} x_{2}-x_{1} x_{2}^{2}\right),
$$

and since $v^{+}$restricted to the Brings curve $B \subset \mathbb{P}_{+}^{2}$ has as base locus a scheme of length 15 . Indeed, it follows from the above discussion that set-theoretically this base locus coincides with

$$
\mathbb{P}_{+}^{2} \cap\left\{v^{+}=v^{-}=0\right\} \cup\{(0: 0: 1: 1: 0)\},
$$

i.e., that it consists of the double points $e_{0}=(1: 0: 0),\left(1: \xi^{i}: \xi^{-i}\right), i \in \mathbb{Z}_{5}$, of $B$ and the point $(0: 0: 1)$, and that these points count with different multiplicities as follows. The node $e_{0}$ has multiplicity 4 since there are exactly two pentagons, namely $E_{(0: 1)}$ and $E_{(1: 0)}$ having $e_{0}$ as a vertex where two non-trivial 2-torsion points coalesce, while all the other five nodes have multiplicity 2 since for each of them there are precisely two pentagons meeting $\mathbb{P}_{+}^{2}$ transversely in such a point (compare with Lemma 9.1, (ii) for more details). Similarly, $(0: 0: 1)$ counts with multiplicity 1 in the base locus.

(iii) It follows from Remark 4.7 and Remark 4.9 that the image $E_{(\lambda: \mu)}^{\prime}$ of $E_{(\lambda: \mu)}$ under $v^{+}$ is a section of $Q_{(\lambda: \mu)}$. This section meets the ruling over the origin of this scroll in the point $v^{+}((\lambda: \mu))$, i.e., in its point of tangency to the conic $C_{+}$. On the other hand, $E_{(\lambda: \mu)}^{\prime}$ has degree either 15 or 5 by $G_{5}$-invariance. Moreover, it is of numerical class $C_{0}+b f$ with $b=12$ or $b=2$ since it meets the ruling in one point. Here $C_{0}$ is the minimal section and $f$ a ruling. There is no $G_{5}$-invariant curve in the numerical class of $C_{0}+2 f$, thus $E_{(\lambda: \mu)}^{\prime}$ has degree 15 and is of numerical class $C_{0}+12 f$. A $G_{5}$-invariant curve in this class is of type $2 p+3 q=\tau_{i}$ on $S^{2}\left(E_{(\lambda: \mu)}\right), \tau_{i}$ a 2-torsion point on $E_{(\lambda: \mu)}$. By looking again at the intersection point with the ruling over the origin we see that necessarily $\tau_{i}=0$.

For later reference we recall from [ADHPR] the following:

Remark 4.11. For general $(\lambda: \mu) \in \mathbb{P}_{-}^{1}$ the curve $E_{(\lambda: \mu)}^{\prime}$ coincides with the base locus of the pencil $\left|A_{K}\right|$ of degree 10 abelian surfaces on $\operatorname{Trisec} Q_{(\lambda: \mu)}$.

Proposition 4.12. (i) If $p$ is a point on a smooth fibre $E_{(\lambda: \mu)}(x)$ of $S_{15}$ which is not a 5-torsion point, i.e., which does not lie on a HM-line, then the quintic hypersurface $\left\{\operatorname{det} M_{p}(y)=0\right\}$ is the secant variety $\operatorname{Sec} E_{(\lambda: \mu)}$ and $\left\{\operatorname{det} L_{p}(y)=0\right\}$ is the trisecant variety Trisec $Q_{(\mu:-\lambda)}$. 
(ii) If $q$ is a point on a smooth fibre $E_{(\lambda: \mu)}^{\prime}(x)$ of $S_{45}$ which does not lie on a HM-line, then the quintic hypersurface $\left\{\operatorname{det} M_{q}(y)=0\right\}$ is the trisecant variety $\operatorname{Trisec} Q_{(\lambda: \mu)}$ and $\left\{\operatorname{det} L_{q}(y)=0\right\}$ is the secant variety $\operatorname{Sec} E_{(\lambda ; \mu)}$.

Proof. In view of the correspondence described in [ADHPR, Proposition 12] between secant varieties of elliptic normal curves and trisecant varieties of elliptic quintic scrolls, and of the identity

$$
L(\iota(x), u)^{t} \cdot\left(\begin{array}{l}
y_{0} \\
y_{1} \\
y_{2} \\
y_{3} \\
y_{4}
\end{array}\right)=M(x, y) \cdot\left(\begin{array}{l}
u_{0} \\
u_{1} \\
u_{2} \\
u_{3} \\
u_{4}
\end{array}\right)
$$

it is enough to prove only the statements made for matrices of type $M$.

(i) There are 5 independent $H_{5}$-invariant quintic hypersurfaces through a $G_{5}$-invariant elliptic normal curve (cf. [Moo, 2.4]), hence $\Theta$ contracts $E_{(\lambda ; \mu)}(x)$ to a point $P$. It is shown in [Moo, §3] that $\Theta^{-1}\left(T_{\Omega, P}\right)=\operatorname{Sec} E_{(\lambda: \mu)}$. This quintic in turn coincides with $\left\{\operatorname{det} M_{p}(y)=0\right\}$ by Remark 4.1, (i).

(ii) Analogously, one shows that there are precisely $5 \mathrm{H}_{5}$-invariant quintic hypersurfaces through an elliptic curve of type $E_{(\lambda ; \mu)}^{\prime}(x)$. Namely, $E_{(\lambda ; \mu)}^{\prime}(x)$ lies on the scroll $Q_{(\lambda ; \mu)}$ by Proposition 4.10, (iii), and since there are $3 H_{5}$-invariant quintic hypersurfaces through an elliptic quintic scroll [Hu1, VIII.3.5], since $\left|-2 K_{Q_{(\lambda ; \mu)}}\right|$ moves in a pencil [BHM2, Proposition 5.4], and since $h^{1}\left(\mathcal{J}_{Q_{(\lambda ; \mu)}}(5)\right)=0$ the claim follows by taking the $H_{5}$-invariant part of the cohomology of the exact sequence

$$
o \rightarrow \mathcal{J}_{Q_{(\lambda ; \mu)}}(5) \rightarrow \mathcal{J}_{E_{(\lambda ; \mu)}^{\prime}}(5) \rightarrow \mathcal{O}_{Q_{(\lambda ; \mu)}}\left(-2 K_{Q_{(\lambda ; \mu)}}\right) \rightarrow 0 .
$$

Therefore to show (ii) it is enough to check only one point $q$ on $E_{(\lambda: \mu)}^{\prime}(x) \backslash L$. This is done in [ADHPR, Corollary 14] for $q$ chosen as the intersection point of $E_{(\lambda: \mu)}^{\prime}(x)$ and $C_{+}$.

Proposition 4.13. Let $\Gamma=\left\{x_{0}^{2}+\left(x_{1}+x_{4}\right)\left(x_{2}+x_{3}\right)=0\right\}$ be the quadric cone over the conic $C_{+} \subset \mathbb{P}_{+}^{2}$ with vertex $\mathbb{P}_{-}^{1}$. Then $\Gamma$ meets each smooth fibre $E_{(\lambda: \mu)}$ of the surface $S_{15}$ in twice its origin and simply in the non-trivial 3-torsion points.

Proof. First note that $E_{(\lambda ; \mu)}$ is not contained in $\Gamma$. Since $\Gamma$ is singular along $\mathbb{P}_{-}^{1}$ it will be sufficient to show that the non-trivial 3-torsion points of $E_{(\lambda: \mu)}$ lie in $\Gamma$.

For this we use the 4:4 correspondence between $G_{5}$-invariant elliptic normal curves and trisecant varieties of $G_{5}$-invariant elliptic quintic scrolls. Recall from [ADHPR] that a trisecant scroll Trisec $Q$ contains a pencil $\left|A_{K}\right|$ of abelian surfaces. Among these there are precisely 4 translation scrolls coming from the 4 triangles from the Hesse pencil with respect to the twisted $H_{3}$-action in each plane of Trisec $Q$. Clearly, as translation scrolls they are given by a 3 -torsion point on the corresponding elliptic curve. Notice that these 4 elliptic curves do not lie on the scroll $Q$.

Conversely, given an elliptic curve, a 3 -torsion point on it defines a translation scroll of the above type. The eight non-trivial 3 -torsion points on the curve give rise to only 4 translation scrolls, hence the claimed correspondence.

Now both the elliptic scroll $Q$ and the desingularization of the trisecant scroll Trisec $Q$, are projective bundles over an elliptic curve $E$, and the projections are $G_{5}$-equivariant. We 
denote the plane in Trisec $Q$ over the origin $o_{E}$ of $E$ by $\pi_{o_{E}}$. It contains a plane cubic curve of the scroll $Q$ which we denote by $C_{o_{E}}$. Note that $\pi_{o_{E}}$ is also the plane spanned by the origin of $E_{(\lambda: \mu)}$ and a pair of points $(\rho,-\rho)$, where $\rho$ is a non-zero 3-torsion point of $E_{(\lambda: \mu)}$ and that we obtain any such plane in this way.

The proposition now follows from the

Claim. The plane $\pi_{o_{E}}$ is one of the planes of the cone $\Gamma$.

Proof of claim. The plane cubic curve $C_{o_{E}}$ meets each of the three elliptic normal curves on $Q$ in its origin, i.e., in a point on $\mathbb{P}_{-}^{1}$. Thus $\mathbb{P}_{-}^{1}$ is contained in $\pi_{o_{E}}$. On the other side, $C_{o_{E}}$ meets the ruling $l_{o_{E}}$ of $Q$ over $o_{E}$ in its point of tangency to $C_{+}$, hence $\pi_{o_{E}} \cap \mathbb{P}_{+}^{2} \subset C_{+}$and the claim follows.

Later on we will consider $H_{5}$-invariant graded $R$-modules whose presentation matrix is obtained by concatenating three Moore matrices $M_{p_{1}}(y), M_{p_{2}}(y)$ and $M_{p_{3}}(y)$. It will follow from the representation theory described at the beginning of this section that the first order linear syzygies of such modules come in blocks of $L$-matrices. Therefore we are interested in the product matrices

$$
P_{x, z}(y)=M(x, y) L(z, y) .
$$

Each entry of $P_{x, z}(y)$ is a quadric in the span of

$$
y_{i}^{2}, y_{i+1} y_{i+4}, y_{i+2} y_{i+3}
$$

for some $i \in \mathbb{Z}_{5}$ with coefficients that are bilinear forms in $x$ 's and $z$ 's. We collect these forms in the matrix

$$
\left(\begin{array}{ccccc}
x_{0} z_{0} & x_{1} z_{1} & x_{2} z_{2} & x_{3} z_{3} & x_{4} z_{4} \\
x_{1} z_{3}+x_{4} z_{2} & x_{2} z_{4}+x_{0} z_{3} & x_{3} z_{0}+x_{1} z_{4} & x_{4} z_{1}+x_{2} z_{0} & x_{0} z_{2}+x_{3} z_{1} \\
x_{2} z_{1}+x_{3} z_{4} & x_{3} z_{2}+x_{4} z_{0} & x_{4} z_{3}+x_{0} z_{1} & x_{0} z_{4}+x_{1} z_{2} & x_{1} z_{0}+x_{2} z_{3}
\end{array}\right)
$$

which, after column permutations, can be rewritten in a compact way as

$$
T(x, z)=\left(t_{j i}\right)=\left(\begin{array}{c}
x_{3 i} z_{3 i} \\
x_{3 i+1} z_{3 i+3}+x_{3 i+4} z_{3 i+2} \\
x_{3 i+2} z_{3 i+1}+x_{3 i+3} z_{3 i+4}
\end{array}\right)_{i \in \mathbb{Z}_{5}}
$$

The entries of $T$ define a morphism

$$
\Phi_{T}: \mathbb{P}^{4}(x) \times \mathbb{P}^{4}(z) \rightarrow \mathbb{P}^{14}(t)
$$

Let us fix a point $(\lambda: \mu) \in \mathbb{P}_{-}^{1} \backslash \Lambda$. The variety

$$
I_{(\lambda: \mu)}=\left\{(x, z) \mid P_{x, z}(y)=0 \text { for any } y \in E_{(\lambda: \mu)}(y)\right\} \subset \mathbb{P}^{4}(x) \times \mathbb{P}^{4}(z)
$$

parametrizes the product matrices whose entries are in the linear system of quadrics defining the smooth elliptic curve $E_{(\lambda: \mu)}(y) . I_{(\lambda: \mu)}$ will be used in Section 5 to compute the first order linear syzygies of a module as above in the case that all 3 points $p_{1}, p_{2}$ and $p_{3}$ are on the elliptic curve $E_{(\lambda: \mu)}(x)$. For this purpose we will need the following results. 
Lemma 4.14. $I_{(\lambda: \mu)}$ is cut out by the 10 bilinear forms

$$
\lambda x_{i} z_{i}+\mu\left(x_{i+2} z_{i+1}+x_{i+3} z_{i+4}\right)=\mu x_{i} z_{i}-\lambda\left(x_{i+1} z_{i+3}+x_{i+4} z_{i+2}\right)=0, \quad i \in \mathbb{Z}_{5} .
$$

It is an elliptic scroll, which maps by projection to the elliptic curve $E_{(\lambda: \mu)}(x)$ in the first factor (this map defining the ruling) and isomorphically to $Q_{(\lambda: \mu)}(z)$ in the second factor.

Proof. By writing down $P_{x, z}(y)$ explicitly and by comparing with the equations $q_{i}^{(\lambda ; \mu)}(y)$ of $E_{(\lambda: \mu)}(y)$ given in Corollary 4.5 we see that $I_{(\lambda: \mu)}$ is defined by the condition

$$
\left(\begin{array}{ccc}
\lambda & 0 & \mu \\
\mu & -\lambda & 0
\end{array}\right) \cdot T(x, z)=0
$$

i.e., by the 10 equations above. A straightforward calculation shows that the saturation of these forms with respect to $z_{0}, \ldots, z_{4}\left(x_{0}, \ldots, x_{4}\right)$ contains the equations of $E_{(\lambda ; \mu)}(x)$ $\left(Q_{(\lambda: \mu)}(z)\right)$.

Conversely, by plugging in our fixed point $p=(\lambda: \mu) \in \mathbb{P}_{-}^{1}$, we see that $P_{p, z}(p)=0$ iff $z$ is on the line $l_{p}$, the ruling of $Q_{(\lambda ; \mu)}(z)$ over the origin. By $H_{5}$-invariance the image of $I_{(\lambda: \mu)}$ under the projection to $x$ (to $z$ ) contains 25 points (lines) of $E_{(\lambda: \mu)}(x)\left(Q_{(\lambda: \mu)}(z)\right)$. By Bezout this image contains the whole elliptic curve (scroll).

We have shown that $I_{(\lambda ; \mu)}$ is contained in $E_{(\lambda ; \mu)}(x) \times Q_{(\lambda ; \mu)}(z)$ and that it is a smooth elliptic scroll. We now restrict the morphism $\Phi_{T}$ to this scroll. Let $w_{i}=x_{3 i} z_{3 i}, i=\overline{0,4}$, be the entries of the first row of $T$. Then $\Phi_{T}$ and the rational map defined by the $w_{i}$ into $\mathbb{P}^{4}(w)=\mathbb{P}^{4}\left(V_{2}\right)$ coincide on $I_{(\lambda ; \mu)}$, if $\mathbb{P}^{4}(w)$ is suitably embedded in $\mathbb{P}^{14}(t)$. In fact, under the identification of $\mathbb{P}^{14}(t)$ with the space of quadrics in $y$ 's, we embed $\mathbb{P}^{4}(w)$ as the space of quadrics in the ideal of $E_{(\lambda ; \mu)}(y)$. The image of $I_{(\lambda: \mu)}$ in $\mathbb{P}^{4}(w)$ proves to be very singular along the curve $E_{(\lambda: \mu)}(w)$. More precisely, we have:

Lemma 4.15. Let $S_{(\lambda: \mu)}$ be the image of $I_{(\lambda: \mu)}$ under $\Phi_{T}$. Then $S_{(\lambda: \mu)}$ is contained in the linear subspace $\mathbb{P}_{(\lambda: \mu)}^{4}(w) \subset \mathbb{P}^{14}(t)$ which is cut out by the linear forms

$$
\lambda t_{0 i}+\mu t_{2 i}=\mu t_{0 i}-\lambda t_{1 i}=0, \quad i=\overline{0,4} .
$$

In fact, $S_{(\lambda: \mu)}$ is the degree 15 scroll in $\mathbb{P}_{(\lambda: \mu)}^{4}(w)$ defined by the singular lines of the rank 3 quadrics through $E_{(\lambda: \mu)}(w)$.

Proof. Consider the rational map

$$
\Phi_{T_{0}}: \mathbb{P}^{4}(x) \times \mathbb{P}^{4}(z) \rightarrow \mathbb{P}_{(\lambda: \mu)}^{4}(w) \subset \mathbb{P}^{14}(t),
$$

defined by the entries of the first row of $T$. Clearly, the restrictions of $\Phi_{T}$ and $\Phi_{T_{0}}$ to $I_{(\lambda: \mu)}$ coincide. The first assertion of the lemma follows. For the second one we first remark that $\Phi_{T_{0}}$ maps the ruling $\{p\} \times l_{p}(z)$, where $p=(\lambda: \mu), 1: 1$ to $\mathbb{P}_{-}^{1}(w)$. Thus $S_{(\lambda: \mu)}$ is two-

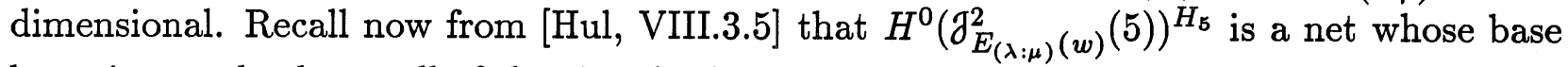
locus is exactly the scroll of the singular lines of the rank 3 quadrics through $E_{(\lambda: \mu)}(w)$. So 
it suffices to show that $S_{(\lambda: \mu)}$ is contained in every $H_{5}$-invariant quintic which is singular along $E_{(\lambda: \mu)}(w)$. By [Hu1, p.82] a basis for these quintics is given by

$$
\begin{aligned}
& Q_{0}(w)=\sum_{i=0}^{4} w_{i} q_{i}^{2} \\
& Q_{1}(w)=\sum_{i=0}^{4} w_{i} q_{i+1} q_{i+4} \\
& Q_{2}(w)=\sum_{i=0}^{4} w_{i} q_{i+2} q_{i+3}
\end{aligned}
$$

where $q_{i}=q_{i}^{(\lambda: \mu)}(w)$ are the equations of $E_{(\lambda: \mu)}(w)$. A straightforward calculation shows that for all $i$

$$
Q_{i}\left(x_{0} z_{0}, x_{3} z_{3}, x_{1} z_{1}, x_{4} z_{4}, x_{2} z_{2}\right)=0
$$

modulo the equations of $I_{(\lambda: \mu)}$.

We recall the geometry of the degree 15 scroll defined by the singular lines of the rank 3 quadrics through an elliptic normal curve in $\mathbb{P}^{4}$.

Proposition 4.16. (i) The scroll $S_{(\lambda: \mu)}$ is a birational image of the symmetric product $S^{2}\left(E_{(\lambda: \mu)}\right)$ via the identification map which associates to a proper secant line $\overline{p q}$ of $E_{(\lambda: \mu)}$ the point of intersection of this secant with the singular line of the rank 3 hyperquadric uniquely determined by the fact that it contains the curve and that its singular locus meets $\overline{p q}$ outside the curve.

(ii) $S_{(\lambda: \mu)}$ is 4-tuple along $E_{(\lambda: \mu)}$ and smooth elsewhere.

(iii) The union $X_{p+q}$ of all the secants of $E_{(\lambda: \mu)}$ spanned by points which add up to $p+q$ in the group law is a smooth rational cubic scroll, whose directrix $d_{p+q}$ is a ruling in $S_{(\lambda: \mu)}$. As a ruling in the symmetric product $S^{2}\left(E_{(\lambda: \mu)}\right)$ this is the ruling over the point $p+q$ on the base curve. This ruling hits the curve $E_{(\lambda: \mu)} \subset \mathbb{P}^{4}$ in exactly one point corresponding to $-2(p+q)$.

(iv) The curve $\{\{p, q\} \mid 3 p+2 q=0\}$ on the symmetric product $S^{2}\left(E_{(\lambda: \mu)}\right)$ is mapped $4: 1$ onto the curve $E_{(\lambda: \mu)} \subset S_{(\lambda: \mu)}$. The image of $\{p, q\}$ is the intersection of the secant $\overline{p q}$ with $d_{p+q}$.

Proof. See [Hu1], [BHM1], [BHM2].

Remark 4.17. (i) The scroll $S_{(\lambda: \mu)}$ contains three elliptic curves, which on the symmetric product $S^{2}\left(E_{(\lambda: \mu)}\right)$ are numerically equivalent to the anticanonical divisor. Namely, these can be identified with the set of unordered pairs of points $\left\{\{p, q\} \mid p=q+\tau_{i}\right\}$ on $E_{(\lambda: \mu)}$, $\tau_{i}$ a non-trivial 2-torsion point on $E_{(\lambda: \mu)}$. The group law is given by $\{p, q\}+\{r, s\}=$ $\{p+r, q+r\}=\{p+s, q+s\}$, which is well-defined since $p-q=r-s=\tau_{i}$ is 2-torsion.

(ii) Through any point of $E_{(\lambda: \mu)}$ there are exactly four rulings of $S_{(\lambda: \mu)}$. In particular, the rulings through the origin of $E_{(\lambda: \mu)}$ correspond to the directrices $d_{\tau_{i}}, \tau_{i}$ a 2-torsion point on $E_{(\lambda: \mu)}$. 
Lemma 4.18. Let $\tau_{i}, i=\overline{1,3}$ be the non-trivial 2-torsion points of $E_{(\lambda: \mu)}$ and let $\Pi_{\tau_{1} \tau_{2}}$ be the plane spanned in $\mathbb{P}^{4}$ by the directrices $d_{\tau_{1}}$ and $d_{\tau_{2}}$. Then $\Pi_{\tau_{1} \tau_{2}}$ meets $S_{(\lambda: \mu)}$ in $d_{\tau_{1}}, d_{\tau_{2}}$ and the points corresponding on the symmetric product to $\left\{\{p, q\} \mid 3(p+q)=p-q=\tau_{3}\right\}$. In particular:

(i) There are exactly 8 rulings not contained in $\Pi_{\tau_{1} \tau_{2}}$ which meet this plane. These are the rulings over the points $x \in E_{(\lambda: \mu)}$ with $3 x=\tau_{1}+\tau_{2}, x \neq \tau_{3}$.

(ii) These rulings meet the plane in non-trivial 6-torsion points of the numerically anticanonical curve on $S_{(\lambda: \mu)}$ corresponding to $\tau_{3}$ as in Remark 4.17, (i).

Proof. Assume that the plane $\Pi_{\tau_{1} \tau_{2}}$ and the ruling over a point $x \in E_{(\lambda: \mu)}$ intersect. Let $H$ be the hyperplane spanned by $\Pi_{\tau_{1} \tau_{2}}$ and this ruling. The curve $E_{(\lambda: \mu)}$ hits this ruling in the point $-2 x$ and the other two rulings in the origin. Therefore we may write

$$
H \cap E_{(\lambda: \mu)}=o_{E_{(\lambda: \mu)}}+(-2 x)+a+b+c .
$$

The chosen 3 rulings are directrices of rational cubic scrolls which contain the elliptic curve, and $H$ intersects each of these scrolls along their directrix and two rulings. From Proposition 4.16, (iii) we get the following relations:

On the scroll with directrix over $\tau_{1}$

$$
a+b=c+(-2 x)=\tau_{1}
$$

On the scroll with directrix over $\tau_{2}$

$$
a+c=b+(-2 x)=\tau_{2}
$$

Note that we have essentially defined the points $a, b, c$ via these relations, hence for the scroll over $x$ we necessarily get

$$
b+c=a+o_{E_{(\lambda: \mu)}}=x .
$$

Adding the second relations in each case we get $a+b+c+(-4 x)=x+\tau_{1}+\tau_{2}$, and since $a+b+c+(-2 x)=0$ we deduce $3 x=\tau_{3}$.

Let us consider now the hyperplane $H^{\prime}$ spanned by $\Pi_{\tau_{1} \tau_{2}}$ and the ruling in the rational scroll over the directrix of $x$. This ruling hits the curve in points $p$ and $q$ say, and thus we can write $H^{\prime} \cap E_{(\lambda: \mu)}=o_{E_{(\lambda ; \mu)}}+p+q+a+b$. As above we get that $a+p=b+q=\tau_{1}$ and $a+q=b+p=\tau_{2}$, which implies $p-q=\tau_{3}$. The rest of the lemma follows now from Remark 4.17, (i).

We will finally determine the 6 -torsion points of a $G_{5}$-invariant elliptic normal curve in $\mathbb{P}^{4}$. For this purpose we will again use the rational map $v^{+}: \mathbb{P}^{4}(x)--\rightarrow \mathbb{P}^{4}(z)$, and the morphism $\Phi_{T}$. Notice that the map

$$
\mathbb{P}^{4}(x)=\mathbb{P}^{4}\left(V_{1}\right)^{--\rightarrow} \mathbb{P}^{4}(w)=\mathbb{P}^{4}\left(V_{2}\right)
$$

defined in coordinates by $w_{2 i} \rightarrow x_{i}\left(x_{i+2} x_{i+4}^{2}-x_{i+1}^{2} x_{i+3}\right)$ is $G_{5}$-equivariant. It corresponds to a trivial character in $\operatorname{Hom}_{G_{5}}\left(V_{1}, V_{0} \otimes V_{3}^{\sharp}\right) \cong 2 I \oplus 3 S \oplus 5 Z$. 
Proposition 4.19. Let $(\lambda: \mu) \in \mathbb{P}_{-}^{1} \backslash \Lambda$.

(i) The graph $\Gamma_{(\lambda ; \mu)} \subset \mathbb{P}^{4}(x) \times \mathbb{P}^{4}(z)$ of the restriction of $v^{+}$to the elliptic normal curve $E_{(\lambda: \mu)}$ is contained in the incidence scroll $I_{(\lambda: \mu)}$.

(ii) $\Phi_{T}$ maps the graph $\Gamma_{(\lambda ; \mu)} 4: 1$ onto the curve $E_{(\lambda: \mu)}(w) \subset \mathbb{P}_{(\lambda: \mu)}^{4}(w)$. Moreover, the composition

$$
\Phi_{T} \circ\left(\operatorname{id}_{E_{(\lambda ; \mu)}(x)} \times\left. v^{+}\right|_{E_{(\lambda ; \mu)}(x)}\right): E_{(\lambda: \mu)}(x) \rightarrow E_{(\lambda: \mu)}(w)
$$

acts as multiplication by 2 in the group law of the elliptic curve $E_{(\lambda: \mu)}$.

iii) The octic hypersurface $\left\{w_{0}^{2}+\left(w_{1}+w_{4}\right)\left(w_{2}+w_{3}\right)=0\right\}$, where $w_{2 i}=x_{i}\left(x_{i+2} x_{i+4}^{2}-\right.$ $\left.x_{i+1}^{2} x_{i+3}\right), i \in \mathbb{Z}_{5}$, meets each smooth fiber of $S_{15} \subset \mathbb{P}^{4}(x)$ in twice the 2-torsion points and simply in the remaining 6-torsion points .

Proof. (i) One can easily check that

$$
\left(\begin{array}{ccc}
\lambda & 0 & \mu \\
\mu & -\lambda & 0
\end{array}\right) \cdot T\left(x, v^{+}(x)\right)=0
$$

modulo the equations $q_{i}^{(\lambda: \mu)}, i \in \mathbb{Z}_{5}$, of $E_{(\lambda: \mu)}(x)$. Hence we deduce from the proof of Lemma 4.14 that the graph $\Gamma_{(\lambda: \mu)}$ is contained in the incidence scroll $I_{(\lambda: \mu)}$.

(ii) By Lemma 4.15 the image of $I_{(\lambda: \mu)}$ under $\Phi_{T}$ is the singular scroll $S_{(\lambda: \mu)}$. Therefore all claims in (ii) follow from the fact that through any point $p$ of $E_{(\lambda: \mu)}(w)$ there pass exactly four rulings of $S_{(\lambda: \mu)}(w)$. Namely, these are the directrices $d_{q}$ of the cubic scrolls $X_{q}$ with $2 q=-p$ in the group law (cf. Proposition 4.16, (iii) and (iv), and Remark 4.17, (ii)).

(iii) is then clear from Proposition 4.13.

For the next section we summarize some of the results of 4.14-4.19. First these results give rise to the diagrams

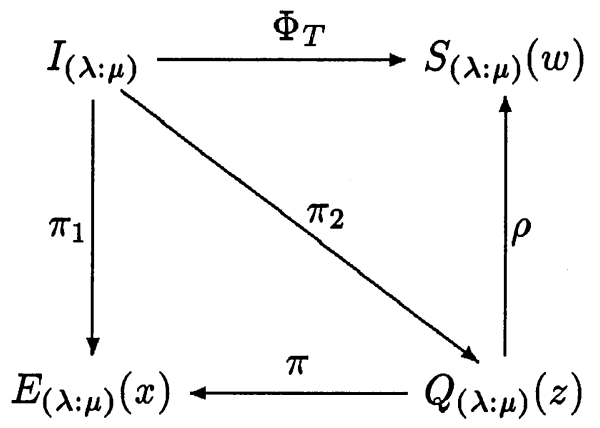

and

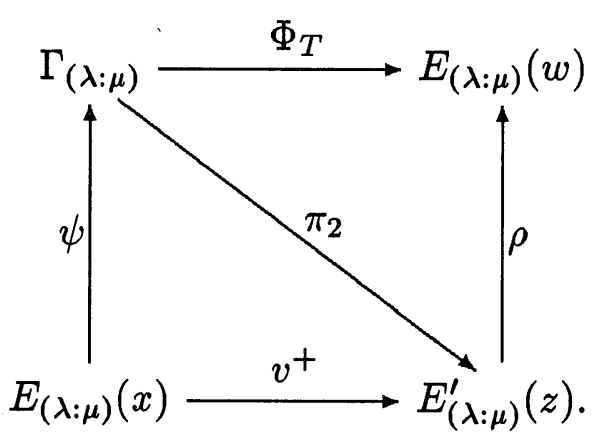


The varieties of the second diagram are subvarieties of the corresponding varieties of the first diagram. In the first diagram $\rho=\Phi_{T} \circ \pi_{2}^{-1}$, and in the second diagram

$$
\psi=\left(\operatorname{id}_{E_{(\lambda ; \mu)}(x)} \times\left. v^{+}\right|_{E_{(\lambda ; \mu)}(x)}\right) .
$$

The maps $\Phi_{T}, \pi_{2}$ and $\rho$ of the second diagram are restrictions of those in the first diagram, while $\psi$ and $v^{+}$are sections of the corresponding maps in the first diagram. 4.14, 4.15, 4.16 and 4.19 say that both diagrams commute. In the next section we will need to know which pairs of points $(p, q) \in I_{(\lambda: \mu)}, p \in E_{(\lambda: \mu)}, q \in Q_{(\lambda: \mu)}$ are mapped by $\Phi_{T}$ to the points of $E_{(\lambda: \mu)}(w)$ and to the plane $\Pi_{\tau_{1} \tau_{2}}$ of Lemma 4.18. Since $v^{+}$is an isomorphism we can identify points on $E_{(\lambda: \mu)}^{\prime}(z)$ with points on $E_{(\lambda: \mu)}(x)$.

Remark 4.20. Under the identification of $Q_{(\lambda: \mu)}$ with $S^{2}\left(E_{(\lambda: \mu)}\right)$ each numerically anticanonical curve on $S^{2}\left(E_{(\lambda: \mu)}\right)$ corresponds to one of the three $G_{5}$-invariant elliptic quintic curves on $Q_{(\lambda: \mu)}$. The curve corresponding to $\left\{\{p, q\} \mid p=q+\tau_{i}\right\}$ will be denoted by $E_{(\lambda: \mu)}\left(\tau_{i}\right)$.

Corollary 4.21. (i) The map $\rho$ of the second diagram is $4: 1$ and acts as multiplication by 2 in the group law of the elliptic curve $E_{(\lambda: \mu)}^{\prime}(z)$.

(ii) The HM-lines in $\mathbb{P}^{4}(z)$ are proper 3-secants to $Q_{(\lambda: \mu)}(z)$. In fact, they are all 3-secants to the curve $E_{(\lambda: \mu)}^{\prime}(z)$ and intersect the curve in the 10-torsion points which are different from the 5-torsion points.

(iii) The numerically anticanonical curve $E_{(\lambda: \mu)}\left(\tau_{3}\right) \subset Q_{(\lambda: \mu)}(z)$ is mapped by $\rho$ isomorphically to an elliptic curve on $S_{(\lambda: \mu)}(w)$ which meets the plane $\Pi_{\tau_{1} \tau_{2}}$ of Lemma 4.18 in 8 non-trivial 6-torsion points.

Proof. (i) follows from 4.14 and 4.19. (ii) follows from 4.10. (iii) follows from and 4.18 .

\section{Modules obtained by Concatenating three Moore matrices.}

In this section we use the results of Section 4 to analyse graded $R$-modules whose presentation matrix is a block of three Moore matrices. We are particularly interested in those modules listed in (5.1). We check under which conditions the modules in (5.1) are artinian and we compute their linear and quadratic syzygies. This provides enough information for the following sections, where we will construct surfaces from these modules and determine their syzygies completely.

Let $\Pi=<p_{1}, p_{2}, p_{3}>\subset \mathbb{P}^{4}(x)$ be a plane, let

$$
\alpha_{1}=\left(M_{p_{1}}(y), M_{p_{2}}(y), M_{p_{3}}(y)\right),
$$

and let $M=\bigoplus_{k \geq 0} M_{k}$ be the $H_{5}$-invariant graded $R$-module presented by $\alpha_{1}$ :

$$
0 \leftarrow M \leftarrow V_{3} \otimes R \stackrel{\alpha_{1}}{\longleftarrow} 3 V_{1} \otimes R(-1)
$$

We call $\Pi$ the representing plane of $M$. The minimal free resolution of $M$ is of type

$$
0 \leftarrow M \leftarrow F_{0,0} \otimes R \stackrel{\alpha_{1}}{\leftarrow} F_{1,1} \otimes R(-1) \stackrel{\alpha_{2}}{\leftarrow} \bigoplus_{l \geq 2} F_{2, l} \otimes R(-l) \stackrel{\alpha_{3}}{\leftarrow} \ldots \stackrel{\alpha_{5}}{\leftarrow} \bigoplus_{l \geq 5} F_{5, l} \otimes R(-l) \leftarrow 0
$$


with $F_{0,0}=M_{0}=V_{3}$ and $F_{1,1}=3 V_{1}$. The syzygies $F_{k, k+l}$ are canonically isomorphic to the cohomology groups of the Koszul complexes

$$
\cdots \leftarrow \Lambda^{k-1} V_{0}^{*} \otimes M_{l+1} \leftarrow \Lambda^{k} V_{0}^{*} \otimes M_{l} \leftarrow \Lambda^{k+1} V_{0}^{*} \otimes M_{l-1} \leftarrow \cdots,
$$

defined by the multiplication maps $M_{l+1} \leftarrow V_{0}^{*} \otimes M_{l}$ (see [Gr]). These Koszul complexes tensorized by $R(-k-l)$ fit as vertical lines into a double complex with $H_{5}$-equivariant differentials. The horizontal lines are obtained by tensoring $M_{l}$ with the Koszul complex resolving $\mathbb{C}(-l)$.

Following $[\mathrm{Po}]$ we first consider the case where $M$ is additionally invariant under $\iota$. In terms of $G_{5}$-modules the double complex reads:

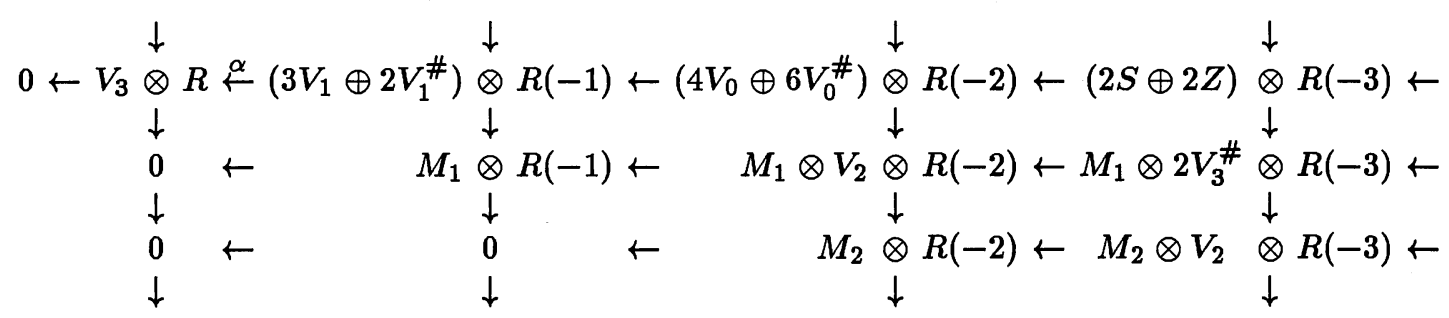

For every $k$ the induced maps $F_{k-1, l^{\prime}} \otimes R\left(-l^{\prime}\right) \leftarrow F_{k, l} \otimes R(-l)$ are just the components of $\alpha_{k}$. In order to study $\alpha_{1}$ we use the notations of the beginning of Section 4 . We want to compute the restriction of the differential $\alpha$ of the double complex to a 5 -dimensional $G_{5}$-invariant subspace of $V_{3} \otimes V_{0}^{*}$. Therefore we choose a decomposition of $V_{3} \otimes V_{0}^{*} \cong 3 V_{1} \oplus 2 V_{1}^{\#}$ into irreducible $G_{5}$-modules (the subspaces selected in Section 4 are not $\iota$-invariant). Namely, we consider the subspaces whose $i^{\text {th }}$ basis vector is given by $f_{i}^{(0)}, f_{i}^{(1)}+f_{i}^{(4)}, f_{i}^{(2)}+f_{i}^{(3)}$ and $f_{i}^{(2)}-f_{i}^{(3)}, f_{i}^{(1)}-f_{i}^{(4)}$ resp. Every $a=\left(a_{0}: \cdots: a_{4}\right) \in \mathbb{P}^{4}$ with either $a_{3}=a_{4}=0$ or $a_{0}=a_{1}=a_{2}=0$ defines a $G_{5}$-invariant subspace

$$
V^{a} \subset V_{3} \otimes V_{0}^{*} \cong 3 V_{1} \oplus 2 V_{1}^{\#}
$$

The $i^{\text {th }}$ basis vector of $V^{a}$ is the linear combination of the $i^{\text {th }}$ basis vectors above with $a_{0}, \ldots, a_{4}$ as coefficients. The restriction to $V^{a}$ of the differential $\alpha$ of the double complex is given by the Moore matrix

$$
M_{p}(y) \quad \text { with } \quad p=\left(a_{0}: a_{1}+a_{4}: a_{2}+a_{3}: a_{2}-a_{3}: a_{1}-a_{4}\right) \in \mathbb{P}^{4} .
$$

Then $p \in \mathbb{P}_{+}^{2}$ iff $V^{a} \cong V_{1}$ and $p \in \mathbb{P}_{-}^{1}$ iff $V^{a} \cong V_{1}^{\#}$.

Suppose for the moment that $M_{1}$ is 10 -dimensional. Then there are three possibilities for $M_{1}$, namely $M_{1} \cong 2 V_{1}^{\#}, M_{1} \cong V_{1} \oplus V_{1}^{\#}$ or $M_{1} \cong 2 V_{1}$. Correspondingly, there are the following three possibilities for the representing plane of $M$ :

(5.1), (i) $<p_{1}, p_{2}, p_{3}>=\mathbb{P}_{+}^{2}$.

(5.1), (ii) $<p_{1}, p_{2}>\subset \mathbb{P}_{+}^{2}, \quad p_{3} \in \mathbb{P}_{-}^{1}$.

(5.1), (iii) $p_{1}=\left(a_{0}: a_{1}: a_{2}: a_{2}: a_{1}\right) \in \mathbb{P}_{+}^{2}, \quad<p_{2}, p_{3}>=\mathbb{P}_{-}^{1}$.

(5.1), (i) gives the module of Section 3 corresponding to the HM-surfaces. By Proposition 4.2 we can choose $p_{1}, p_{2}$ and $p_{3}$ to be the non-trivial 2 -torsion points on any smooth elliptic 
curve $E_{(\lambda: \mu)}(x)$. Due to the results on the incidence scroll in Section 4 we will have some control over the module $M$ in the case where all three points are on a smooth $E_{(\lambda ; \mu)}(x)$. For example, the following is a special case of (5.1), (ii):

(5.1), (ii)' $<p_{1}, p_{2}>\subset \mathbb{P}_{+}^{2}$ is the line spanned by two non-trivial 2-torsion points of $E_{(\lambda: \mu)}(x)$ and $p_{3} \in \mathbb{P}_{-}^{1}$ is its origin.

Let us fix one smooth curve $E_{(\lambda ; \mu)}(x)$ and denote by $\tau_{i}, i=\overline{1,3}$ the non-trivial 2-torsion points and by $\rho$ one of the non-trivial 3-torsion points of this curve. The following cases, in which $M$ is not invariant under $\iota$, are of particular interest because of Lemma 4.18 and Remark 4.17:

(5.1), (iv) $p_{i}=\tau_{i}, i=1,2$ and $p_{3}=\tau_{3}+\rho$.

(5.1), (v) $p_{i}=\tau_{i}+\rho, i=\overline{1,3}$.

In the sequel we will analyse graded $R$-modules $M$ with a presentation matrix $\alpha_{1}$ defined by a representing plane $\Pi=<p_{1}, p_{2}, p_{3}>\subset \mathbb{P}^{4}(x)$ with particular interest in the cases of (5.1).

Let us first check under which conditions $M$ is artinian. We need the following notation. Let $N(u, y)$ be the $5 \times 5$-matrix with bilinear entries in $u$ 's and $y$ 's defined by the relation

$$
\left(u_{0}, u_{1}, u_{2}, u_{3}, u_{4}\right) \cdot M(x, y)=\left(x_{0}, x_{1}, x_{2}, x_{3}, x_{4}\right) \cdot N(u, y) .
$$

In other words,

$$
N(u, y)=\left(u_{2 i+j} y_{i+j}\right)_{i, j \in \mathbb{Z}_{\mathfrak{b}}} .
$$

Lemma 5.3. If $y \in \mathbb{P}^{4}(y)$ is in the support of the module $M$, then the matrix $M(x, y)$ has rank at most 4 on the plane $\Pi$ and there exists a point $u \in \mathbb{P}^{4}(u)$ such that $p_{i} \cdot N(u, y)=0$, $i=\overline{1,3}$. In particular, $M$ is artinian iff its representing plane $\Pi$ is not in the kernel of the linear map

$$
N(u, y): \mathbb{P}^{4}(x) \rightarrow \mathbb{P}^{4}(x), \quad x \mapsto x \cdot N(u, y)
$$

for every $(u, y) \in \mathbb{P}^{4}(u) \times \mathbb{P}^{4}(y)$.

Proof. If the module $M$ has support at $y$, then there exists a point $u$ defining a common left side syzygy for all the three Moore blocks of $\alpha_{1}$ at that point $y$. The lemma now follows from the defining relation (5.2).

Lemma 5.4. Let $y$ be in the support of the module $M$. Then we have the following two possibilities:

(i) If $y$ is not on a HM-line, then $\Pi$ is contained in the HM-quintic $\{\operatorname{det} M(x, y)=0\}$. In particular, the image of $\Pi$ under the rational map

$$
\Theta: \mathbb{P}^{4}(x) \rightarrow \mathbb{P}^{5^{*}} \quad x \mapsto\left(\gamma_{0}(x): \gamma_{1}(x): \cdots: \gamma_{5}(x)\right)
$$

does not span all of $\mathbb{P}^{5^{*}}$.

(ii) If $y$ is on one of the HM-lines, then the plane $\Pi$ contains two lines of a $G_{5}$-invariant pentagon. In particular, $\Pi$ does not intersect a smooth elliptic curve $E_{(\lambda: \mu)}(x)$ nor does it contain a HM-line.

Proof. (i) If $y$ is not on a HM-line then by Remark $4.1 \operatorname{det} M(x, y)$ is not vanishing identically and $\{\operatorname{det} M(x, y)=0\}$ is a HM-quintic which must contain $\Pi$ by Lemma 5.3. 
(ii) By $H_{5}$-invariance we may assume that $y \in \mathbb{P}_{-}^{1}$. Then $M(x, y)$ is skew-symmetric (up to column permutations), it has the left side syzygy

$$
I_{E_{y}}(x) \cdot M(x, y)=0,
$$

and it has rank 4 outside $E_{y}$ by Lemma 4.4. Lemma 5.3 and the relation (5.2) tell us that $I_{E_{y}}(x)$ is a multiple of a common $u$ for every $x \in \Pi$. The entries of $I_{E_{y}}(x)$ are the quadrics which cut out the elliptic curve $E_{y}$. These define a quadro-cubic Cremona transformation from $\mathbb{P}^{4}$ to $\mathbb{P}^{4}$ which by the above must contract the plane $\Pi$. There are no such planes if $E_{y}$ is smooth since the Cremona transformation contracts only $\operatorname{Sec} E$ which does not contain any plane (see [Seg], [Sem] and also [ADHPR] for the properties of the quadro-cubic Cremona transformation). If $E_{y}$ is a pentagon, then precisely the planes spanned by two lines of the pentagon are contracted. The second assertion in (ii) can be checked from the explicit equations of the elliptic curves and the HM-lines resp.

Proposition 5.5. The module $M$ defined by the plane $\Pi=<p_{1}, p_{2}, p_{3}>$ is artinian in the cases (i), (ii)', (iv) and (v) of (5.1) where all three points lie on a smooth $G_{5}$-invariant elliptic normal curve $E \subset \mathbb{P}^{4}$.

Proof. Suppose that the module $M$ has support at some point $y \in \mathbb{P}^{4}(y)$. Then $\Pi$ is contained in the HM-quintic $\{\operatorname{det} M(x, y)=0\}$ by Lemma 5.4, (ii) and (i). We will show that this quintic coincides with $\operatorname{Sec} E$, a contradiction, since $\operatorname{Sec} E$ contains no planes.

Let more generally $U$ be any HM-quintic containing all three lines $l_{i j}=\overline{p_{i} p_{j}}, i<j$. The $l_{i j}$ are secant lines to $E$, and we denote by $T_{i j}$ the translation scrolls of $E$ generated by these lines (compare [BHM2]). $T_{i j}$ has degree 5 if $p_{i}-p_{j}$ is 2-torsion and degree 10 otherwise. For every $i<j$ there exists precisely one section $s_{i j}$ of the HM-bundle $\mathcal{E}(3)$ whose reduced zeroscheme is $T_{i j}$ [BHM2]. For our purpose it suffices to show that $U$ contains at least two different $T_{i j}$ 's since then $U$ is uniquely determined as the wedge product of the corresponding sections of the HM-bundle. In particular, $U$ must coincide with $\operatorname{Sec} E$, because this HMquintic contains all translation scrolls of $E$ as above.

The orbit of $l_{i j}$ under $G_{5}$ consists of 25 lines if $l_{i j}$ is $\iota$-invariant, i.e., if $p_{i}+p_{j}=0$ or $p_{i}$ and $p_{j}$ are 2-torsion, and of 50 lines otherwise. By Bezout $U$ contains $E$ and thus $T_{i j}$ unless possibly if $l_{i j}$ is $\iota$-invariant and $T_{i j}$ has degree 10 . In the cases (i) and (ii)' $p_{1}, p_{2}$ and $p_{3}$ are 2-torsion points. Therefore the $T_{i j}$ are the three distinct quintic elliptic scrolls which are the 2-torsion translation scrolls of $E$ and they are all contained in $U$. In case (iv) $T_{12}$ has degree 5 , whereas $T_{13}$ and $T_{23}$ are two different scrolls of degree 10 , and the lines $l_{13}$ and $l_{23}$ are not $\iota$-invariant. In case $(\mathrm{v})$ non of the lines is $\iota$-invariant and the three scrolls of degree 10 are different. In any case $U$ contains three different $T_{i j}$ 's.

Proposition 5.6. Let $p_{1}=\left(a_{0}: a_{1}: a_{2}: a_{2}: a_{1}\right) \in \mathbb{P}_{+}^{2}, p_{2}=(0: 1: 0: 0:-1)$ and $p_{3}=(0: 0: 1:-1: 0)$ as in case (5.1), (iii). Then the module $M$ defined by the plane $\Pi=<p_{1}, p_{2}, p_{3}>$ is artinian iff $p_{1} \in \mathbb{P}_{+}^{2} \backslash \Delta$, where $\Delta$ is the union of the 6 lines

$$
x_{0}=0 \quad \text { and } \quad x_{0}+\xi^{i} x_{1}+\xi^{-i} x_{2}=0, \quad i \in \mathbb{Z}_{5} \text {, }
$$

corresponding to the unique minimal $A_{5}$-orbit in $\left(\mathbb{P}_{+}^{2}\right)^{*}$.

Proof. By Lemma 5.3 we have to show that $\Pi$ is contained in the kernel of the linear map

$$
N(u, y): \mathbb{P}^{4}(x) \rightarrow \mathbb{P}^{4}(x), \quad x \mapsto x \cdot N(u, y)
$$


for some $(u, y) \in \mathbb{P}^{4}(u) \times \mathbb{P}^{4}(y)$ iff $p_{1} \in \Delta$. The variety

$$
I_{-}=\left\{(u, y) \in \mathbb{P}^{4}(u) \times \mathbb{P}^{4}(y) \mid \mathbb{P}_{-}^{1} \subset \operatorname{ker} N(u, y)\right\}
$$

is defined by the 10 bilinear forms

$$
u_{i+2} y_{i+1}-u_{i+3} y_{i+4}=u_{i+4} y_{i+2}-u_{i+1} y_{i+3}=0, \quad i \in \mathbb{Z}_{5}
$$

The saturation of these forms with respect to $y_{0}, \ldots, y_{4}$ contains the 10 cubic equations

$$
v^{+}(u)=v^{-}(u)=0
$$

which cut out the 30 vertices of the 6 complete pentagons in $S_{15} \subset \mathbb{P}^{4}(u)$ (compare Remark 4.9 , (i)). Conversely, every such vertex is contained in the image of $I_{-}$under the projection to $\mathbb{P}^{4}(u)$. Under the action of the normalizer $N_{5}$ of the Heisenberg group these pentagons are equivalent to the one with vertices $e_{0}, \ldots, e_{4}$. For $\left(u=e_{i}, y\right) \in I_{-}$the plane $\Pi$ is contained in $\operatorname{ker} N(u, y)$ iff $p_{1}$ is on the line $x_{0}=0$. The lemma follows by the action of $N_{5}$.

From now on we will always suppose in case (5.1), (iii) that $p_{1} \in \mathbb{P}_{+}^{2} \backslash \Delta$.

Next we will study the first order linear syzygies of $\alpha_{1}$. It is clear from the representation theory described at the beginning of Section 4 and from the double complex of the Koszul cohomology that these come in blocks of $L$-matrices. We recall from Section 4 that the product matrices $M_{p}(y) L_{q}(y)$ have quadratic entries in $y$ 's with coefficients that are bilinear forms in $x$ 's and $z$ 's defining a morphism

$$
\Phi_{T}: \mathbb{P}^{4}(x) \times \mathbb{P}^{4}(z) \rightarrow \mathbb{P}^{14}
$$

which is a projection of the Segre embedding. Five first order linear syzygies of $\alpha_{1}$ are given by a non-trivial relation

$$
M_{p_{1}}(y) L_{q_{1}}(y)+M_{p_{2}}(y) L_{q_{2}}(y)+M_{p_{3}}(y) L_{q_{3}}(y)=0
$$

with $q_{i} \in \mathbb{P}^{4}(z)$ or $L_{q_{i}}(y)=0, i=\overline{1,3}$. We will express such a relation in terms of $\Phi_{T}$ and thus in terms of the three linear subspaces defined as $R_{p_{i}}=\Phi_{T}\left(\left\{p_{i}\right\} \times \mathbb{P}^{4}(z)\right), i=\overline{1,3}$ (clearly, these subspaces are $\mathbb{P}^{4}$ 's). First notice that the following case cannot occur, since $\Phi_{T}$ is a morphism:

(I) $\left(p_{i}, q_{i}\right)$ is a base point of $\Phi_{T}$ for one $i$, while the other two $L$-matrices are zero.

Therefore every five first order linear syzygies of $\alpha_{1}$ are a linear combination of those corresponding to one of the following cases.

(II) $\Phi_{T}\left(p_{i}, q_{i}\right)=\Phi_{T}\left(p_{j}, q_{j}\right)$ is a double point of $R_{p_{i}} \cup R_{p_{j}}$ for one pair $i<j$, while the third $L$-matrix is zero.

(III) The three different points $\Phi_{T}\left(p_{i}, q_{i}\right)$ are collinear, i.e., these points define a proper trisecant to $R_{p_{1}} \cup R_{p_{2}} \cup R_{p_{3}}$. 
Lemma 5.7. Let $c \in\{1,2\}$ and suppose that $R_{p_{1}}, R_{p_{2}}$ and $R_{p_{3}}$ span a $\mathbb{P}^{14-c}$. Then $\alpha_{1}$ has precisely $5 c$ first order linear syzygies.

Proof. (i) If $R_{p_{1}}, R_{p_{2}}$ and $R_{p_{3}}$ span a $\mathbb{P}^{13}$, then there are two cases:

a) Two of the $\mathbb{P}^{4}$ 's meet in a point and their span is disjoint from the third one.

b) The $\mathbb{P}^{4}$ 's are pairwise disjoint and any of the three intersects the span of the two others in a point.

In case a) there is no proper trisecant. In case b) let $\{i, j, k\}=\{1,2,3\}$. We spot one trisecant by projecting from the point of intersection of $R_{p_{k}}$ with the span of $R_{p_{i}}$ and $R_{p_{j}}$ to a $\mathbb{P}^{8}$, where the images of $R_{p_{i}}$ and $R_{p_{j}}$ have to meet in a point. This trisecant is the unique proper trisecant (independent of the choice of $i, j, k$ ). So in both cases there are precisely 5 first order linear syzygies.

(ii) If $R_{p_{1}}, R_{p_{2}}$ and $R_{p_{3}}$ span a $\mathbb{P}^{12}$, then there are five cases:

a) Two of the $\mathbb{P}^{4}$ 's meet in a line and their span is disjoint from the third one.

b) One of the $\mathbb{P}^{4}$ 's meets any of the others in a point, the two others do not meet.

c) $R_{p_{1}}, R_{p_{2}}$ and $R_{p_{3}}$ meet in a common point.

d) Two of the $\mathbb{P}^{4}$ 's meet in a point and their span intersects the third one in another point.

e) The $\mathbb{P}^{4}$ 's are pairwise disjoint and any of the three intersects the span of the two others in a line.

Arguing as above we see that there is no proper trisecant in the cases a), b) and c), one in case d) and a pencil of them in case e). Altogether, there are precisely 10 first order linear syzygies in each case.

Proposition 5.8. For generic choices in (5.1), (ii) $\alpha_{1}$ has precisely 5 first order linear syzygies. In the other cases of (5.1) there are precisely 10 such syzygies $\left(L_{q_{i j}}\right)_{\substack{1 \leq i \leq 3 \\ 1 \leq j \leq 2}}$. The points $q_{i j}$ can be chosen as follows:

(i) $L_{q_{31}}=L_{q_{22}}=0, \quad q_{i j} \in \mathbb{P}_{-}^{1}$ otherwise, $q_{11}=q_{12}$.

(ii)' $L_{q_{31}}=L_{q_{22}}=0, q_{11}, q_{12}, q_{21} \in \mathbb{P}_{-}^{1}, q_{11}=q_{12}$ and $q_{32}=\psi_{5}\left(o_{E_{(\lambda: \mu)}}\right) \in \mathbb{P}_{+}^{2}$.

(iii) $q_{11}=(0: 1: 0: 0:-1), q_{21}=\left(0:-a_{1}: a_{0}: a_{0}:-a_{1}\right), q_{31}=\left(-2 a_{1}: a_{2}: 0: 0: a_{2}\right)$, $q_{12}=(0: 0: 1:-1: 0), q_{22}=\left(2 a_{2}: 0:-a_{1}:-a_{1}: 0\right)$ and $q_{32}=\left(0: a_{0}:-a_{2}:-a_{2}: a_{0}\right)$.

(iv) $L_{q_{31}}=0, q_{i 1} \in \mathbb{P}_{-}^{1}, i=1,2$ and $q_{32} \in E_{(\lambda: \mu)}\left(\tau_{3}\right)$ not on a HM-line.

(v) $L_{q_{31}}=L_{q_{22}}=0$, and $q_{i j} \in E_{(\lambda: \mu)}^{\prime}$ not on a HM-line otherwise, $q_{11}=q_{12}$.

Proof. We first study the situation, where all three points $p_{1}, p_{2}$ and $p_{3}$ are on the elliptic curve $E_{(\lambda: \mu)}(x)$. Let $\mathbb{P}_{(\lambda: \mu)}^{4} \subset \mathbb{P}^{14}$ denote the $\mathbb{P}^{4}$ of quadrics defining $E_{(\lambda: \mu)}$. Then $\mathbb{P}_{(\lambda: \mu)}^{4}$ meets $W:=\Phi_{T}\left(E_{(\lambda ; \mu)} \times \mathbb{P}^{4}(z)\right)$ along the degree 15 scroll $S_{(\lambda: \mu)}$ as in Lemma 4.15. Now define $W^{\prime}$ as the $\mathbb{P}^{2}$-scroll obtained by projecting $W$ from $\mathbb{P}_{(\lambda: \mu)}^{4}$.

Lemma 5.9. (i) $W^{\prime}$ is a linearly normal, smooth $\mathbb{P}^{2}$-scroll in $\mathbb{P}^{9}$ of degree 10.

(ii) $W^{\prime}$ has no proper trisecants.

Proof. Recall from Proposition 4.16 that $S_{(\lambda: \mu)}$ is a birational image of the symmetric product $S^{2}\left(E_{(\lambda: \mu)}\right)$. More precisely, if $\mathcal{E}_{(\lambda: \mu)}$ denotes the unique non-split extension

$$
0 \rightarrow \mathcal{O}_{E_{(\lambda: \mu)}}\left(-o_{E_{(\lambda: \mu)}}\right) \rightarrow \mathcal{E}_{(\lambda: \mu)} \rightarrow \mathcal{O}_{E_{(\lambda: \mu)}} \rightarrow 0
$$

where $o_{E_{(\lambda: \mu)}}$ is the origin of $E_{(\lambda: \mu)}$, then the correspondence in Proposition 4.16 maps $\mathbb{P}\left(\mathcal{E}_{(\lambda: \mu)}\left(-7 o_{E_{(\lambda ; \mu)}}\right)\right)$ to $S_{(\lambda ; \mu)}$ via the morphism induced by $\left.\left.\mathcal{O}_{\mathbb{P}\left(\mathcal{E}_{(\lambda ; \mu)}\left(-7 o_{E}(\lambda ; \mu)\right.\right.}\right)\right)$. Then 
the identification in Lemma 4.15 of $S_{(\lambda: \mu)}$ with the image of $I_{(\lambda: \mu)}$ under $\Phi_{T}$ accounts for the exact sequence of vector bundles on $E_{(\lambda: \mu)}$ :

$$
0 \rightarrow \mathcal{E}_{(\lambda: \mu)}\left(-7 o_{E_{(\lambda ; \mu)}}\right) \rightarrow H^{0}\left(\mathcal{J}_{E_{(\lambda ; \mu)}}(2)\right) \otimes \mathcal{O}_{E_{(\lambda ; \mu)}}(-1) \rightarrow Q \rightarrow 0
$$

where $Q$ is a rank 3 vector bundle such that $W^{\prime}$ coincides with the image of $\mathbb{P}(\mathcal{Q})$ under the map induced by $\mathcal{O}_{\mathbb{P}(\Omega)}(1)$. Twisting this sequence by $\mathcal{O}_{E_{(\lambda: \mu)}}(1)$ we get

$$
0 \rightarrow \mathcal{E}_{(\lambda: \mu)}\left(-2 o_{E_{(\lambda: \mu)}}\right) \rightarrow H^{0}\left(\mathcal{J}_{E_{(\lambda ; \mu)}}(2)\right) \otimes \mathcal{O}_{E_{(\lambda ; \mu)}} \rightarrow \mathcal{Q}(1) \rightarrow 0
$$

Together with its dual this sequence presents naturally $\mathbb{P}\left(\mathcal{Q}^{\vee}(-1)\right) \subset\left(\mathbb{P}^{4}\right)^{*} \times E_{(\lambda: \mu)}$ as the dual of the scroll $\mathbb{P}\left(\mathcal{E}_{(\lambda: \mu)}\left(-2 o_{E_{(\lambda: \mu)}}\right)\right) \subset \mathbb{P}^{4} \times E_{(\lambda: \mu)}$. The projections to $\mathbb{P}^{4}$ and its dual resp. map the $\mathbb{P}^{1}$-bundle to an elliptic quintic scroll and the $\mathbb{P}^{2}$-bundle to the dual quintic trisecant scroll resp. Thus we know from [ADHPR] that $\mathcal{Q}\left(3 o_{\left.E_{(\lambda ; \mu)}\right)}\right)$ is indecomposable of degree -1 . Furthermore $Q^{\vee}\left(-3 o_{E_{(\lambda: \mu)}}\right)$ has one section. In fact, $h^{0}\left(Q^{\vee} \otimes \mathcal{L}^{\vee}\right)=1$ for all degree 3 line bundles $\mathcal{L}$ on $E_{(\lambda: \mu)}$ and $h^{0}\left(\mathcal{Q}^{\vee}\right)=10$. Thus $W^{\prime}$ is linearly normal of degree 10 in $\mathbb{P}^{9}$, and every triple of planes in $W^{\prime}$ spans a hyperplane. Therefore any two planes must span a $\mathbb{P}^{5}$ and no three planes can have a trisecant. We conclude that $W^{\prime}$ is smooth with no trisecant.

As a consequence of the lemma we see that $W$ is smooth outside $\mathbb{P}_{(\lambda: \mu)}^{4}$ and that any proper trisecant to $W$ lies in $\mathbb{P}_{(\lambda: \mu)}^{4}$, and thus must be a trisecant to $S_{(\lambda: \mu)}$. In particular, up to linear combinations, 5 first order linear syzygies of $\alpha_{1}$ correspond either to a point of intersection of two of the three rulings $\tilde{R}_{p_{i}}=R_{p_{i}} \cap \mathbb{P}_{(\lambda: \mu)}^{4}$, or to a proper trisecant of these rulings.

In the cases (i) and (ii)' $\tilde{R}_{p_{1}} \cap \tilde{R}_{p_{2}} \cap \tilde{R}_{p_{3}}=\left\{o_{E_{(\lambda ; \mu)}}\right\}$, while in case (v) the rulings intersect in the chosen 3-torsion point $\rho$ (compare Proposition 4.16, (iii) and Remark 4.17, (ii)). Here we identify as always points on $E_{(\lambda: \mu)}(x)$ with points on $E_{(\lambda: \mu)}(w)$. In case (iv), however, $\tilde{R}_{p_{3}}$ hits the plane spanned by $\tilde{R}_{p_{1}}$ and $\tilde{R}_{p_{2}}$ in the point $\Phi_{T}\left(p_{3}, q_{32}\right)$, where $q_{32} \in E_{(\lambda: \mu)}\left(\tau_{3}\right)$ by Lemma 4.18 and Corollary 4.21 . Notice more precisely that $\Phi_{T}\left(p_{3}, q_{32}\right)$ lies on a ruling of $S_{(\lambda: \mu)}$ which meets $E_{(\lambda: \mu)}$ in a non-trivial 6-torsion point. In both cases (iv) and (v) the points $q$ are not on a HM-line, since any point $(p, q)$ with $q$ in $S^{2}\left(E_{(\lambda: \mu)}\right)$ on a HM-line would map to a ruling of $S_{(\lambda: \mu)}$ which meets $E_{(\lambda: \mu)}$ in a non-trivial 5-torsion point again by Corollary 4.21. Therefore there are precisely 10 first order linear syzygies in all cases where $p_{1}, p_{2}$ and $p_{3}$ lie on the elliptic curve $E_{(\lambda: \mu)}(x)$, and the points $q$ can be chosen as claimed.

Of course (i) is known from [De2], [Ma3] and [Ma4] and can also be seen by just computing the equations of $R_{p_{1}}, R_{p_{2}}$ and $R_{p_{3}}$, where, say, $p_{1}=(1: 0: 0: 0: 0), p_{2}=(0: 1: 0: 0: 1)$ and $p_{3}=(0: 0: 1: 1: 0)$. A similar calculation, depending on $p_{1}=\left(a_{0}: a_{1}: a_{2}: a_{2}: a_{1}\right) \epsilon$ $\mathbb{P}_{+}^{2} \backslash \Delta$, and with $p_{2}=(0,1,0,0,-1)$ and $p_{3}=(0,0,1,-1,0)$, shows the existence of precisely ten first order linear syzygies in case (iii). It is easy to compute that $\sum_{i=1}^{3} M_{p_{i}} \cdot L_{q_{i j}}=0$, $j=1,2$, when the $q_{i j}$ are chosen as claimed (see also [Po]).

We finally need to show that for generic choices in (5.1), (ii) $\alpha_{1}$ has precisely 5 first order linear syzygies. For this, we consider first $S_{-}=\Phi_{T}\left(\mathbb{P}_{-}^{1}(x) \times \mathbb{P}_{+}^{2}(z)\right)$ and $S_{+}=\Phi_{T}\left(\mathbb{P}_{+}^{2}(x) \times\right.$ $\left.\mathbb{P}_{-}^{1}(z)\right)$. Then $S_{-}$is contained in the $\mathbb{P}^{5} \subset \mathbb{P}^{14}(t)$ defined by

$$
t_{00}=t_{10}=t_{20}=t_{02}+t_{03}=t_{12}+t_{13}=t_{22}+t_{23}=t_{01}+t_{04}=t_{11}+t_{14}=t_{21}+t_{24}=0,
$$


while $S_{+}$is contained in the $\mathbb{P}^{3}$ inside this $\mathbb{P}^{5}$ cut out by the additional

$$
t_{11}+t_{03}=t_{01}+t_{22}=0 .
$$

Thus for $p_{1}, p_{2} \in \mathbb{P}_{+}^{2}$ and $p_{3} \in \mathbb{P}_{-}^{1}$ the subspaces $R_{p_{1}}$ and $R_{p_{2}}$ will meet $S_{+}$in a line while $R_{p_{3}}$ will meet $S_{-}$in a plane. Since this latter plane will meet the $\mathbb{P}^{3}$ of $S_{+}$in at least a point there must be at least one not necessarily proper trisecant line for the $R_{p_{i}}, i=\overline{1,3}$. Thus the corresponding $\alpha_{1}$ has at least 5 linear syzygies. It now suffices to find three points such that the corresponding $\mathbb{P}^{4}$ 's span a $\mathbb{P}^{13}$. E.g., we may take $p_{1}=(1: 0: 0: 0: 0)$, $p_{2}=(0: 1: 0: 0: 1)$ and $p_{3}=(0: 1: 1:-1:-1)$ in which case $R_{p_{1}}, R_{p_{2}}$ and $R_{p_{3}}$ span the hyperplane defined by

$$
2 t_{20}+t_{11}-t_{02}-t_{03}+t_{14}=0
$$

in $\mathbb{P}^{14}(t)$.

Note that in the $G_{5}$-invariant cases the first order linear syzygies are decomposed into $G_{5}$ representations. This will be the case also for the rest of the syzygies below, although we do not make it explicit since $H_{5}$-invariance is enough to make the computations.

Proposition 5.10. In the above cases of (5.1) with 10 first order linear syzygies $\left(L_{q_{i j}}\right)$ the linear part of the minimal free resolution of $M$, written in terms of $\mathrm{H}_{5}$-modules, is as follows:

$$
V_{3} \otimes R \stackrel{\left(M_{p_{i}}\right)}{\leftarrow} 3 V_{1} \otimes R(-1) \stackrel{\left(L_{q_{i j}}\right)}{\leftarrow} 2 V_{0} \otimes R(-2) \stackrel{K}{\leftarrow} a I \otimes R(-3) \leftarrow 0,
$$

where $K$ is a direct sum of Koszul maps $[y]^{t}=\left(y_{0}, \ldots, y_{4}\right)^{t}$. Moreover $a=2$ in the case (i), $a=1$ in the cases (ii)' and (iv) and $a=0$ in the cases (iii) and (v).

Proof. We will take into account the results of Proposition 5.8 concerning the points $q_{i j}$. Notice that $L_{q}(y)[y]^{t}=0$ iff $q \in \mathbb{P}_{-}^{1}$. In this case $[y]^{t}$ defines the only linear syzygy of $L_{q}(y)$ (compare Remark 4.6). If however $q$ is a point not on one of the HM-lines, then $\operatorname{det} L_{q}(y)$ does not vanish identically by Remark 4.1 , and $L_{q}(y)$ has no syzygies at all. This settles the cases (i), (ii)', (iv) and (v). In case (iii) we compute that the linear syzygies of $\left(L_{q_{11}}, L_{q_{12}}\right)$ are precisely a direct sum of two copies of $[y]^{t}$. These do not give rise to a linear syzygy of $\left(L_{q_{i j}}\right)_{\substack{2 \leq i \leq 3 \\ 1 \leq j \leq 2}}$.

Proposition 5.11. In the above cases of (5.1) with 10 first order linear syzygies $\left(L_{q_{i j}}\right)$ there are 4 first order quadratic syzygies of $\alpha_{1}$ in the case (i), 2 in the case (iii) and 1 in the cases (ii)', (iv) and (v).

Proof. The decomposition of the space of quadrics in $\mathbb{P}^{4}$ into irreducible $H_{5}$-modules is $S^{2} V_{0}^{*} \cong 3 V_{3}$. Explicitely such a decomposition is given by distinguishing the following subspaces:

$$
<y_{i}^{2}>_{i \in \mathbb{Z}_{5}}, \quad<y_{i+1} y_{i+4}>_{i \in \mathbb{Z}_{5}}, \quad<y_{i+2} y_{i+3}>_{i \in \mathbb{Z}_{5}} .
$$

Moreover, $S^{2} V_{0}^{*} \otimes V_{1}$ decomposes into characters of $H_{5}$. ¿From the double complex of the Koszul cohomology we see that quadratic syzygies of $\alpha_{1}$ are elements in

$$
\operatorname{Hom}_{H_{5}}\left(\chi \otimes \mathcal{O}(-3), 3 V_{1} \otimes \mathcal{O}(-1)\right) \cong \operatorname{Hom}_{H_{5}}\left(\chi, S^{2} V_{0}^{*} \otimes 3 V_{1}\right),
$$

where $\chi$ is some character of $H_{5}$. 
We first study those first order quadratic syzygies which involve the trivial character. These come in blocks of column vectors of type

$$
L_{b}^{\prime}(y)=\left(b_{0} y_{i}^{2}+b_{1} y_{i+2} y_{i+3}+b_{2} y_{i+1} y_{i+4}\right)_{i \in \mathbb{Z}_{5}},
$$

where $b=\left(b_{0}: b_{1}: b_{2}\right)$ is a parameter point in $\mathbb{P}^{2}$. The $i^{\text {th }}$ entry of the $5 \times 1$-matrix $M_{x}(y) L_{b}^{\prime}(y)$ is a cubic in the span of

$$
y_{i}^{3}, y_{i} y_{i+1} y_{i+4}, y_{i} y_{i+2} y_{i+3}, y_{i+1}^{2} y_{i+3}, y_{i+1} y_{i+2}^{2}, y_{i+2} y_{i+4}^{2}, y_{i+3}^{2} y_{i+4}
$$

$i=\overline{0,4}$, whereas the coefficients are bilinear forms in $x$ 's and $b$ 's. We collect these forms in the column vector

$$
T^{\prime}(x, b)=\left(\begin{array}{c}
x_{0} b_{0} \\
x_{0} b_{2}+\left(x_{1}+x_{4}\right) b_{1} \\
x_{0} b_{1}+\left(x_{2}+x_{3}\right) b_{2} \\
x_{2} b_{0}+x_{4} b_{2} \\
x_{3} b_{1}+x_{4} b_{0} \\
x_{1} b_{2}+x_{3} b_{0} \\
x_{1} b_{0}+x_{2} b_{1}
\end{array}\right)
$$

$T^{\prime}$ defines a rational map

$$
\Phi_{T^{\prime}}: \mathbb{P}^{4}(x) \times \mathbb{P}^{2}(b)--\rightarrow \mathbb{P}^{6}(s),
$$

well-defined outside the codimension 5 set

$$
B_{\Phi_{T^{\prime}}}=\left(\mathbb{P}_{-}^{1}(x) \times \mathbb{P}^{2}(b)\right) \cap\left\{b_{0}^{2}+b_{1} b_{2}=x_{2} b_{0}-x_{1} b_{2}=x_{2} b_{1}+x_{1} b_{0}=0\right\},
$$

which is a rational curve projecting bijectively onto $\mathbb{P}_{-}^{1}$ in the first factor, and onto the conic $\left\{b_{0}^{2}+b_{1} b_{2}=0\right\} \subset \mathbb{P}^{2}(b)$ in the second factor.

So a first order quadratic syzygy for $\alpha_{1}$ involving the trivial character is given by a non-trivial relation

$$
M_{p_{1}}(y) L_{r_{1}}^{\prime}(y)+M_{p_{2}}(y) L_{r_{2}}^{\prime}(y)+M_{p_{3}}(y) L_{r_{3}}^{\prime}(y)=0
$$

with $r_{i} \in \mathbb{P}^{2}(b)$ or $L_{q_{i}}^{\prime}(y)=0, i=\overline{1,3}$. We may express such a relation in terms of $\Phi_{T^{\prime}}$ and thus in terms of the three linear subspaces $S_{p_{i}}:=\Phi_{T^{\prime}}\left(\left\{p_{i}\right\} \times \mathbb{P}^{2}(b)\right) \subset \mathbb{P}^{6}(s), i=\overline{1,3}$. Then we see that every quadratic syzygy is a linear combination of those corresponding to one of the following cases.

(I) A basepoint, i.e., $\left(p_{i}, r_{i}\right) \in B_{\Phi_{T^{\prime}}}$ for some $i$, while the other two $L^{\prime}$-matrices are zero.

(II) A double point of $S_{p_{i}} \cup S_{p_{j}}$, i.e., $\Phi_{T^{\prime}}\left(p_{i}, r_{i}\right)=\Phi_{T^{\prime}}\left(p_{j}, r_{j}\right)$ for one pair $i<j$, while the third $L^{\prime}$-matrix is zero.

(III) A proper trisecant line of $S_{p_{1}} \cup S_{p_{2}} \cup S_{p_{3}}$, i.e., the three different points $\Phi_{T^{\prime}}\left(p_{1}, r_{1}\right)$, $\Phi_{T^{\prime}}\left(p_{2}, r_{2}\right)$ and $\Phi_{T^{\prime}}\left(p_{3}, r_{3}\right)$ are collinear.

The genuine quadratic syzygies which appear in the minimal resolution of the module $M$ are determined only modulo those quadratic syzygies which depend on the linear ones. These in turn appear as non-trivial relations

$$
\left(M_{p_{1}}(y) L_{q_{1}}(y)+M_{p_{2}}(y) L_{q_{2}}(y)+M_{p_{3}}(y) L_{q_{3}}(y)\right)\left(y_{0}, \ldots, y_{4}\right)^{t}=0
$$


Each summand is a product matrix $M_{p}(y) L_{q}(y)\left(y_{0}, \ldots, y_{4}\right)^{t}$ with cubic entries as above, but this time the coefficient vector is

$$
T^{\prime \prime}(x, z)=\left(\begin{array}{c}
x_{0} z_{0} \\
x_{0}\left(z_{1}+z_{4}\right)+\left(x_{1}+x_{4}\right)\left(z_{2}+z_{3}\right) \\
x_{0}\left(z_{2}+z_{3}\right)+\left(x_{2}+x_{3}\right)\left(z_{1}+z_{4}\right) \\
x_{2} z_{0}+x_{4}\left(z_{1}+z_{4}\right) \\
x_{3}\left(z_{2}+z_{3}\right)+x_{4} z_{0} \\
x_{1}\left(z_{1}+z_{4}\right)+x_{3} z_{0} \\
x_{1} z_{0}+x_{2}\left(z_{2}+z_{3}\right)
\end{array}\right) .
$$

$T^{\prime \prime}$ defines a rational map

$$
\Phi_{T^{\prime \prime}}: \mathbb{P}^{4}(x) \times \mathbb{P}^{4}(z)^{--\rightarrow} \mathbb{P}^{6}(s),
$$

which is a projection of $\Phi_{T}$. Therefore the linear map $\rho$ defined by

$$
b_{0} \mapsto z_{0}, \quad b_{1} \mapsto\left(z_{2}+z_{3}\right), \quad b_{2} \mapsto\left(z_{1}+z_{4}\right)
$$

gives rise to a commutative diagram

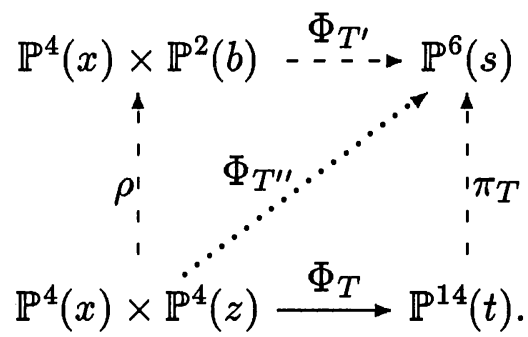

The projection $\pi_{T}$ restricted to $\Phi_{T}\left(\mathbb{P}^{4}(x) \times \mathbb{P}^{4}(z)\right)$ is well-defined outside the image via $\Phi_{T}$ of the base locus $B_{\Phi_{T^{\prime \prime}}}$ of $\Phi_{T^{\prime \prime}}$. The first component of $B_{\Phi_{T^{\prime \prime}}}$ is precisely the base locus $\mathbb{P}^{4}(x) \times \mathbb{P}_{-}^{1}(z)$ of the map $\rho: \mathbb{P}^{4}(x) \times \mathbb{P}^{4}(z)--\rightarrow \mathbb{P}^{4}(x) \times \mathbb{P}^{2}(b)$, while the second component

$\left(\left(\mathbb{P}_{-}^{1}(x) \times \mathbb{P}^{4}(z)\right) \cap\left\{z_{0}^{2}+\left(z_{1}+z_{4}\right)\left(z_{2}+z_{3}\right)=x_{2} z_{0}-x_{1}\left(z_{1}+z_{4}\right)=x_{2}\left(z_{2}+z_{3}\right)+x_{1} z_{0}=0\right\}\right.$

is a $\mathbb{P}^{2}$-bundle over $\mathbb{P}_{-}^{1}(x)$ which maps onto the quadric cone $\Gamma=\left\{z_{0}^{2}+\left(z_{1}+z_{4}\right)\left(z_{2}+z_{3}\right)=\right.$ $0\} \subset \mathbb{P}^{4}(z)$. Under this projection the plane over a point $o \in \mathbb{P}_{-}^{1}(x)$ is mapped to the plane on $\Gamma$ over the point $\psi_{5}(o)$ on $C_{+} \subset \mathbb{P}_{+}^{2}(z)$.

We will need one further fact, namely, that $S_{p}=\Phi_{T^{\prime}}\left(\{p\} \times \mathbb{P}^{2}(b)\right) \subset \mathbb{P}^{6}(s)$ coincides with the fixed line

$$
S_{-}=\left\{s_{0}=s_{1}=s_{2}=s_{3}+s_{5}=s_{4}+s_{6}=0\right\} \subset \mathbb{P}^{6}(s)
$$

for all $p \in \mathbb{P}_{-}^{1}(x)$, whereas $S_{p}$ is a plane inside the 4-dimensional linear subspace

$$
S_{+}=\left\{s_{3}-s_{5}=s_{4}-s_{6}=0\right\} \subset \mathbb{P}^{6}(s)
$$

for all $p \in \mathbb{P}_{+}^{2}$.

Now we may proceed to study the cases of (5.1), in each case determining all quadratic syzygies and comparing with Proposition 5.8 to see which of them are dependent on the linear ones. 
In case (i) we get three planes $S_{p}$ inside $S_{+}$. Each pair of planes intersect in a point inducing thus three quadratic syzygies of type (II), while there is exactly one proper trisecant to all three planes giving rise to a fourth syzygy of type (III). In this case the points giving linear syzygies are base points for the map $\rho$, so $\alpha_{1}$ has 4 genuine quadratic syzygies.

In case (ii)' the planes $S_{p_{1}}$ and $S_{p_{2}}$ span $S_{+}$while $S_{p_{3}}=S_{-}$. Since $S_{+}$and $S_{-}$span $\mathbb{P}^{6}(s)$, there are 2 candidates for quadratic syzygies: one of type (II) from the double point of the first two planes, and one of type (I) from the basepoint of $\Phi_{T^{\prime}}$ over the point $p_{3}$. Of the linear syzygies in this case, the points $\left(p_{1}, q_{11}\right),\left(p_{1}, q_{12}\right),\left(p_{2}, q_{21}\right)$ lie in the base locus $\left(\mathbb{P}^{4}(x) \times \mathbb{P}_{-}^{1}(z)\right)$ of $\rho$, while $\left(p_{3}, q_{32}\right)$ is mapped to the basepoint of $\Phi_{T^{\prime}}$ over $p_{3}$. Thus only the quadratic syzygy of type (I) is dependent on the linear ones, and $\alpha_{1}$ has exactly one genuine quadratic syzygy, which we may choose to be of type (II).

In case (iii) $S_{p_{1}}$ is a plane in $S_{+}$, while $S_{p_{2}}=S_{p_{3}}=S_{-}$. Thus there are 2 quadratic syzygies of type (I) from the basepoints over $p_{2}$ and $p_{3}$ and 2 quadratic syzygies of type (II) from the doublepoints on $S_{-}$. Of the linear syzygies, the points $\left(p_{1}, q_{11}\right)$ and $\left(p_{1}, q_{21}\right)$ are basepoints of $\rho$, while the other points are not. Therefore the linear syzygies give rise to 2 quadratic syzygies of type (I) or (II), depending on the choice of $p_{1}=\left(a_{0}: a_{1}: a_{2}: a_{2}: a_{1}\right) \in \mathbb{P}_{+}^{2} \backslash \Delta$. In any case, $\alpha_{1}$ has precisely 2 genuine quadratic syzygies.

For the last two cases we will take a closer look at the condition that all three points $p_{1}$, $p_{2}$ and $p_{3}$ lie on a smooth elliptic curve $E_{(\lambda: \mu)}(x)$. First we look at the projection via $\pi_{T}$ of the $\mathbb{P}^{4}$ - bundle $W=\Phi_{T}\left(E_{(\lambda: \mu)}(x) \times \mathbb{P}^{4}(z)\right) \subset \mathbb{P}^{14}(t)$ into $\mathbb{P}^{6}(s)$. Inside $W$ lies the image $\tilde{U}$ of $E_{(\lambda: \mu)}(x) \times \mathbb{P}_{+}^{2}(z)$. Since $\Phi_{T}\left(\mathbb{P}^{4}(x) \times \mathbb{P}_{-}^{1}(z)\right)$ is in the base locus of the projection, the images of $W$ and $\tilde{U}$ in $\mathbb{P}^{6}(s)$ coincide. Furthermore $\tilde{U}$ is projected from a point on the plane over the origin, hence its image $U$ has degree one less.

We have seen that the linear syzygies all come from a double point or from a proper trisecant to the scroll $S_{(\lambda: \mu)}$ inside $\mathbb{P}_{(\lambda: \mu)}^{4} \subset \mathbb{P}^{14}(t)$. This $\mathbb{P}_{(\lambda: \mu)}^{4}$ meets the projection center in the origin $o_{(\lambda: \mu)}$ of the elliptic curve $E_{(\lambda: \mu)}$ in $S_{(\lambda: \mu)} . S_{(\lambda: \mu)}$ is 4-tuple in this point so its image $T_{(\lambda: \mu)}$ under $\pi_{T}$ must be a scroll of degree 11 in a $\mathbb{P}_{(\lambda: \mu)}^{3} \subset \mathbb{P}^{6}(s)$. This scroll is 4-tuple along an elliptic quartic curve $F_{(\lambda: \mu)}$, which is the image of $E_{(\lambda: \mu)}$ under the projection from the origin.

The scroll $T_{(\lambda: \mu)}$ sits inside the $\mathbb{P}^{2}$-scroll $U$, which in turn spans $\mathbb{P}^{6}(s)$ since the line $S_{o_{(\lambda: \mu)}}=$ $S_{-}$over the origin and the three planes over the nontrivial 2-torsion points $\operatorname{span} \mathbb{P}^{6}(s)$.

The degree of $\tilde{U}$ is 15 , whence the degree of $U$ is 14 , and any hyperplane which contains $T_{(\lambda: \mu)}$ must intersect $U$ in three more planes. Three planes in $U$ over the points $p_{i}$ do have a double point or a proper trisecant outside the span of $T_{(\lambda: \mu)}$ iff they span at most a hyperplane in $\mathbb{P}^{6}(s)$. This happens exactly when the three planes together with $T_{(\lambda: \mu)}$ span a hyperplane. Now $\tilde{U}$ is the $\mathbb{P}^{2}$-bundle over $E=E_{(\lambda: \mu)}$ of a rank 3 vector bundle with determinant $\mathcal{O}_{E}(15 o), o=o_{E_{(\lambda ; \mu)}}$, and since one gets $U$ by projecting from a point over the origin its rank 3 bundle has determinant $\mathcal{O}_{E}(14 o)$. The rank 2 vector bundle associated to $S_{(\lambda: \mu)}$ has also determinant $\mathcal{O}_{E}(15 o)$, so the bundle associated to $T_{(\lambda: \mu)}$ has determinant $\mathcal{O}_{E}(11 o)$. Therefore three planes in $U$ over the points $p_{i}$ have a double point or a trisecant outside the span of $T_{(\lambda: \mu)}$ precisely when the three points $p_{i}$ add up to the origin in the group law of the elliptic curve.

We can now settle the last two cases.

In case (iv) the three $S_{p_{i}}$ 's are planes which meet $T_{(\lambda: \mu)}$ along lines. The three points do not add up to the origin, thus there are no trisecant lines outside the $\mathbb{P}_{(\lambda ; \mu)}^{3}$ spanned by $T_{(\lambda: \mu)}$. 
On the other hand, the two lines over 2-torsion points do intersect on $T_{(\lambda: \mu)}$, while all three lines have a pencil of trisecant lines on $T_{(\lambda: \mu)}$. This accounts for one quadratic syzygy of type (II) and one of type (III). Of the linear syzygies, the points $\left(p_{i}, q_{i 1}\right)$ arebasepoints of $\rho$, while the points $\left(p_{i}, q_{i 2}\right)$ are mapped to a proper trisecant line. Therefore $\alpha_{1}$ has exactly one genuine quadratic syzygy, which we may choose to be of type (II).

In case (v) the three $S_{p_{i}}$ 's meet in $T_{(\lambda: \mu)}$ as they did in $S_{(\lambda: \mu)}$, and there is one trisecant line to the above planes which lies outside $T_{(\lambda: \mu)}$ (the three points in this case do add up to the origin). So there is only one genuine quadratic syzygy which is is of type (III).

This concludes the analysis of quadratic syzygies corresponding to the trivial character.

For the non-trivial characters the calculations are similar. Again we multiply the matrix $M_{x}(y)$ with a column vector with quadratic entries in the $y$ 's, namely, with the column vector for the trivial character acted on by $\sigma^{j} \tau^{k}$.

We look at the case $(j, k)=(1,2)$, the other cases are similar.

Now the $i^{\text {th }}$ entry of the product matrix is a cubic in the span of

$$
y_{i+1}^{3}, y_{i} y_{i+1} y_{i+2}, y_{i+1} y_{i+3} y_{i+4}, y_{i+2}^{2} y_{i+4}, y_{i+2} y_{i+3}^{2}, y_{i}^{2} y_{i+3}, y_{i} y_{i+4}^{2},
$$

$i=\overline{0,4}$, and its coefficient column vector is

$$
T_{(1,2)}^{\prime}(x, b)=\left(\begin{array}{c}
x_{4} b_{0} \\
x_{4} b_{2}+\left(\xi^{3} x_{0}+\xi^{2} x_{3}\right) b_{1} \\
x_{4} b_{1}+\left(\xi x_{1}+\xi^{4} x_{2}\right) b_{2} \\
\xi x_{1} b_{0}+\xi^{2} x_{3} b_{2} \\
\xi^{4} x_{2} b_{1}+\xi^{2} x_{3} b_{0} \\
\xi^{3} x_{0} b_{2}+\xi^{4} x_{2} b_{0} \\
\xi^{3} x_{0} b_{0}+\xi x_{1} b_{1}
\end{array}\right)
$$

multiplied by $\xi^{i+2} . T_{(1,2)}^{\prime}$ defines a rational map

$$
\Phi_{T_{(1,2)}^{\prime}}: \mathbb{P}^{4}(x) \times \mathbb{P}^{2}(b)--\rightarrow \mathbb{P}^{6}(s),
$$

well-defined outside the codimension 5 set

$$
B_{\Phi_{T_{(1,2)}^{\prime}}}=\left(L_{12}(x) \times \mathbb{P}^{2}(b)\right) \cap\left\{b_{0}^{2}+b_{1} b_{2}=\xi x_{1} b_{0}-\xi^{3} x_{0} b_{2}=\xi x_{1} b_{1}+\xi^{3} x_{0} b_{0}=0\right\},
$$

which is a rational curve projecting bijectively onto the HM-line

$$
L_{12}=\sigma \tau^{2} \mathbb{P}_{-}^{1}=\left\{x_{4}=\xi^{3} x_{0}+\xi^{2} x_{3}=\xi x_{1}+\xi^{4} x_{2}=0\right\} \subset \mathbb{P}^{4}(x)
$$

in the first factor, and onto the conic $\left\{b_{0}^{2}+b_{1} b_{2}=0\right\} \subset \mathbb{P}^{2}(b)$ in the second factor.

The quadratic syzygies which are induced by the linear ones appear with summands of type $M_{p}(y) L_{q}(y)\left(y_{3}, \xi y_{4}, \xi^{2} y_{0}, \xi^{3} y_{1}, \xi^{4} y_{2}\right)^{t}$, with cubic entries as above. This time the coefficient vector of the $i^{\text {th }}$ entry is

$$
T_{(1,2)}^{\prime}(x, z)=\left(\begin{array}{c}
x_{4} z_{4} \\
x_{4}\left(\xi^{4} z_{0}+\xi z_{3}\right)+\left(\xi^{3} x_{0}+\xi^{2} x_{3}\right)\left(\xi^{3} z_{1}+\xi^{2} z_{2}\right) \\
x_{4}\left(\xi^{3} z_{1}+\xi^{2} z_{2}\right)+\left(\xi x_{1}+\xi^{4} x_{2}\right)\left(\xi^{4} z_{0}+\xi z_{3}\right) \\
\xi x_{1} z_{4}+\xi^{2} x_{3}\left(\xi^{4} z_{0}+\xi z_{3}\right) \\
\xi^{4} x_{2}\left(\xi^{3} z_{1}+\xi^{2} z_{2}\right)+\xi^{2} x_{3} z_{4} \\
\xi^{3} x_{0}\left(\xi^{4} z_{0}+\xi z_{3}\right)+\xi^{4} x_{2} z_{4} \\
\xi^{3} x_{0} z_{4}+\xi x_{1}\left(\xi^{3} z_{1}+\xi^{2} z_{2}\right)
\end{array}\right)
$$


multiplied by $\xi^{i+3}$. The linear map

$$
b_{0} \mapsto z_{4}, \quad b_{1} \mapsto\left(\xi^{3} z_{1}+\xi^{2} z_{2}\right), \quad b_{2} \mapsto\left(\xi^{4} z_{0}+\xi z_{3}\right)
$$

gives rise to a commutative diagram similar to the one for the trivial character. Only this time the map from $\mathbb{P}^{14}(t)$ to $\mathbb{P}^{6}(s)$ factors through the projection from the HM-line $L_{12}$, which corresponds to our character.

Since none of the planes spanned by the $p_{i}$ 's of the cases listed in (5.1) meets any HM-line except $\mathbb{P}_{-}^{1}$, the only possible quadratic syzygies of this character come either from double points, or from trisecants of the images of the three planes $\left\{p_{i}\right\} \times \mathbb{P}^{2}(b)$ in $\mathbb{P}^{6}(s)$, which are not inherited from $\mathbb{P}^{14}(t)$. The calculations run as above, except that now we have no new trisecants or double points. For instance, if all the $p_{i}$ 's lie on an elliptic curve, then this is because addition on the elliptic curve should now sum up to a non-trivial 5 -torsion point instead of the origin. In the $\iota$-invariant cases the three planes will span all of $\mathbb{P}^{6}(s)$, without intersecting each other, and therefore they will have a pencil of trisecant lines, all of which are inherited from $\mathbb{P}^{14}(t)$.

Proposition 5.12. In the above cases of (5.1) with 10 first order linear syzygies $\left(L_{q_{i j}}\right)$ there are no second order quadratic syzygies of $\alpha_{1}$.

Proof. The number of such syzygies, if they exist at all, is a multiple of 5. More precisely, from Proposition 5.10, Proposition 5.11 and the double complex of the Koszul cohomology we may deduce that the linear and quadratic part of the minimal free resolution of $M$ contains a subcomplex

$$
\begin{aligned}
& V_{3} \otimes R \stackrel{\left(M_{p_{i}}\right)}{\leftarrow} 3 V_{1} \otimes R(-1) \quad \stackrel{\left(L_{q_{i j}}, L_{i}^{\prime}\right)}{\nwarrow} \quad 2 V_{0} \otimes R(-2) \quad a I \otimes R(-3) \\
& (a+b) I \otimes R(-3) \stackrel{\oplus}{\oplus} \stackrel{\oplus}{\oplus} \otimes R(-4),
\end{aligned}
$$

where $a, b \leq 2$ and $L_{i}^{\prime}=\left(L_{r_{i k}}^{\prime}\right), i=\overline{1,3}$, is as in the proof of Proposition 5.11. Hence 5 second order quadratic syzygies are given by a relation

$$
L_{q_{i 1}}(y) P_{1}(y)+L_{q_{i 2}}(y) P_{2}(y)+L_{i}^{\prime}(y) P_{3}(y)=0, \quad i=\overline{1,3},
$$

where $P_{1}$ and $P_{2}$ are $5 \times 5$-matrices with quadratic entries and $P_{3}$ is an $(a+b) \times 5$-matrix with linear entries. We want to show that $\mathrm{c}=0$ or equivalently that such a relation is trivial or depends on the second order linear syzygies.

We consider first the cases (ii)', (iv) and (v). In these cases $L_{q_{31}}=0$ by Proposition 5.8 and $(a+b)=1$ by Proposition 5.10. Thus the third of the above matrix equations is just

$$
L_{q_{32}}(y) P_{2}(y)+L_{3}^{\prime}(y) P_{3}(y)=0,
$$

and $L_{3}^{\prime}(y) P_{3}(y)$ has generic rank $\leq 1$. Hence $L_{q_{32}}(y) P_{2}(y)$ must also have generic rank $\leq 1$. But in all the above cases $\operatorname{det} L_{q_{32}}$ is a genuine quintic polynomial, thus $L_{q_{32}}$ has generic rank 5 which means that $P_{2}(y)$ must have rank $\leq 1$. If $P_{2}(y)$ is non-zero it represents an element in

$$
\mathbb{P}\left(\operatorname{Hom}_{H_{5}}\left(V_{2} \otimes R(-4), V_{0} \otimes R(-2)\right)\right) \cong \mathbb{P}\left(\operatorname{Hom}_{H_{5}}\left(V_{2}, V_{0} \otimes S^{2} V_{0}^{*}\right)\right),
$$


and is thus of type

$$
P_{2}(y)=b_{i-j} y_{3 i+3 j}^{2}+c_{i-j} y_{3 i+3 j+2} y_{3 i+3 j+3}+d_{i-j} y_{3 i+3 j+1} y_{3 i+3 j+4}, \quad i, j \in \mathbb{Z}_{5}
$$

where $\left(b_{0}: \cdots: d_{4}\right)$ is a parameter point in $\mathbb{P}^{14}$. In this case a check on the $2 \times 2$-minors of $P_{2}(y)$ shows that $P_{2}(y)$ factors through $\chi \otimes R(-3)$ for some character $\chi$ of $H_{5}$, i.e., $P_{2}(y)$ is a product $P_{2}(y)=P_{2}^{\prime}(y) P_{2}^{\prime \prime}(y)$, where $P_{2}^{\prime}(y)=\left(\xi^{j i} y_{4 k+i}\right)_{i \in \mathbb{Z}_{5}}^{t}$ and $P_{2}^{\prime \prime}(y)=\left(\xi^{4 j i} y_{k+i}\right)_{i \in \mathbb{Z}_{5}}$ for some $(j, k) \in \mathbb{Z}_{5} \times \mathbb{Z}_{5}$ (of course this follows also from the factoriality of the polynomial ring). By comparing coefficients in (5.14) we see that in fact $\chi$ must be the trivial character, i.e., $P_{2}^{\prime \prime}(y)=P_{3}(y)=[y]=\left(y_{0}, \ldots, y_{4}\right)$ and $P_{2}^{\prime}(y)=[y]^{t}$.

In case $(\mathrm{v}) L_{3}^{\prime}$ is non-zero by the proof of Proposition 5.11. It follows that $P_{2}(y)$ and $P_{3}(y)$ are zero, since otherwise the first order quadratic syzygies would depend on the linear ones. Indeed, this can be seen by rewriting (5.14) as

$$
L_{q_{32}}^{\prime}(y) P_{2}^{\prime \prime}(y)+L_{3}^{\prime}(y) P_{3}(y)=0
$$

with $L_{q_{32}}^{\prime}=L_{q_{32}} P_{2}^{\prime}(y)$. Then also $P_{1}(y)=0$, since $\operatorname{det} L_{q_{11}}$ (and also $\operatorname{det} L_{q_{21}}$ ) are not vanishing identically. Notice that in this case there are no second order linear syzygies.

In the cases (ii)' and (iv) there is precisely one second order linear syzygy. We may suppose by the proof of Proposition 5.11 that $L_{3}^{\prime}(y)$ and thus also $P_{2}(y)$ are zero. Hence (5.13) yields

$$
\left(\lambda L_{q_{11}}(y)+\mu L_{q_{21}}(y)\right) P_{1}(y)+\left(\lambda L_{1}^{\prime}(y)+\mu L_{2}^{\prime}(y)\right) P_{3}(y)=0
$$

for all $(\lambda: \mu) \in \mathbb{P}^{1} . \lambda L_{q_{11}}(y)+\mu L_{q_{21}}(y)$ has generic rank 4 (being skew-symmetric) but nevertheless moving kernel with respect to $(\lambda: \mu) \in \mathbb{P}^{1}$. Therefore a similar argument as above allows us to conclude that $P_{1}(y)=0$ or $P_{1}(y)=[y]^{t}[y]$. In the latter case $\left(\lambda L_{q_{11}}(y)+\right.$ $\left.\mu L_{q_{21}}(y)\right)[y]^{t}=0$ implies $P_{3}(y)=0$, so $\left(P_{1}, P_{2}, P_{3}\right)^{t}=\left([y]^{t}[y], 0,0\right)$ depends on the second order linear syzygies. In the first case $P_{1}=P_{2}=P_{3}=0$.

Case (i) is well known (see [De2], [Ma3] and [Ma4]) and can be worked out directly. In case (iii) we may suppose that $L_{1}^{\prime}=0$ by the proof of Proposition 5.11 (compare the proof of Proposition 8.2 for $L_{2}^{\prime}$ and $L_{3}^{\prime}$ ). Since everything is very explicit, this case can also be worked out directly.

\section{SYZYGY CONSTRUCTION OF BIELLIPTIC SURFACES}

In this section we prove the main results of this paper. Namely, we describe the structure of the Hartshorne-Rao modules and the syzygies of the minimal bielliptic surfaces in $\mathbb{P}^{4}$ and of the bielliptic surfaces of degree 15 studied in [ADHPR]. At the same time we explain how to construct these surfaces via syzygies. We are not able to show directly that our constructions do lead to smooth surfaces. To overcome this difficulty we make use of a detailed classification of the $\mathrm{H}_{5}$-invariant surfaces of small degree lying on either the trisecant variety of an elliptic quintic scroll in $\mathbb{P}^{4}$, or on the secant variety of an elliptic normal curve in $\mathbb{P}^{4}$.

Proposition 6.1. Let $V_{(\lambda: \mu)}=\operatorname{Trisec} Q_{(\lambda: \mu)} \subset \mathbb{P}^{4},(\lambda: \mu) \in \mathbb{P}_{-}^{1} \backslash \Lambda$, be the trisecant variety of the elliptic scroll $Q_{(\lambda: \mu)}$ and let $X \subset V_{(\lambda ; \mu)}$ be a reduced $H_{5}$-invariant surface of degree $d \leq 10$ lying on this quintic hypersurface. Then either

(i) $d=5$ and $X$ is the elliptic scroll $Q_{(\lambda: \mu)}$, or 
(ii) $d=10$ and $X$ is either a minimal abelian surface in the pencil $\left|A_{K}\right|$, or one of the four singular translation scrolls in this pencil (see [ADHPR] and Remark 4.11), or a minimal bielliptic surface $B_{(i, j)}$.

Proof. Let $\tilde{V}$ denote the normalization of $V_{(\lambda: \mu)}$. Recall from [ADHPR, Proposition 24 and Proposition 26] that $\tilde{V}$ can be identified with a $\mathbb{P}^{2}$-bundle $\mathbb{P}_{E}^{2}$ over $E$, the elliptic curve which is the base of the scroll $Q_{(\lambda: \mu)}$. The group $G_{5}$ acts on $V_{(\lambda: \mu)}$ and thus also on $\tilde{V}$, and furthermore the projection $p: \tilde{V} \rightarrow E$ is equivariant under this action. The generators of Num $\tilde{V}$ are $F$, the class of a fiber of $p$, and $H$, the class of $\mathcal{O}_{\mathbb{P}_{E}^{2}}(1)$. The normalization map onto $V_{(\lambda: \mu)} \subset \mathbb{P}^{4}$ is induced by $\mathcal{O}_{\mathbb{P}_{E}^{2}}(1)$, so we compute that $H^{3}=5, H^{2} \cdot F=1$ and $H \cdot F^{2}=F^{3}=0$. On the other side, the canonical class $K$ on $\tilde{V}$ is numerically equivalent to $-3 H+5 F$, and in fact $|-K|$ corresponds to the pencil $\left|A_{K}\right|$ on $V_{(\lambda: \mu)}$ (see [ADHPR, Theorem 30]).

Assume first that $X$ is not contained inside the non-normal locus of $V_{(\lambda: \mu)}$, and let $X_{0}$ denote the strict transform of $X$ on $\tilde{V}$. By Heisenberg invariance the degree of $X$ is divisible by 5 , thus we need to find the integral solutions of $X_{0} \equiv a H+b F$, with $H^{2} \cdot X_{0}=5 m, m=1,2$. The coefficients $a$ and $b$ satisfy the inequalities

$$
a=H \cdot X_{0} \cdot F \geq 0, \quad \text { and } \quad 10 a+3 b=H \cdot X_{0} \cdot(3 H-5 F) \geq 0
$$

Moreover, $X_{0}$ must intersect the pullback on $\tilde{V}$ of any curve in the antibicanonical pencil on $Q_{(\lambda: \mu)}$ in a scheme whose length is a multiple of 25 , since these are all $H_{5}$-invariant. If $C_{K}$ is the base curve $K^{2}$ of the pencil of anticanonical surfaces on $\tilde{V}$, then the numerical class of the curves in the antibicanonical pencil on $Q_{(\lambda: \mu)}$ is $5 H-C_{K}$. So their pullback to $\tilde{V}$ is equivalent to $(3 H-5 F) \cdot(5 H-(3 H-5 F))=(3 H-5 F) \cdot(2 H+5 F)$. Thus we get that $35 a+6 b$ is a multiple of 25 . Combining this with the above $H^{2} \cdot X_{0}=5 a+b=5 m$, for $m=1,2$, we get that

$$
10 a+b \text { is a multiple of } 25 \text {. }
$$

For $m=1$ there are no numerical solutions. In case $m=2$ we are left with $(a, b)=(3,-5)$. This is the numerical class of the anticanonical bundle on $\tilde{V}$ and from [ADHPR, Lemma 3, Lemma 4 and Theorem 30] it follows that the only reduced surfaces of degree 10 in this class are those listed at (ii) in the statement of the lemma.

At last (i) follows from the fact that the scroll $Q_{(\lambda: \mu)}$ coincides with the non-normal locus of $V_{(\lambda: \mu)}$.

Proposition 6.2. Let $W_{(\lambda: \mu)}=\operatorname{Sec} E_{(\lambda: \mu)} \subset \mathbb{P}^{4},(\lambda: \mu) \in \mathbb{P}_{-}^{1} \backslash \Lambda$, be the secant variety of the elliptic curve $E_{(\lambda: \mu)} \subset \mathbb{P}^{4}$, and let $X \subset W_{(\lambda: \mu)}$ be a reduced $H_{5}$-invariant surface of degree $d \leq 15$ lying on this quintic hypersurface. Then either

(i) $d=5$ and $X$ is one of the 3 translation scrolls $\Sigma_{(\lambda: \mu)}\left(\tau_{i}\right)$, where $\tau_{i} \in E_{(\lambda: \mu)}$ is a nontrivial 2-torsion point, or

(ii) $d=10$ and $X$ is either a singular translation scroll (see [BHM2] and [ADHPR, proof of Proposition 36]), or the union $\Sigma_{(\lambda: \mu)}\left(\tau_{1}\right) \cup \Sigma_{(\lambda: \mu)}\left(\tau_{2}\right)$, where $\tau_{i} \in E_{(\lambda: \mu)}, i=1$, are distinct non-trivial 2-torsion points, or

(iii) $d=15$ and $X$ is either a non-minimal abelian surface $\tilde{A}_{K}$ (see [ADHPR, Theorem $32]$ ), or a non-minimal bielliptic surface $\tilde{B}_{(i, j)}$ (see again [ADHPR]), or the union of a 
degenerate abelian surface of degree 10 and an elliptic quintic scroll $\Sigma_{(\lambda: \mu)}\left(\tau_{i}\right)$, or the singular scroll $S_{(\lambda: \mu)}$ (see Proposition 4.16), or the union $\Sigma_{(\lambda ; \mu)}\left(\tau_{1}\right) \cup \Sigma_{(\lambda ; \mu)}\left(\tau_{2}\right) \cup \Sigma_{(\lambda: \mu)}\left(\tau_{3}\right)$, where $\tau_{i} \in E_{(\lambda: \mu)}, i=\overline{1,3}$, are the non-trivial 2-torsion points. Among these surfaces the bielliptic are the only ones that are not also $\iota$-invariant.

Proof. Let $\tilde{W}$ be the blow-up of $W_{(\lambda: \mu)}$ along the curve $E_{(\lambda: \mu)}$. Then $\tilde{W}$ has the structure of a $\mathbb{P}^{1}$-bundle over the symmetric product $\Sigma$ of $E_{(\lambda: \mu)}$ (see [ADHPR, proof of Proposition 36] for more details). Let $X_{0}$ be the strict transform of $X$ on $\tilde{W}$. Now $G_{5}$ acts on $\tilde{W}$ and both projections $p: \tilde{W} \rightarrow \Sigma$ and $q: \tilde{W} \rightarrow E_{(\lambda ; \mu)}$ are equivariant under this action. Let $F$ denote the class of a fiber of $q$. Such a fiber is mapped to a rational cubic scroll in $\mathbb{P}^{4}$. Let $B$ denote the scroll in $\tilde{W}$ over a section of $\Sigma$ with self-intersection 1 . The image of $B$ on $W_{(\lambda: \mu)}$ is the cone of secants to $E_{(\lambda: \mu)}$ through a given point on it. In particular, it is a cone over an elliptic curve of degree 4 . Therefore $B$ has a unique section with self-intersection -4 which is contracted to a point on $E_{(\lambda: \mu)}$. Let $S$ be the section in $\tilde{W}$ over $\Sigma$ which consists of the directrices in each of the F's. The image of $S$ in $\operatorname{Sec} E_{(\lambda ; \mu)}$ is the scroll $S_{(\lambda ; \mu)}$.

If $H$ on $\tilde{W}$ is the pullback of the class of a hyperplane section of $W_{(\lambda ; \mu)}$, then $H \equiv S+2 B-6 F$ in Num $\tilde{W}$. Furthermore, if $K$ is the class of the dualizing sheaf of $\tilde{W}$ and $K_{\Sigma}$ the pullback of the canonical class on $\Sigma$, then $K \equiv-2 S-3 B+14 F$ and $K_{\Sigma} \equiv-2 B+F$. The exceptional surface $Y$ on $\tilde{W}$ is anticanonical and has the structure of a product $E_{(\lambda: \mu)} \times E_{(\lambda ; \mu)}$ (see [ADHPR, p. 886]). In particular, every curve on it has non-negative selfintersection.

The intersection numbers on $\tilde{W}$ are: $H^{2} \cdot F=3, \quad H^{2} \cdot S=15, \quad H^{2} \cdot B=4$. Moreover, $B^{2} \cdot F=B \cdot F^{2}=H \cdot F^{2}=F^{3}=B^{3}=0$, while $H \cdot B \cdot F=H \cdot B^{2}=1$, and $B \cdot S^{2}=$ $12, \quad B \cdot F \cdot S=-F \cdot S^{2}=B^{2} \cdot S=1, \quad S^{3}=-25$. We are looking for integral solutions of $X_{0} \equiv a F+b B+c S, H^{2} \cdot X_{0}=5 m, m=\overline{1,3}$, with coefficients satisfying the inequalities

$$
c=X_{0} \cdot B^{2} \geq 0, \quad b-c=X_{0} \cdot S \cdot F \geq 0 \quad \text { or } \quad a=b=0, c=1,
$$

and

$$
a+b+4 c=X_{0} \cdot B \cdot(S+2 B-10 F) \geq 0,
$$

where the last inequality comes from the fact that $B \cdot(H-4 F)=B \cdot(S+2 B-10 F)$ is the section with selfintersection -4 on any surface in the class $B$. Furthermore,

$$
2 a b+a c+2 b+13 b c=X_{0}^{2} \cdot(2 S+3 B-14 F) \geq 0
$$

since every curve on the exceptional surface has non-negative selfintersection. Finally,

$$
2 a+b \text { is a multiple of } 25 \text {. }
$$

Indeed, consider the 3 quintic elliptic scrolls which are the 2-torsion translation scrolls of $E_{(\lambda: \mu)}$. The pullback of any antibicanonical curve on one of these scrolls belong to the class $(2 B-F) \cdot(4 S+2 B-26 F)$, and it intersects $X_{0}$ in a multiple of 25 points, unless $X_{0}$ is the union of the three scrolls.

For $m=1$ only the triple $(a, b, c)=(-1,2,0)$ satisfies our conditions. The surfaces $X_{0} \equiv$ $-F+2 B$ are the pullbacks of numerical anticanonical curves on $\Sigma$. It follows from [CC] that $X$ is necessarily one of the three elliptic quintic scrolls $\Sigma_{(\lambda: \mu)}\left(\tau_{i}\right) \subset W_{(\lambda: \mu)}$, where $\tau_{i} \in E_{(\lambda: \mu)}$ is a non-trivial 2-torsion point. 
For $m=2$ we are left with $(a, b, c)=(-2,4,0)$. Invariant divisors in the corresponding numerical class are either singular translation scrolls of degree 10 (see [HM], or [Hu1, Theorem VII.3.1]), or the union of two elliptic quintic scrolls $\Sigma_{(\lambda: \mu)}\left(\tau_{1}\right) \cup \Sigma_{(\lambda: \mu)}\left(\tau_{2}\right)$.

Finally, for $m=3$ the numerical solutions are

$$
(a, b, c) \in\{(-3,6,0),(0,0,1),(-14,3,3)\} .
$$

A surface in the first numerical class is a pullback of an antitricanonical curve on $\Sigma$, and so it is an $\iota$-invariant scheme ("translation scroll") of degree 15. Such a surface is either the union of a degenerate abelian surface of degree 10 (see [BHM2]) and a quintic elliptic scroll $\Sigma_{(\lambda: \mu)}\left(\tau_{i}\right)$, or the union of all the three quintic elliptic scrolls $\Sigma_{(\lambda: \mu)}\left(\tau_{i}\right)$. The second class corresponds to the surface $S_{(\lambda: \mu)}$ of degree 15, while the last solution corresponds to the pullback of numerically anticanonical surfaces on the variety $\tilde{V}$ of the proof of Proposition 6.1 via the blow-up map $\tilde{W} \rightarrow \tilde{V}$ with exceptional divisor $S$ (see also [ADHPR, Proposition 28 and 36]. Here the bielliptic surfaces are the only ones that are not also $\iota$-invariant.

Theorem 6.3. Let $M=\bigoplus_{k \geq 0} M_{k}$ be the $H_{5}$-invariant graded $R$-module presented by

$$
\alpha_{1}=\left(M_{\tau_{1}}(y), M_{\tau_{2}}(y), M_{\tau_{3}+\rho}(y)\right),
$$

where $\tau_{i}, i=\overline{1,3}$, are the non-trivial 2-torsion points and $\rho$ is a non-trivial 3-torsion point on the elliptic normal curve $E_{(\lambda: \mu)} \subset \mathbb{P}^{4},(\lambda: \mu) \in \mathbb{P}_{-}^{1} \backslash \Lambda$. Then $S y z_{1}(M(1))$ is a vector bundle of rank 10 and there exists a sheaf monomorphism

$$
\varphi: 5 \mathcal{O}(-1) \oplus \Omega^{3}(3) \rightarrow \mathcal{S} y z_{1}(M(1))
$$

Such a morphism drops rank along a smooth minimal bielliptic surface $B$ of degree 10, and it is uniquely determined up to isomorphisms, hence $B$ is uniquely determined by $M$. Moreover, $B$ is contained in precisely one quintic hypersurface. This is the trisecant variety $\operatorname{Trisec} Q=\left\{\operatorname{det} L_{q}(y)=0\right\}$ defined by a point $q$ on the numerically anticanonical curve $E_{(\lambda: \mu)}\left(\tau_{3}\right) \subset Q_{(\lambda: \mu)}$ not on a HM-line (compare 4.3, 4.12, 4.20 and 4.21). Conversely, any smooth minimal bielliptic surface $B \subset \mathbb{P}^{4}$ can be obtained in this way (up to coordinate transformations). In particular, there are 8 different bielliptic surfaces on Trisec $Q$, corresponding to the eight different choices of a non-trivial 3-torsion point on $E_{(\lambda: \mu)}$.

Proof. First of all $M$ is artinian by Proposition 5.5, whence $\delta y z_{1}(M(1))$ is a vector bundle. Now $\alpha_{1}$ has exactly ten first order linear syzygies by Proposition 5.8, one first order quadratic syzygy by Proposition 5.11, one second order linear syzygy by Proposition 5.10 and no second order quadratic syzygies by Proposition 5.12. In particular, $M$ has Hilbert function $(5,10,10)$ and a minimal free resolution of type

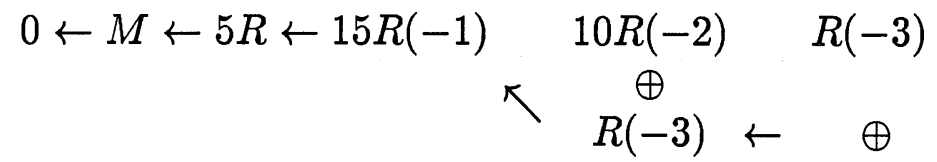

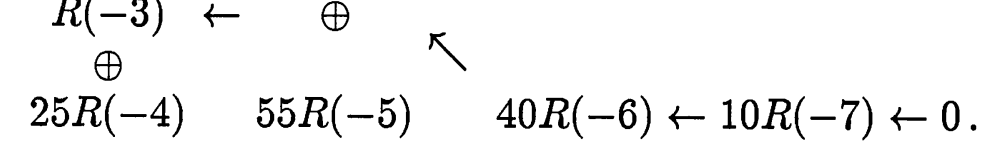

It follows readily that there exists, up to isomorphisms, a uniquely determined sheaf mono- 
morphism $\varphi \in \operatorname{Hom}\left(5 \mathcal{O}(-1) \oplus \Omega^{3}(3), \delta y z_{1}(M(1))\right)$, and that this morphism drops rank in the expected codimension 2. Thus the maximal minors of $\varphi$ cut out a locally Cohen-Macaulay surface $B$ whose ideal sheaf $\partial_{B}$ has a resolution

$$
0 \rightarrow 5 \mathcal{O}(-1) \oplus \Omega^{3}(3) \stackrel{\varphi}{\rightarrow} \delta y z_{1}(M(1)) \rightarrow \partial_{B}(3) \rightarrow 0 .
$$

By Porteus' formula $\operatorname{deg} B=c_{2}\left(S y z_{1}(M(1))\right)-c_{2}\left(5 \mathcal{O}(-1) \oplus \Omega^{3}(3)\right)=10$, while, in case $B$ is reduced and irreducible, Riemann-Roch on $\mathbb{P}^{3}$ yields $\pi(B)=6$ for the arithmetic sectional genus. On the other side, $\chi\left(\mathcal{O}_{B}\right)=0$ and $h^{1}\left(\mathcal{O}_{B}\right)=h^{3}\left(\Omega^{3}\right)=1$, thus $B$ has the same numerical invariants as a minimal bielliptic surface in $\mathbb{P}^{4}$. In order to see that $B$ is indeed a smooth bielliptic surface we will use Proposition 6.1.

We will first show that $B$ is contained in $\operatorname{Trisec} Q$. From the mapping cone

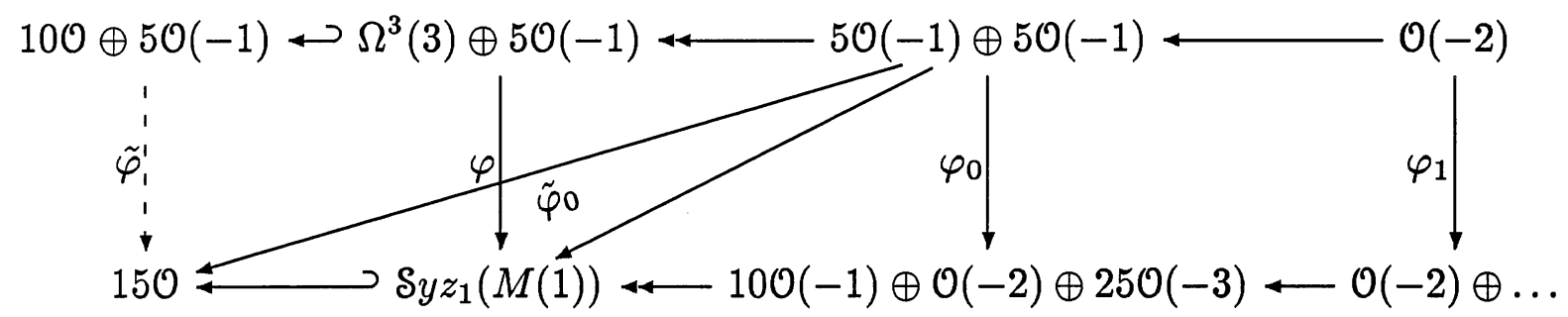

between the minimal free resolutions of $5 \mathcal{O}(-1) \oplus \Omega^{3}(3)$ and $\delta y z_{1}(M(1))$ we see that $B$ is contained in precisely one quintic hypersurface in $\mathbb{P}^{4}$. In fact we can say more. The composition $\tilde{\varphi}_{0}: 5 \mathcal{O}(-1) \oplus 5 \mathcal{O}(-1) \rightarrow 15 \mathcal{O}$ in the above diagram is given by the first order linear syzygies of $\alpha_{1}$. Since $h^{1}\left(\Omega^{2}(3)\right)=0$ we may lift $\tilde{\varphi}_{0}$ to a morphism $\tilde{\varphi} \in \operatorname{Hom}(10 \mathcal{\oplus} \oplus$ $5 \mathcal{O}(-1), 15 \mathcal{O})$ whose constant part is of maximal rank. After moding out constants in both sides we are left with a $5 \times 5$-matrix $\tilde{L}$ with linear entries whose determinant is exactly the equation of the unique quintic containing $B$. We may suppose by Proposition 5.8, (iv) that the first order linear syzygies of $\alpha_{1}$ are given by a block of syzygy matrices

$$
\left(\begin{array}{cc}
L_{q_{11}}(y) & L_{q_{12}}(y) \\
L_{q_{21}}(y) & L_{q_{22}}(y) \\
0 & L_{q_{32}}(y)
\end{array}\right)
$$

with $q_{i 1} \in \mathbb{P}_{-}^{1}, i=1,2$, and $q_{32} \in E_{(\lambda: \mu)}\left(\tau_{3}\right)$ not on a HM-line. Moreover, by Proposition $5.10, \alpha_{1}$ has only one second order linear syzygy and this is of type $\left([y]^{t}, 0\right)$. We therefore may suppose that our matrix $\tilde{L}$ coincides with $L_{q_{32}}(y)$, whence $B$ lies on the quintic hypersurface $\operatorname{Trisec} Q=\left\{\operatorname{det} L_{q_{32}}(y)=0\right\}$ (compare Proposition 4.12, (i)).

We will show next that $B$ is reduced. Assume the contrary and let $B_{\text {red }}$ denote the reduced support of $B$. Then $B_{\text {red }} \subset \operatorname{Trisec} Q$ is a $H_{5}$-invariant surface of degree 5 , hence $B_{\text {red }}$ coincides with $Q$ by Proposition 6.1, and $B$ is a locally Cohen-Macaulay double structure on $Q$. It follows that $B$ is necessarily a local complete intersection (see e.g. [Ma2, proof of Theorem 1]), and that it comes through a Ferrand doubling [Fe]. In other words, there is an exact sequence

$$
0 \rightarrow \partial_{B} / \partial_{Q}^{2} \rightarrow \partial_{Q} / \partial_{Q}^{2} \rightarrow \mathcal{L} \rightarrow 0,
$$

where $\mathcal{L}$ is a line bundle on $Q$. Since $\mathcal{L}=\partial_{Q} / \partial_{B}$ we also have an exact sequence

$$
0 \rightarrow \mathcal{L} \rightarrow \mathcal{O}_{B} \rightarrow \mathcal{O}_{Q} \rightarrow 0
$$


Let $C_{0}$ and $f$ be the generators of $\operatorname{Num} Q$, and write $\mathcal{L} \equiv a C_{0}+b f$. If follows from (6.4) that the twisted normal bundle $\mathcal{N}_{Q} \otimes \mathcal{L}$ of $Q$ in $\mathbb{P}^{4}$ has a nowhere vanishing section. In particular,

$$
0=c_{2}\left(\mathcal{N}_{Q} \otimes \mathcal{L}\right)=25+14 a+3 b+a^{2}+2 a b .
$$

On the other hand, $\chi(\mathcal{L})=\chi\left(\mathcal{O}_{B}\right)-\chi\left(\mathcal{O}_{Q}\right)=0$ by $(6.5)$ since $B$ has the numerical type of a bielliptic surface. Thus Riemann-Roch implies that

$$
\mathcal{L}^{2}=K_{Q} \cdot \mathcal{L}, \quad \text { i.e., } \quad a^{2}+2 a b=-a-2 b,
$$

where $K_{Q}$ denotes the canonical class on $Q$. The last equation gives that either $a=-2 b$, or $a=-1$. In conclusion, there are exactly 2 solutions for these equations, namely

$$
\mathcal{L} \equiv-2 C_{0}+f \quad \text { and } \quad \mathcal{L} \equiv-C_{0}-12 f .
$$

Since we already know all the line bundles of numerical type $\mathcal{L} \equiv-2 C_{0}+f$ it is easy to deduce (cf. the proof of [HV, Proposition 4]) that in the first case the only such doublings are the HM-surfaces in [HV]. This is a contradiction, since the HM-surfaces are of the numerical type of an abelian surface and thus in particular have different cohomology. The second case can be ruled out by taking global sections in the exact sequence

$$
0 \rightarrow \partial_{B} / \mathcal{J}_{Q}^{2}(5) \rightarrow \partial_{Q} / \partial_{Q}^{2}(5) \rightarrow \mathcal{L}(5) \rightarrow 0
$$

$\partial_{B} / \partial_{Q}^{2}(5)$ has no sections since both $B$ and the first infinitesimal neighborhood of the scroll $Q$ are contained in the same unique quintic, while $\mathcal{L}(5)$ has at most 2 sections since $\mathcal{L}(5) \equiv$ $-2 K$. But this is a contradiction since $H^{0} \partial_{Q} / \partial_{Q}^{2}(5)=50$.

In conclusion, $B$ is a reduced $H_{5}$-invariant surface of degree 10 on $\operatorname{Trisec} Q$ which is not $\iota$-invariant. Thus it must be a smooth minimal bielliptic surface by Proposition 6.1.

The converse statement is clear from [ADHPR] and Proposition 3.7.

Theorem 6.6. Let $M^{\prime}=\bigoplus_{k \geq 0} M_{k}^{\prime}$ be the $H_{5}$-invariant graded $R$-module presented by

$$
\alpha_{1}=\left(M_{\tau_{1}+\rho}(y), M_{\tau_{2}+\rho}(y), M_{\tau_{3}+\rho}(y)\right)
$$

where $\tau_{i}, i=\overline{1,3}$, are the non-trivial 2-torsion points and $\rho$ is a non-trivial 3-torsion point on the elliptic normal curve $E_{(\lambda: \mu)} \subset \mathbb{P}^{4},(\lambda: \mu) \in \mathbb{P}_{-}^{1} \backslash \Lambda$. Then:

(i) $M^{\prime}$ has Hilbert function $(5,10,10,1)$ and a minimal free resolution of type

$$
\begin{aligned}
& 0 \leftarrow M^{\prime} \leftarrow 5 R \leftarrow 15 R(-1) \quad 10 R(-2) \\
& \nwarrow \quad \stackrel{\oplus}{R(-3)}
\end{aligned}
$$

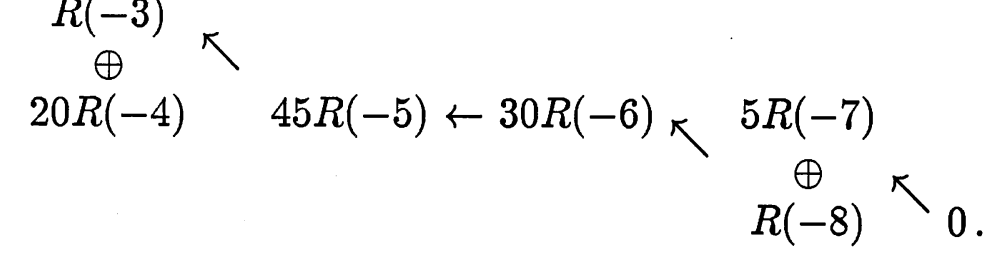

(ii) $S y z_{2}\left(\left(M^{\prime}\right)^{\vee}(3)\right)$ is a vector bundle of rank 21 and there exists a sheaf monomorphism

$$
\varphi: 20 \mathcal{O}(-1) \rightarrow \mathcal{S} y z_{2}\left(\left(M^{\prime}\right)^{\vee}(3)\right)
$$


Such a morphism drops rank along a smooth non-minimal bielliptic surface $B^{\prime}$ of degree 15 , and it is uniquely determined up to isomorphisms, hence $B^{\prime}$ is uniquely determined by $M^{\prime}$. Moreover, $B^{\prime}$ is contained in precisely one quintic hypersurface, namely $\operatorname{Sec} E_{(\lambda: \mu)}$, and the ideal sheaf $\mathcal{J}_{B^{\prime}}$ has a minimal free resolution of type

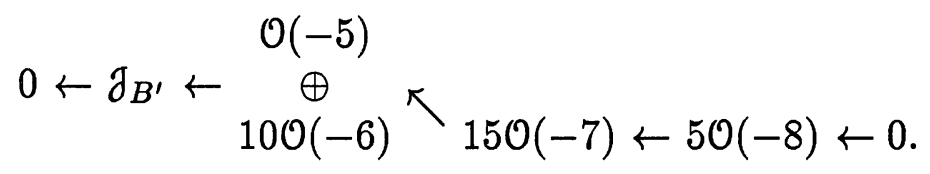

Proof. As in the proof of Theorem 6.3 we deduce that $M^{\prime}$ is artinian, that it has Hilbert function $(5,10,10,1)$ (use Gotzmann's persistence theorem [Go]), and a minimal free resolution as stated above. In particular, $\delta y z_{2}\left(\left(M^{\prime}\right)^{\vee}(3)\right)$ is a rank 21 vector bundle, and it follows that there exists, up to isomorphisms, a uniquely determined sheaf monomorphism

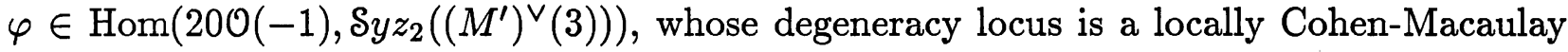
scheme $B^{\prime}$ of codimension 2 . From the exact sequence

$$
0 \rightarrow 20 \mathcal{O}(-1) \stackrel{\varphi}{\rightarrow} \delta y z_{2}\left(\left(M^{\prime}\right)^{\vee}(3)\right) \rightarrow \mathcal{J}_{B^{\prime}}(3) \rightarrow 0
$$

we deduce as in the proof of Theorem 6.3 that $\mathcal{J}_{B^{\prime}}$ has the syzygies as claimed, that $\operatorname{deg} B^{\prime}=$ 15 , that, in case $B^{\prime}$ is reduced and irreducible, $\pi\left(B^{\prime}\right)=21$, and moreover that $\chi\left(\mathcal{O}_{B^{\prime}}\right)=0$ and $h^{1}\left(\mathcal{O}_{B^{\prime}}\right)=1$. In order to see that $B^{\prime}$ is indeed a smooth non-minimal bielliptic surface we will use Proposition 6.2.

We will first show that $B^{\prime}$ is contained in $\operatorname{Sec} E_{(\lambda: \mu)}$. We may suppose by Proposition 5.8, (v) that the first order linear syzygies of $\alpha_{1}$ are given by a block of syzygy matrices

$$
\left(\begin{array}{cc}
L_{q_{1}}(y) & L_{q_{1}}(y) \\
L_{q_{2}}(y) & 0 \\
0 & L_{q_{3}}(y)
\end{array}\right)
$$

where the common determinant $\gamma$ of all 4 non-zero matrices $L_{q_{i}}(y)$ defines $\operatorname{Sec} E_{(\lambda: \mu)}$. The first order linear syzygies together with the first order quadratic syzygy of $\alpha_{1}$ form a $15 \times 11$ matrix whose transpose is the presentation matrix of the ideal $I_{B^{\prime}}$ of $B^{\prime}$. It follows that $I_{B^{\prime}}$ contains all the $10 \times 10$-minors of this $15 \times 11$-matrix. In particular, $I_{B^{\prime}}$ contains $\operatorname{det} L_{q_{2}} \cdot \operatorname{det} L_{q_{3}}$, which is the square of $\gamma$. But we have more: $I_{B^{\prime}}$ contains the product of $\gamma$ by any $5 \times 5$-minor of any $10 \times 5$-matrix formed by two of the three blocks $L_{q_{i}}$. That the unique quintic containing $B^{\prime}$ is $\operatorname{Sec} E_{(\lambda: \mu)}=\{\operatorname{det} \gamma=0\}$ follows therefore immediately from the

Claim. The $5 \times 5$-minors of all three $10 \times 5$-matrices

$$
\left(\begin{array}{l}
L_{q_{i}}(y) \\
L_{q_{j}}(y)
\end{array}\right)
$$

vanish at most on a curve.

Proof of Claim. Let $y$ be a point where all these minors vanish. We may assume that $y$ lies on Sec $E_{(\lambda: \mu)}=\{\gamma=0\}$ outside the curve $E_{(\lambda: \mu)}$, thus we may assume that $L_{q_{1}}(y)$ has rank 4. We argue as in the proofs of Lemmas 5.3, 5.4 and Proposition 5.5. In this case let

$$
L(z, y)\left(\lambda_{0}, \ldots, \lambda_{4}\right)^{t}=0
$$


be the unique relation between the columns of $L(z, y)$ for $z=q_{1}$. The rank condition on the $10 \times 5$-matrices now says that

$$
L(z, y)\left(\lambda_{0}, \ldots, \lambda_{4}\right)^{t}=0
$$

for $z=q_{i}, i=\overline{1,3}$. Thus the linear span of the three points $q_{i}$ is contained in $\operatorname{det} L(z, y)=0$ for the given point $y$. Now this linear span is a plane: The points $q_{i}=\rho+\tau_{i}$ on $E_{(\lambda: \mu)}^{\prime}$ may as points on $Q_{(\lambda: \mu)}$ be identified with the pair of points $\left(\rho, \tau_{i}\right)$ when we identify $Q_{(\lambda: \mu)}$ with the symmetric product of $E_{(\lambda: \mu)}$. Therefore they lie on the same plane cubic curve on the quintic scroll, namely the curve of points with one coordinate equal to $\rho$. It follows from the argument at the end of Section 2 in [ADHPR] that the three points on this curve are collinear iff the sum $\tau_{1}+\tau_{2}+\tau_{3}=-\rho$. Therefore the $q_{i}$ span the plane of the cubic curve. This is a plane in the trisecant scroll of $Q_{(\lambda: \mu)}$, so in fact this plane must lie in the quintic $\operatorname{det} L(z, y)=0$. Thus the $50 G_{5}$-translates of this plane must lie in this quintic also, so by Bezout this quintic has to be the trisecant quintic. But the map $\bar{\Theta}$ from Section 4 contracts only a curve to the point corresponding to this quintic and the claim follows.

We will show next that $B^{\prime}$ is reduced. Assume the contrary, and let $B_{\text {red }}^{\prime} \subset \operatorname{Sec} E_{(\lambda: \mu)}$ be the reduced support of $B$. To derive a contradiction we take cohomology in the exact sequence

$$
0 \rightarrow J \rightarrow \mathcal{O}_{B^{\prime}} \rightarrow \mathcal{O}_{B_{\text {red }}^{\prime}} \rightarrow 0 .
$$

Since $h^{2}\left(\mathcal{O}_{B^{\prime}}\right)=0$ it follows that $h^{2}\left(\mathcal{O}_{B_{\text {red }}^{\prime}}\right)=0$. In fact, the same argument shows that $h^{2}\left(\mathcal{O}_{Y}\right)=0$ for any subscheme $Y \subset B^{\prime}$. We now consider two cases. In any case we can apply Proposition 6.2 since $B_{\text {red }}^{\prime}$ is $H_{5}$-invariant.

If $\operatorname{deg} B_{\text {red }}^{\prime}=10$, then $B_{\text {red }}^{\prime}$ is of type $B_{\text {red }}^{\prime}=\Sigma_{(\lambda: \mu)}\left(\tau_{1}\right) \cup \Sigma_{(\lambda: \mu)}\left(\tau_{2}\right)$. So $B^{\prime}$ is a locally Cohen-Macaulay surface which is the union of a double structure $X$ on one scroll, say $\Sigma_{(\lambda: \mu)}\left(\tau_{1}\right)$, and the other scroll $\Sigma_{(\lambda: \mu)}\left(\tau_{2}\right)$. On the other side, $\operatorname{Sec} E_{(\lambda: \mu)}$ contains the unique HM-double structure on $\Sigma_{(\lambda: \mu)}\left(\tau_{1}\right)$. As a double structure contained in $\operatorname{Sec} E_{(\lambda: \mu)}$ this is uniquely determined outside the curve $E_{(\lambda: \mu)}$ since the quintic is smooth outside this curve. Since the HM-double structure is locally Cohen-Macaulay, $X$ and therefore $B^{\prime}$ must contain it as a subscheme. This contradicts the fact that $h^{2}\left(\mathcal{O}_{Y}\right)=0$ for any subscheme $Y$ of $B^{\prime}$.

If $\operatorname{deg} B_{\text {red }}^{\prime}=5$, then $B^{\prime}$ is a locally Cohen-Macaulay triple structure on a scroll $\Sigma_{(\lambda: \mu)}\left(\tau_{i}\right)$. Again, such a triple structure is unique outside the curve $E_{(\lambda: \mu)}$ since the quintic is smooth outside this curve. Therefore $B^{\prime}$ must contain the HM-double structure on the scroll, a contradiction as above.

In conclusion, $B^{\prime}$ is a $H_{5}$-invariant, reduced surface of degree 15 on $\operatorname{Sec} E_{(\lambda: \mu)}$ which is not $\iota$-invariant. Thus it is a smooth non-minimal bielliptic surface by Proposition 6.2.

At this point we want to mention one further type of $G_{5}$-invariant surfaces in $\mathbb{P}^{4}$ with $d=10$ and $\pi=6$. Namely, as we will see below, there exists a one-dimensional family of surfaces $B^{\prime \prime}$ which are a union of two $G_{5}$-invariant elliptic quintic scrolls meeting exactly along a $G_{5}$-invariant elliptic normal curve. The topological realization of the dual graph of a type III degeneration of an elliptic scroll is a Möbius band, hence the topological realization of the dual graph of a type III degeneration of two elliptic scrolls meeting exactly along an elliptic curve is a Klein bottle, i.e., two Möbius bands glued along their boundaries. This already indicates that the surfaces $B^{\prime \prime}$ should be similar, at least in terms of numerical invariants, to a minimal bielliptic surface in $\mathbb{P}^{4}$. 
On the other hand, the canonical bundle of the union of two scrolls is a 2-torsion bundle, whereas the canonical bundle of the bielliptic surfaces in $\mathbb{P}^{4}$ is 3 -torsion. This already indicates that the union of two scrolls is not a degeneration of the smooth bielliptic surfaces. In fact, we shall see in the appendix that the union of two scrolls corresponds to a smooth point in the Hilbert scheme and that every (small) deformation is again a union of two scrolls. On the other side, D. Morrison gives in [Mor1], [Mor2] a complete description of all weakly projective semistable degenerations of bielliptic surfaces with 3-torsion canonical class. Unfortunately, none of the examples of $\mathrm{H}_{5}$-invariant degenerations to be discussed in Section 9 falls in his list.

Theorem 6.7. Let $M^{\prime \prime}=\bigoplus_{k \geq 0} M_{k}^{\prime \prime}$ be the $H_{5}$-invariant graded $R$-module presented by

$$
\alpha_{1}=\left(M_{\tau_{1}}(y), M_{\tau_{2}}(y), M_{o_{E}(\lambda) \mu}(y)\right)
$$

where $\tau_{i}, i=\overline{1,2}$, are two non-trivial 2-torsion points and $o_{E_{(\lambda: \mu)}}$ is the origin on the elliptic normal curve $E_{(\lambda: \mu)} \subset \mathbb{P}^{4},(\lambda: \mu) \in \mathbb{P}_{-}^{1} \backslash \Lambda$. Then:

(i) $M^{\prime \prime}$ has Hilbert function $(5,10,10)$ and a minimal free resolution of type

$$
\begin{aligned}
& 0 \leftarrow M^{\prime \prime} \leftarrow 5 R \leftarrow 15 R(-1) \quad \begin{array}{cc}
10 R(-2) & R(-3) \\
& \quad \begin{array}{c}
\oplus \quad \\
R(-3)
\end{array} \quad \oplus
\end{array} \\
& \begin{array}{l}
\oplus \\
25 R(-4)
\end{array} 55 R(-5) \quad 40 R(-6) \leftarrow 10 R(-7) \leftarrow 0 .
\end{aligned}
$$

(ii) $S y z_{1}\left(M^{\prime \prime}(1)\right)$ is a vector bundle of rank 10 and there exists a sheaf monomorphism

$$
\varphi: 5 \mathcal{O}(-1) \oplus \Omega^{3}(3) \rightarrow \delta y z_{1}\left(M^{\prime \prime}(1)\right) .
$$

Such a morphism drops rank along the surface $B^{\prime \prime}=\Sigma_{(\lambda: \mu)}\left(\tau_{1}\right) \cup \Sigma_{(\lambda: \mu)}\left(\tau_{2}\right)$ of degree 10, and it is uniquely determined up to isomorphisms, hence $B^{\prime \prime}$ is uniquely determined by $M^{\prime \prime}$. Moreover, $B^{\prime \prime}$ is contained in precisely one quintic hypersurface, namely $\operatorname{Sec} E_{(\lambda: \mu)}$, and the ideal sheaf $\mathcal{J}_{B^{\prime \prime}}$ has a minimal free resolution of type

$$
0 \leftarrow \partial_{B^{\prime \prime}} \leftarrow \underset{25 \mathcal{O}(-6)}{\oplus} \nwarrow 55 \mathcal{O}(-7) \leftarrow 40 \mathcal{O}(-8) \leftarrow 10 \mathcal{O}(-9) \leftarrow 0 .
$$

Conversely, any union $B^{\prime \prime} \subset \mathbb{P}^{4}$ of two elliptic quintic scrolls meeting exactly along an elliptic normal curve is obtained in this way (up to coordinate transformations).

Proof. The proof of (i) and (ii) is similar to the one of Theorem 6.3. Details are left to the reader. For the converse statement let $B^{\prime \prime}=\Sigma_{(\lambda: \mu)}\left(\tau_{1}\right) \cup \Sigma_{(\lambda: \mu)}\left(\tau_{2}\right)$ be the union of the $\tau_{i}$-translation scrolls of $E_{(\lambda: \mu)}, i=1,2$. By comparing the minimal free resolutions in Lemma 4.4, (i) and (ii) we deduce easily the existence of a commutative diagram:

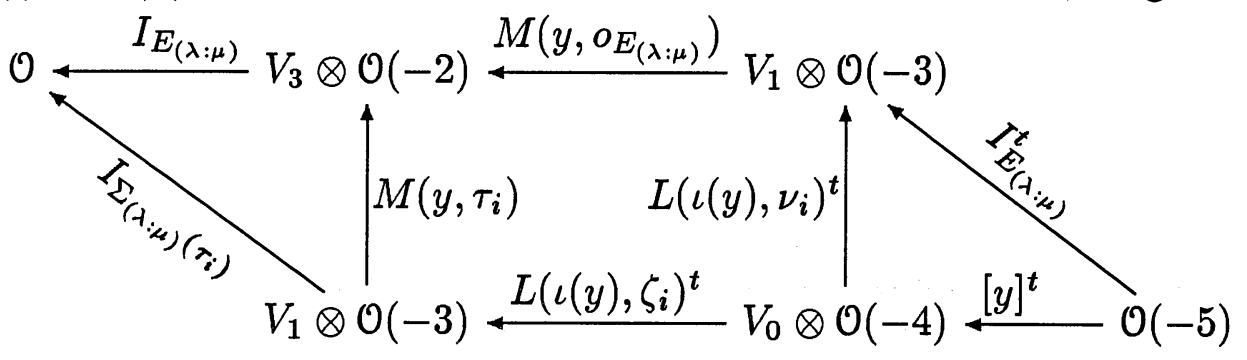


with suitably chosen $\nu_{i}$ and $\zeta_{i} \in \mathbb{P}_{-1}^{1}, i=1,2$. In particular, this provides an explicit description of the first and second order linear syzygies and of the first order quadratic syzygies of the first Hartshorne-Rao module of $B^{\prime \prime}$, from which our claim follows as in Proposition 3.7.

\section{Non-Minimal ABELIAN SURFACES OBTAINED VIA LINKAGE}

In this section we review the non-minimal abelian surfaces obtained from the minimal ones via linkage from a syzygy point of view. This also serves as a further motivation for Section 8 where we will present a new family of non-minimal abelian surfaces in $\mathbb{P}^{4}$.

Let $A=X_{s}$ be an abelian surface of degree $d=10$ and sectional genus $\pi=6$ as in Section 3. Then we can link $A$ in the complete intersection of two quintic hypersurfaces to a smooth surface $A^{\prime}$ with $d=15$ and $\pi=21$. It follows from a Bertini type argument on the blow-up of $\mathbb{P}^{4}$ in $L$ that for generic choices $A^{\prime}$ is indeed also smooth in the points of the union $L$ of the HM-lines $L_{i j}$ (Ellingsrud and Peskine, unpublished). By Proposition 2.7 (compare also [Au]) $A^{\prime}$ is a non-minimal abelian surface with the $25 \mathrm{HM}$-lines $L_{i j}$ as exceptional lines and the canonical divisor $K=\sum L_{i j}$. Moreover, $A^{\prime}$ carries a polarization of type $(2,10)[\mathrm{Au}$, Corollary 4.11], [ADHPR, Corollary 40] (compare Proposition 8.4 below). From the exact sequence

$$
0 \rightarrow \mathcal{K} \rightarrow 3 \mathcal{O}(-5) \oplus 15 \mathcal{O}(-6) \rightarrow \partial_{A} \rightarrow 0
$$

given by the syzygies of $\partial_{A}$ we deduce via linkage [PS] a resolution

$$
0 \rightarrow \mathcal{F}^{\prime}=\mathcal{O}(-5) \oplus 15 \mathcal{O}(-4) \rightarrow \mathcal{G}^{\prime}=\mathcal{K}^{\vee}(-10) \rightarrow \partial_{A^{\prime}} \rightarrow 0 .
$$

Hence $H_{*}^{2} \partial_{A^{\prime}}$ is the $\mathbb{C}$-dual module

$$
N^{\prime}=H_{*}^{2} \partial_{A^{\prime}}=H_{*}^{2} \mathcal{K}^{\vee}(-10)=\left(H_{*}^{1} \partial_{A}(5)\right)^{*}=(N(3))^{*},
$$

and $H_{*}^{1} \partial_{A^{\prime}}$ is just the vector space

$$
H_{*}^{1} \partial_{A^{\prime}}=H^{1} \partial_{A^{\prime}}(5)=\left(H^{2} \partial_{A}\right)^{*}=2 S
$$

(compare Section 3). In terms of $N^{\prime}$

$$
\mathcal{G}^{\prime}=\operatorname{ker}\left(2 \mathcal{O}(-5) \stackrel{\beta}{\leftarrow} \mathcal{S} y z_{2}\left(N^{\prime}\right)\right),
$$

where $\beta$ is induced by the minimal free resolution of $N^{\prime}$. So we may compute the equations of $A^{\prime}$ directly from $N^{\prime}$ and thus from $N$. Of course this can also be seen from the exact sequence

$$
0 \rightarrow \mathcal{E}^{\vee}(-8) \rightarrow 3 \mathcal{O}(-5) \rightarrow \partial_{A^{\prime}} \rightarrow 0
$$

obtained from (3.1) via linkage.

Proposition 7.2. J $A_{A^{\prime}}$ has syzygies of type

$$
0 \leftarrow \partial_{A^{\prime}} \leftarrow \underset{5 \mathcal{O}(-7)}{\oplus} \nwarrow 15 \mathcal{O}(-8) \leftarrow 10 \mathcal{O}(-9) \leftarrow 2 \mathcal{O}(-10) \leftarrow 0 .
$$

The quintics alone already cut out $A^{\prime}$. 
Remark 7.3. We have the following cohomology table for $\mathcal{\partial}_{A^{\prime}}$ :

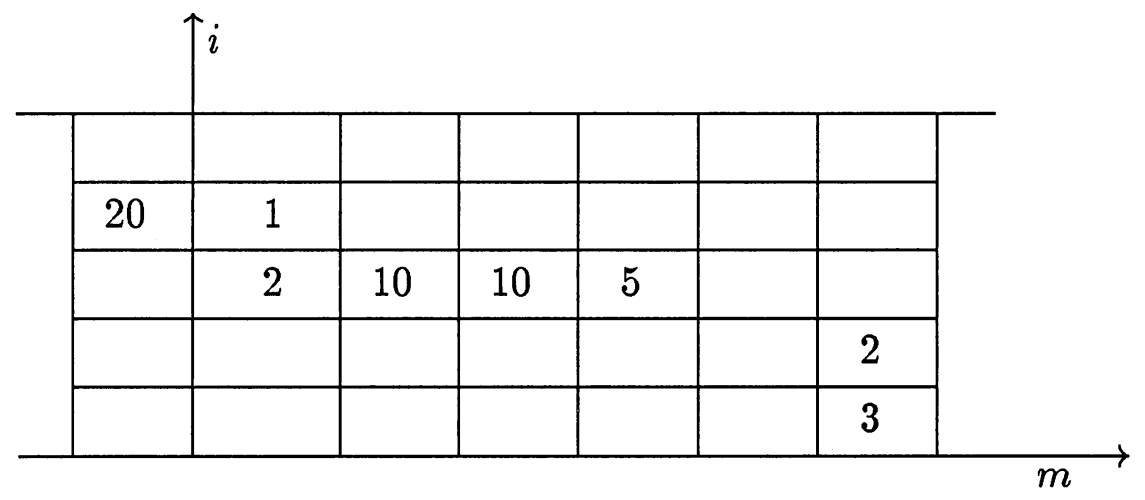

By applying Beilinson's spectral sequence to $\partial_{A^{\prime}}(3)$ we reobtain the exact sequence (7.1).

Remark 7.4. Generalized Serre correspondence [Ok2, Theorem 2.2] yields an exact sequence

$$
0 \rightarrow \mathcal{O} \rightarrow \mathcal{E}^{\prime}(3) \rightarrow \mathcal{J}_{A^{\prime}}(5) \rightarrow 0,
$$

where $\mathcal{E}^{\prime}$ is a stable rank 2 reflexive sheaf with Chern classes $c_{1}=-1, c_{2}=9, c_{3}=25$ and $c_{4}=50$. Since

$$
\operatorname{dim} \operatorname{Ext}^{1}\left(\partial_{A^{\prime}}(5), \mathcal{O}\right)=p_{g}\left(A^{\prime}\right)=1,
$$

this extension is uniquely determined. So it coincides with the one given by Beilinson's spectral sequence applied to $\partial_{A^{\prime}}(4)$. ¿ From (7.1) we can derive an alternative description: $\mathcal{E}^{\prime}(-2)$ is the cokernel

$$
0 \rightarrow 15 \mathcal{O}(-4) \stackrel{\gamma}{\rightarrow} \mathcal{G}^{\prime} \rightarrow \mathcal{E}^{\prime}(-2) \rightarrow 0,
$$

where $\gamma$ is given by the syzygies of $\mathcal{G}^{\prime}$. So $\mathcal{E}^{\prime}$ depends on $N^{\prime}$ only and plays the role of the HM-bundle for the linked surfaces.

\section{A NEW FAMILY OF NON-MINIMAL ABELIAN SURFACES}

In this section we present the construction of a new family of non-minimal abelian surfaces of degree 15 and sectional genus 21 in $\mathbb{P}^{4}$. This construction and Proposition 8.2 are taken from [Po, Section 7]. For some of our results we need to rely on a computation using [Mac].

Let $\Pi=<p_{1}, p_{2}, p_{3}>$ be a plane as in (5.1), (iii) and Proposition 5.6 with $p_{1}=a=\left(a_{0}\right.$ : $\left.a_{1}: a_{2}: a_{2}: a_{1}\right) \in \mathbb{P}_{+}^{2} \backslash \Delta, p_{2}=(0: 1: 0: 0:-1)$ and $p_{3}=(0: 0: 1:-1: 0)$, and let $M$ be the $G_{5}$-invariant graded $R$-module with representing plane $\Pi$. Then $M$ is artinian by Proposition 5.6. By construction $M_{0} \cong V_{3}$ and $M_{1} \cong 2 V_{1}$ as $G_{5}$-modules. We will use the double complex of the Koszul cohomology to compute $M_{k}$ for $k \geq 2$. The kernel of the vertical differential $4 V_{0} \oplus 6 V_{0}^{\#} \rightarrow M_{1} \otimes V_{2} \cong 6 V_{0} \oplus 4 V_{0}^{\#}$ contains $2 V_{0}^{\#}$, and in fact equality holds by Proposition 5.8. Therefore $M_{2} \cong 2 V_{0}^{\#}$. $M$ has no second order linear syzygies by Proposition 5.10 and it has exactly 2 genuine first order quadratic syzygies by Proposition 5.11. Thus a similar argument as above shows that $M_{3} \cong 2 I$. Moreover, $M$ has no second order quadratic syzygies by Proposition 5.12.

In the sequel we will claim correctness of our results only for a general point $p_{1}=a \epsilon$ $\mathbb{P}_{+}^{2} \backslash \Delta$. In fact, the module $M$ with representing plane $\Pi$ will lead to a smooth surface only if we choose $p_{1}$ general enough (compare Section 9). So it will be sufficient for our purposes to 
check in an example that some Zariski open conditions are non empty. E.g., we can check in an example, say $p_{1}=(1: 0: 0: 0: 0)$, that $M_{k}=0$ for $k \geq 4$. Therefore, for generic choices, $M$ has Hilbert function $(5,10,10,2)$ as in the case of the first Hartshorne-Rao module of the HM-surfaces (compare Proposition 3.2). But this time the minimal free resolution of $N_{a}=(M)^{\vee}(3)$ is of the form

$$
\begin{aligned}
& 0 \leftarrow N_{a} \leftarrow 2 R \\
& \begin{array}{rc}
\nwarrow 20 R(-2) \leftarrow 35 R(-3) & 15 R(-4) \\
& \begin{array}{c}
\oplus \\
2 R(-5)
\end{array}
\end{array} \\
& \oplus \\
& 10 R(-6) \quad 15 R(-7) \leftarrow 5 R(-8) \leftarrow 0 \text {. }
\end{aligned}
$$

Comparing with (7.1) we define

$$
\mathcal{F}=\mathcal{O}(-5) \oplus 15 \mathcal{O}(-4), \quad \mathcal{S}_{a}=S_{y} z_{2}\left(N_{a}\right) .
$$

As one can check with [Mac] in an example the generic $\varphi \in \operatorname{Hom}\left(\mathcal{F}, \mathcal{G}_{a}\right)$ gives rise to a smooth surface $A_{a} \subset \mathbb{P}^{4}$ with $d=15, \pi=21$ and $\chi=0$. By construction $\partial_{A_{a}}$ has a minimal free resolution of type

$$
0 \leftarrow \partial_{A_{a}} \leftarrow \underset{10 \mathcal{O}(-6)}{\bigoplus} \nwarrow 15 \mathcal{O}(-7) \leftarrow 5 \mathcal{O}(-8) \leftarrow 0
$$

and the cohomology table

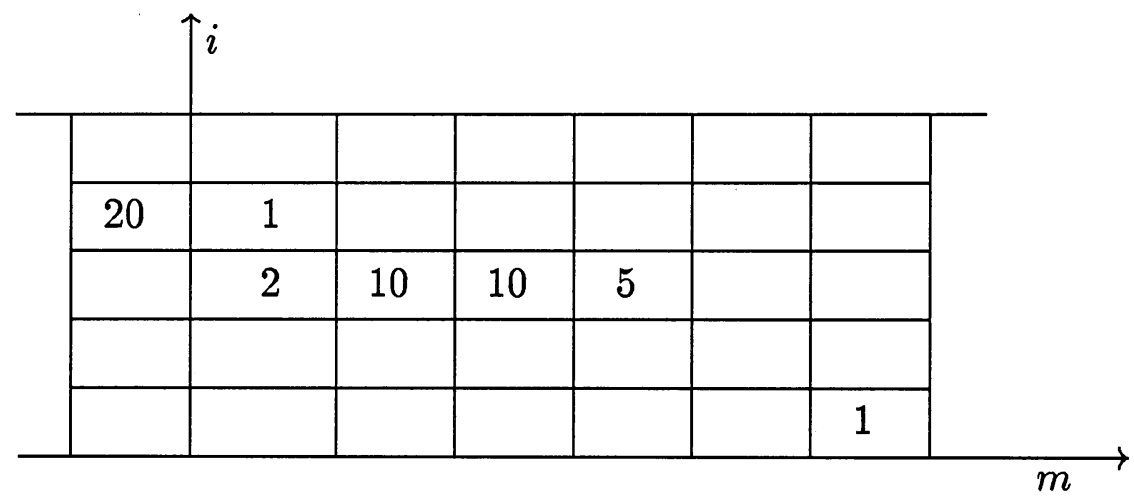

Hence by Proposition 2.7 $A_{a}$ is a non-minimal abelian surface with 25 exceptional lines. Generalized Serre correspondence yields an exact sequence

$$
0 \rightarrow \mathcal{O} \rightarrow \mathcal{E}_{a}(3) \rightarrow \partial_{A_{a}}(5) \rightarrow 0
$$

where $\mathcal{E}_{a}$ is a stable rank 2 reflexive sheaf on $\mathbb{P}^{4}$ with Chern classes $c_{1}=-1, c_{2}=9$, $c_{3}=25$ and $c_{4}=50$. As in Remark 7.4 one sees that (8.1) can also be obtained by applying Beilinson's spectral sequence to $\mathcal{J}_{A_{a}}(4)$, and hence deduce that $\mathcal{E}_{a}$ is the cokernel

$$
0 \rightarrow 15 \mathcal{O}(-4) \stackrel{\gamma}{\rightarrow} \mathcal{G}_{a} \rightarrow \mathcal{E}_{a}(-2) \rightarrow 0
$$

where $\gamma$ is induced by the syzygies. In any case $H^{0}\left(\mathcal{E}_{a}(3)\right)$ has dimension 2 . 
Proposition 8.2. Fix a general point $p_{1}=a$ as above and a basis $s_{1}, s_{2}$ of $H^{0}\left(\mathcal{E}_{a}(3)\right)$. Then there exists a pencil

$$
A_{(\lambda: \mu)}=\left\{\lambda s_{1}+\mu s_{2}=0\right\}
$$

of $G_{5}$-invariant, smooth, non-minimal abelian surfaces with $d=15, \pi=21$, all lying on the unique quintic hypersurface

$$
U=\left\{s_{1} \wedge s_{2}=0\right\} \subset \mathbb{P}^{4}
$$

Moreover

$$
\operatorname{Sing}\left(\mathcal{E}_{a}\right)=\bigcup E_{i j}
$$

consists of the 25 lines $E_{i j}$ obtained as the $H_{5}$-translates of the line

$$
E_{00}=\left\{y_{1}-y_{4}=y_{2}-y_{3}=a_{0} y_{0}+2 a_{1} y_{1}+2 a_{2} y_{2}=0\right\} \subset \mathbb{P}_{+}^{2} .
$$

These lines are in the base locus of the pencil and hence are the exceptional lines of the smooth members in the pencil.

Proof. $\mathcal{E}_{a}$ has the syzygies

$$
0 \leftarrow \mathcal{E}_{a} \leftarrow \underset{10 \mathcal{O}(-4) .}{\bigoplus} \stackrel{\beta=\left(\alpha_{22}^{t}, \alpha_{21}^{t}\right)}{\longleftarrow} 15 \mathcal{O}(-5) \stackrel{\alpha_{1}^{t}}{\longleftarrow} 5 \mathcal{O}(-6) \leftarrow 0,
$$

where the maps are given by the minimal free resolution of $N_{a}$. Recall that

$$
\alpha_{21}=\left(\begin{array}{ll}
L_{q_{11}}(y) & L_{q_{12}}(y) \\
L_{q_{21}}(y) & L_{q_{22}}(y) \\
L_{q_{31}}(y) & L_{q_{32}}(y)
\end{array}\right)
$$

with $q_{i j}, 1 \leq i \leq 3,1 \leq j \leq 2$, as in Proposition 5.8. $\alpha_{22}$ is given by the 2 genuine first order quadratic syzygies of $\alpha_{1}$ which we have to choose among all first order quadratic syzygies corresponding to the trivial character of $H_{5}$ as in the proof of Proposition 5.11. There are 4 such syzygies which we collect in the matrix

$$
S=\left(\begin{array}{cccc}
0 & 0 & 0 & 0 \\
\left(y_{i+2} y_{i+3}\right)_{i \in \mathbb{Z}_{5}} & 0 & \left(y_{i+1} y_{i+4}\right)_{i \in \mathbb{Z}_{5}} & \left(y_{i}^{2}\right)_{i \in \mathbb{Z}_{5}} \\
0 & \left(y_{i+1} y_{i+4}\right)_{i \in \mathbb{Z}_{5}} & \left(y_{i}^{2}\right)_{i \in \mathbb{Z}_{5}} & -\left(y_{i+2} y_{i+3}\right)_{i \in \mathbb{Z}_{5}}
\end{array}\right)
$$

Modulo the first order linear syzygies we have the relations

$$
S \cdot\left(\begin{array}{cc}
a_{1} & a_{0} \\
-a_{0} & a_{2} \\
0 & -a_{1} \\
-a_{2} & 0
\end{array}\right)=0
$$

which tell us how to choose $\alpha_{22}$ depending on $a$. For the general point $p_{1}=a$ one can check that $\beta$ drops rank on $E_{00}$ and thus also on all the $H_{5}$-translates $E_{i j}$. Hence $\bigcup E_{i j} \subset \operatorname{Sing}\left(\mathcal{E}_{a}\right)$ and equality follows from

$$
\operatorname{deg} \operatorname{Sing}\left(\mathcal{E}_{a}\right)=c_{3}(\mathcal{E})=25 .
$$

That $\bigcup E_{i j}$ is contained in the base locus is clear from generalized Serre correspondence. 
Remark 8.3. (i) One can show that in fact $\mathcal{E}_{a}(3)$ is globally generated outside $\bigcup E_{i j}$ and that $\bigcup E_{i j}$ is precisely the base locus of the pencil $A_{(\lambda: \mu)}$.

(ii) The quintic hypersurface $U$ is interesting in its own and has a rich geometry. For details compare [Po, Section 7] and [GP2].

Proposition 8.4. (i) If $A_{a}$ is a new abelian surface, then $H$ is of type (1,20).

(ii) If $A^{\prime}$ is a linked abelian surface as in Section 7, then $H$ is of type (2,10).

Proof. (i) One can check using [Mac] that $A_{a}$ intersects $\mathbb{P}_{-}^{1}$ in 3 points and $\mathbb{P}_{+}^{2}$ in the line $E_{00}$ and 12 further points. Choose any of these 12 points as the origin of $A_{a}$ (strictly speaking of the minimal model $A_{a}^{\mathrm{min}}$ of $A_{a}$, of course). The involution $\iota$ leaves the union of the 25 lines $E_{i j}$ invariant and descends to an involution $\iota$ on $A_{a}^{\min }$. $\iota$ has exactly 16 fixed points on $A_{a}^{\mathrm{min}}$, namely the images of the points where $A_{a}$ intersects $\mathbb{P}_{-}^{1}$ and $\mathbb{P}_{+}^{2}$ resp., and the point which arises from blowing down the line which is contained in the intersection of $A_{a}$ and $\mathbb{P}_{+}^{2}$. Moreover, $\iota$ induces an involution on $\mathcal{L}=\mathcal{O}_{A_{a}^{\min }}(H)$, i.e., $\iota$ is an involution of the polarized abelian surface $\left(A_{a}^{\min }, \mathcal{L}\right)$ and $\mathcal{L}$ is a symmetric line bundle, i.e., $\iota^{*} \mathcal{L}=\mathcal{L}$. Since $\iota$ has exactly 16 fixed points it must be the involution $x \mapsto-x$ on $A_{a}^{\text {min }}$. This follows from the Lefschetz fixed point formula (see e.g. [BL]). The involution $\iota$ has 12 even 2-torsion points (they come from the 12 isolated points of $\left.\left(\mathbb{P}^{2}\right)^{+} \cap A_{a}\right)$ and 4 odd 2-torsion points (these come from $\left(\mathbb{P}^{1}\right)^{-} \cap A_{a}$ and the invariant line that was blown down). Hence by [LB, Proposition 4.7.5] the polarization given by $\mathcal{L}$ must be of type $(1,20)$.

(ii) In this case we get by the same argument that there are 16 even 2-torsion points and no odd 2-torsion points. Again by [LB, Proposition 4.7.5] the polarization must be the square of a primitive polarization, i.e., it must be of type $(2,10)$.

\section{DEGENERATIONS OF ABELIAN AND BIELLIPTIC SURFACES.}

In this section we describe several types of $\mathrm{H}_{5}$-invariant degenerations of abelian and bielliptic surfaces of degree 10 and 15. The degenerations of the smooth HM-surfaces are already completely classified and very well understood (cf. [HM], [BHM2], [HV], [BM], [Hu2]). Therefore we will focus in the sequel only on degenerations of the other abelian and bielliptic surfaces described in this paper. Our results are in the spirit of those in [BHM2], but we do not aim at complete lists here. Nevertheless our results underline the fact that the Hilbert scheme is connected [Ha].

In order to construct degenerations we specialize the representing planes of the modules which give rise to the smooth surfaces. In (5.1), (ii'), (iv) and (v) these planes are spanned by torsion points on an elliptic normal curve. Therefore we first need to understand the limits of such points on a singular fibre of the Shioda modular surface $S(5)$. We recall that all such fibres are mapped to pentagons in $\mathbb{P}^{4}$ via the normalization map described in Proposition 4.2. For the degenerations we look at the limit points on the standard pentagons $E_{(0: 1)}$ and $E_{(1: 0)}$ with vertices $e_{0}, \ldots, e_{4}$ only. On $S(5)$ we have the following:

Lemma 9.1. Let $\mathcal{D}, X$, and $\mathcal{T}$ resp. be the 3-, 8-, and 24-section resp. of the Shioda modular surface $S(5)$ defined by the torsion points of order 2, 3, and 6 resp. on the smooth fibres. Furthermore, let $O$ denote the zero section, and let $\Gamma$ be a singular fibre, say with vertices $e_{0}, \ldots, e_{4}$, such that the involution $\iota$ operates by $e_{i} \rightarrow e_{-i}$. If the edges of $\Gamma$ are $\overline{e_{i+2} e_{i+3}}, i \in \mathbb{Z}_{5}$, then the following is true:

(i) $O$ intersects $\Gamma$ in the fixed point of $\iota$ on $\overline{e_{2} e_{3}}$ which corresponds to (1:-1). 
(ii) $\mathcal{D}$ passes transversely through the $\iota$-invariant point on $\overline{e_{2} e_{3}}$ which corresponds to (1:1), and it passes with multiplicity 2 through $e_{0}$.

(iii) $X$ passes with multiplicity 3 through $e_{1}$ and $e_{4}$ and intersects transversely $\overline{e_{2} e_{3}}$ in 2 points corresponding to the 2 primitive roots of -1 of order 3.

(iv) $\mathcal{T}$ passes with multiplicity 4 through $e_{0}$, with multiplicity 3 through $e_{1}$ and $e_{4}$, with multiplicity 6 through $e_{2}$ and $e_{3}$, and intersects transversely $\overline{e_{2} e_{3}}$ in 2 points corresponding to the 2 primitive roots of unity of order 3.

The analogous results hold for the pentagon with edges $\overline{e_{i+1} e_{i+4}}, i \in \mathbb{Z}_{5}$.

Proof. (i) and (ii) are well-known, see [Sh], [Na] or [HKW, Proposition 2.33] for a proof and comments. For (iii) and (iv) one can proceed as in the proof of [HKW, Proposition 2.33], along with a careful book-keeping of the various glueings in the toroidal compactification. Another method is to make these computations directly on a projective model of $S(5)$, namely in our case on $S_{15} \subset \mathbb{P}^{4}$ with $\Gamma=E_{(0: 1)}$. In this setting (iii) follows directly from Proposition 4.13. In fact, on $\overline{e_{2} e_{3}}$ the limits of the proper 3 -torsion points are defined by the equation $y_{2}^{2}-y_{2} y_{3}+y_{3}^{2}=0$. For (iv) one uses the fact that the octic hypersurface $w_{0}^{2}+\left(w_{1}+w_{4}\right)\left(w_{2}+w_{3}\right)$, where $w_{2 i}=y_{i}\left(y_{i+2} y_{i+4}^{2}-y_{i+1}^{2} y_{i+3}\right), i \in \mathbb{Z}_{5}$, meets each smooth fiber of $S_{15}$ in 6-torsion points only by Proposition 4.19. Thus, for instance, on $\overline{e_{2} e_{3}}$ the limits of the proper 6 -torsion points are defined by the equation $y_{2}^{6} y_{3}^{6}\left(y_{2}^{2}+y_{2} y_{3}+y_{3}^{2}\right)=0$. The proof of the other assertions in (iv) is similar.

We are now ready to study degenerations of our surfaces. Again a first question will be whether our limit planes represent artinian modules with the expected number of syzygies, i.e., with the same graded Betti numbers as the general members of our families. The planes contained in coordinate hyperplanes are not of interest for us:

Remark 9.2. Any plane $\Pi$ inside a coordinate hyperplane $\left\{x_{i}=0\right\}$ gives rise to a nonartinian module by Lemma 5.3, since $\Pi$ is contained in the kernel of $N\left(e_{i}, e_{i}\right)$.

For the minimal bielliptic surfaces of degree 10 the first Hartshorne-Rao module of the surface has a representing plane of type $\Pi=<\tau_{1}, \tau_{2}, \tau_{3}+\rho>$, where $\tau_{i}, i=\overline{1,3}$ are the non-trivial 2 -torsion points and $\rho$ is a non-trivial 3 -torsion point on a $G_{5}$-invariant elliptic normal curve in $\mathbb{P}^{4}$. Remark 9.2 rules out most of the possible limit planes. We may, however, consider the case where the points $\tau_{1}$ and $\tau_{2}$ come together at $e_{0}$, while $\tau_{3}+\rho$ goes to one of the two possible limit points on $\overline{e_{2} e_{3}}$ (in the case of $E_{(0: 1)}$ ) or $\overline{e_{1} e_{4}}$ (in the case of $\left.E_{(1: 0)}\right)$. There are two branches of the Brings curve $B$ through $e_{0}$ with tangents $\left\{y_{2}=y_{3}=y_{1}-y_{4}=0\right\}$ or $\left\{y_{2}-y_{3}=y_{1}=y_{4}=0\right\}$. This gives rise to four limit planes which are spanned by $\left\{y_{2}=y_{3}=y_{1}-y_{4}=0\right\}$ and one of the two points $\left(0: 0: \theta_{k}: 1: 0\right) \in \overline{e_{2} e_{3}}$ or by $\left\{y_{2}-y_{3}=y_{1}=y_{4}=0\right\}$ and one of the two points $\left(0: \theta_{k}: 0: 0: 1\right) \in \overline{e_{1} e_{4}}$, where $\theta_{1}$ and $\theta_{2}$ are two different primitive third roots of unity. One checks through a direct computation that these limit planes define indeed four artinian graded modules with the Hilbert function $(5,10,10)$ and with the expected syzygies. Moreover, a determinantal construction as in Proposition 3.7 and Theorem 6.3 leads to four locally Cohen-Macaulay surfaces $Y_{\left(\theta_{k}: 1\right)}$ or $Z_{\left(\theta_{k}: 1\right)}$ in $\mathbb{P}^{4}$ with the same numerical invariants as a smooth minimal bielliptic surface of degree 10.

We will describe the equations of these degenerations. In fact, $Y_{\left(\theta_{k}: 1\right)}$ and $Z_{\left(\theta_{k}: 1\right)}$ can be seen as special points of other components of the Hilbert scheme. Namely, consider the surfaces

$$
Y_{(\lambda: \mu)}=\bigcup_{i \in \mathbb{Z}_{5}} Y_{i}^{(\lambda: \mu)} \text { and } Z_{(\lambda: \mu)}=\bigcup_{i \in \mathbb{Z}_{5}} Z_{i}^{(\lambda: \mu)}
$$


where each $Y_{i}^{(\lambda: \mu)}$ and $Z_{i}^{(\lambda: \mu)}$ is a double structure (only generically Cohen-Macaulay) on a plane:

$$
Y_{i}^{(\lambda: \mu)}=\left\{y_{i}^{2}=y_{i+2}^{2}=y_{i} y_{i+2}=\lambda y_{i+2} y_{i+3}^{2}+\mu y_{i} y_{i+4}^{2}=0\right\}
$$

and

$$
Z_{i}^{(\lambda: \mu)}=\left\{y_{i}^{2}=y_{i+1}^{2}=y_{i} y_{i+1}=\lambda y_{i+1} y_{i+4}^{2}+\mu y_{i} y_{i+2}^{2}=0\right\}
$$

The reduced support of $Y_{(\lambda: \mu)}$ or $Z_{(\lambda: \mu)}$ is the union of 5 planes, which in turn is a degeneration of an elliptic quintic scroll in $\mathbb{P}^{4}$. Therefore, in a way, these surfaces are double structures on a degenerate elliptic quintic scroll. $Y_{(1: 1)}$ and $Z_{(1: 1)}$ are HM-surfaces and lie on three quintics, but for the general point $(\lambda: \mu)$ these surfaces lie on the same unique degenerate quintic $\left\{\gamma_{0}=y_{0} y_{1} y_{2} y_{3} y_{4}=0\right\}$. Correspondingly, the plane which is spanned by the tangent $\left\{y_{2}=y_{3}=y_{1}-y_{4}=0\right\}$ and a point $(0: 0: \lambda: \mu: 0) \in \overline{e_{2} e_{3}}$ or the tangent $\left\{y_{2}-y_{3}=y_{1}=y_{4}=0\right\}$ and a point $(0: \lambda: 0: 0: \mu) \in \overline{e_{1} e_{4}}$ is $\mathbb{P}_{+}^{2}$ for $(\lambda: \mu)=(1: 1)$, whereas for the general point $(\lambda: \mu)$ this plane represents an artinian module with the same number of syzygies as for the minimal bielliptics.

Now recall that the first Hartshorne-Rao module of the union of two $G_{5}$-invariant elliptic quintic scrolls as in Theorem 6.7 has a representing plane of type $\Pi=\left\langle\tau_{1}, \tau_{2}, o\right\rangle$, where $\tau_{i}, i=\overline{1,2}$, are two non-trivial 2 -torsion points and $o$ is the origin of a $G_{5}$-invariant elliptic normal curve in $\mathbb{P}^{4}$. Therefore we can specialize as above such that $\tau_{1}$ and $\tau_{2}$ come together to the vertex $e_{0}$, while $o$ goes to the point $(0: 0: 1:-1: 0) \in \overline{e_{2} e_{3}}$ or $(0: 1: 0: 0-1) \in \overline{e_{1} e_{4}}$. Thus $Y_{(1:-1)}$ and $Z_{(1:-1)}$ are degenerations of the union of two elliptic quintic scrolls as in Proposition 6.7.

Next we study the non-minimal bielliptic surfaces of degree 15 as in Theorem 6.6. The dual of the second Hartshorne-Rao module of such a surface has a representing plane of type $\Pi=\left\langle\tau_{1}+\rho, \tau_{2}+\rho, \tau_{3}+\rho>\right.$, where $\tau_{i}, i=\overline{1,3}$ are the three non-trivial 2 -torsion points and $\rho$ is a non-trivial 3 -torsion point of a $G_{5}$-invariant elliptic normal curve in $\mathbb{P}^{4}$. As in the case of the minimal bielliptic surfaces Remark 9.2 rules out most of the possible limit planes. Some limit planes however give rise to artinian modules. Let $\tau_{1}+\rho$ and $\tau_{2}+\rho$ come together at the vertex $e_{0}$ while the third point $\tau_{3}+\rho$ goes to one of the two possible limit points on $\overline{e_{2} e_{3}}$ (in the case of $\left.E_{(0: 1)}\right)$ or $\overline{e_{1} e_{4}}$ (in the case of $\left.E_{(1: 0)}\right)$. The image in $\mathbb{P}^{4}$ of the curve $\mathcal{T}$ of points of order 6 has four branches through $e_{0}$ with tangents spanned by $e_{0}$ and one of the four points corresponding to primitive roots of unity on the lines $\overline{e_{2} e_{3}}$ and $\overline{e_{1} e_{4}}$. This gives rise to four limit planes containing $e_{0}$ and meeting $\overline{e_{2} e_{3}}$ and $\overline{e_{1} e_{4}}$ transversally at points of order 6 . The corresponding modules are artinian and give rise to four reduced but reducible surfaces. These are the union of five cubic surfaces, namely

$$
\left\{y_{i}=\theta_{k} y_{i+1}^{2} y_{i+3}+y_{i+2} y_{i+4}^{2}=0\right\}, \quad i \in \mathbb{Z}_{5},
$$

or

$$
\left\{y_{i}=\theta_{k} y_{i+3}^{2} y_{i+4}+y_{i+1} y_{i+2}^{2}=0\right\}, \quad i \in \mathbb{Z}_{5},
$$

for $k=1,2$, where again $\theta_{1}$ and $\theta_{2}$ are two different primitive third roots of unity. In each case the first five cubic surfaces glue along the pentagon $E_{(0: 1)}$, while the last five cubic surfaces glue along the pentagon $E_{(1: 0)}$. As in the minimal bielliptic case these degenerations belong to pencils of surfaces, where this time the surface corresponding to $(\lambda: \mu) \in \mathbb{P}^{1}$ has the five components

$$
\left\{y_{i}=\lambda y_{i+1}^{2} y_{i+3}+\mu y_{i+2} y_{i+4}^{2}=0\right\}, \quad i \in \mathbb{Z}_{5},
$$


or

$$
\left\{y_{i}=\lambda y_{i+3}^{2} y_{i+4}+\mu y_{i+1} y_{i+2}^{2}=0\right\}, \quad i \in \mathbb{Z}_{5} .
$$

For $(\lambda: \mu)=(1: 1)$ these surfaces are $(5,5)$-linked to HM-surfaces and lie on on three quintics, but for the general point $(\lambda: \mu)$ these surfaces lie on the same unique degenerate quintic $\left\{\gamma_{0}=y_{0} y_{1} y_{2} y_{3} y_{4}=0\right\}$. Correspondingly, the plane spanned by $e_{0}, \lambda e_{4}+\mu e_{1}$ and $\lambda e_{2}+\mu e_{3}$ or $e_{0}, \lambda e_{1}+\mu e_{4}$ and $\lambda e_{2}+\mu e_{3}$ is $\mathbb{P}_{+}^{2}$ for $(\lambda: \mu)=(1: 1)$ while for the general point $(\lambda: \mu)$ this plane represents a module with the same number of syzygies as for the non-minimal bielliptic surfaces of degree 15. Recall from [Po] that there is a pencil of degenerations of the linked abelian surfaces, such that the surface corresponding to $(\lambda: \mu) \in \mathbb{P}^{1}$ has the five components

$$
\left\{y_{i}=\lambda\left(y_{i+1}^{2} y_{i+3}+y_{i+2} y_{i+4}^{2}\right)+\mu\left(y_{i+1} y_{i+2}^{2}+y_{i+3}^{2} y_{i+4}\right)=0\right\}, \quad i \in \mathbb{Z}_{5} .
$$

Now we will discuss briefly some degenerations of the new non-minimal abelian surfaces of degree 15. The following results are taken from [Po, Section 7].

We recall that for these surfaces the dual of the second Hartshorne-Rao module has a representing plane spanned by $\mathbb{P}_{-}^{1}$ and a general point $a \in \mathbb{P}_{+}^{2}$. We have seen in Proposition 8.2 that such a module gives rise to a pencil $A_{(\lambda: \mu)}$ of non-minimal abelian surfaces, all lying on the same unique quintic hypersurface. It is shown in [Po, Lemma 7.6] that this quintic is uniquely determined as the $H_{5}$-invariant quintic containing the 25 exceptional lines of the smooth members of the pencil, i.e., the 25 lines $E_{i j}$ obtained as the $H_{5}$-translates of the line

$$
E_{00}=\left\{y_{1}-y_{4}=y_{2}-y_{3}=a_{0} y_{0}+2 a_{1} y_{1}+2 a_{2} y_{2}=0\right\} \subset \mathbb{P}_{+}^{2}
$$

(compare again Proposition 8.2). To obtain degenerations we can either specialize the parameter point $a$ or look at special members of the pencil $A_{(\lambda: \mu)}$.

A first type of degenerations corresponds to the choice of the parameter point $a \in \mathbb{P}_{+}^{2}$ as one of the nodes of the Brings curve, say $a=(1: 0: 0: 0: 0)$. These degenerations are singular surfaces $X_{(\lambda: \mu)} \subset \mathbb{P}^{4},(\lambda: \mu) \in \mathbb{P}^{1}$, which all lie on the degenerate quintic hypersurface $\left\{\gamma_{0}=y_{0} y_{1} y_{2} y_{3} y_{4}=0\right\}$. A straightforward but tedious computation shows that

$$
X_{(\lambda: \mu)}=\bigcup_{i \in \mathbb{Z}_{5}} X_{i}^{(\lambda: \mu)}
$$

where the $X_{i}^{(\lambda: \mu)}$ are the cubic surfaces defined by

$$
X_{i}^{(\lambda: \mu)}=\left\{y_{i}=\lambda\left(y_{i+1}^{2} y_{i+3}-y_{i+2} y_{i+4}^{2}\right)+\mu\left(y_{i+1} y_{i+2}^{2}-y_{i+3}^{2} y_{i+4}\right)=0\right\} .
$$

We fix now a general $(\lambda: \mu) \in \mathbb{P}^{1}$ and write $X_{i}=X_{i}^{(\lambda: \mu)}$.

Proposition 9.3. (i) $X_{i}$ is a smooth Del Pezzo cubic surface in the hyperplane $H_{i}=$ $\left\{y_{i}=0\right\}$. Moreover, it is invariant under the action of $\tau$, whereas $\sigma\left(X_{i}\right)=X_{i-1}, i \in \mathbb{Z}_{5}$.

(ii) Two surfaces $X_{i}$ and $X_{j}, i \neq j$, meet along a smooth conic and a point outside the conic. There are altogether five such points, namely the vertices $e_{i}, i \in \mathbb{Z}_{5}$, of the complete pentagon $E_{(0: 1)} \cup E_{(1: 0)}$. Through each point $e_{i}$ there pass exactly four such Del Pezzo surfaces. 
(iii) Write $E_{(0: 1)}=\bigcup_{i \in \mathbb{Z}_{5}} L_{i}$ with $L_{i}=\left\{y_{i+2}=y_{i+3}=y_{i+4}=0\right\}, i \in \mathbb{Z}_{5}$, and $E_{(1: 0)}=$ $\cup_{i \in \mathbb{Z}_{5}} L^{\prime}{ }_{i}$ with $L^{\prime}{ }_{i}=\left\{y_{i+1}=y_{i+3}=y_{i+4}=0\right\}, i \in \mathbb{Z}_{5}$. Then

$$
X \cap H_{i}=X_{i} \cup L^{\prime}{ }_{i+1} \cup L_{i+2} \cup L_{i+3} \cup L^{\prime}{ }_{i+4} .
$$

Furthermore, these four lines are exceptional on all $X_{j}$ with $j \in \mathbb{Z}_{5} \backslash\{i\}$.

Proof. The claims follow by a straightforward computation using the explicit equations of $X_{i}$.

Observe now that for this choice of the parameter point the exceptional lines of the degree 15 non-minimal abelian surfaces degenerate to $\tilde{E}_{i j}=\sigma^{i} \tau^{j} \tilde{E}_{00}, i, j \in \mathbb{Z}_{5}$, where $\tilde{E}_{00}=\left\{y_{0}=y_{1}-y_{4}=y_{2}-y_{3}=0\right\}$. It is easily checked that $\tilde{E}_{i 0}, \tilde{E}_{i 1}, \tilde{E}_{i 2}, \tilde{E}_{i 3}$ and $\tilde{E}_{i 4}$ are (-1)-curves on the Del Pezzo surface $X_{i}$. As a consequence we obtain the following geometric characterization of our configuration:

Proposition 9.4. For each $i \in \mathbb{Z}_{5}$ the lines $\tilde{E}_{i 0}, \tilde{E}_{i 1}, \tilde{E}_{i 2}, \tilde{E}_{i 3}, \tilde{E}_{i 4}$ and $L_{i+3}, L_{i+1}^{\prime}$ can be completed to a Schläfi double six configuration of lines in the hyperplane $H_{i}$, which in turn determines the Del Pezzo surface $X_{i}$ as the unique cubic surface in $H_{i}$ containing the given double six.

Proof. The intersection patterns are clear from the explicit description of the configuration, and thus the claim follows from $[\mathrm{HCV}, \S 25]$.

We will finally mention some degenerations, which we have checked in an explicit example using [Mac]. For some of them see [Po] and [GP2] for more details.

First of all each general pencil $A_{(\lambda: \mu)}$ of non-minimal abelian surfaces as above contains degenerations which are ruled surfaces $\mathbb{P}\left(\mathcal{O}_{E}+\mathcal{L}\right)$, where $E$ is an elliptic curve and $\mathcal{L} \in$ $\operatorname{Pic}^{0}(E)$, mapped to $\mathbb{P}^{4}$ either by $H \equiv C_{0}+20 f_{o}-\sum_{i, j \in \mathbb{Z}^{5}} E_{i j}$ or by $H \equiv 4 C_{0}+5 f_{o}-$ $\sum_{i, j \in \mathbb{Z}^{5}} E_{i j}$, where in both cases the 25 exceptional lines form an $H_{5}$-orbit. In the first case the image in $\mathbb{P}^{4}$ is a scroll of degree 15 with singularities along the image of $C_{0}$, which is a curve of degree 20, arithmetic genus 26 and with 25 nodes (the images of the points where the curves $f_{o}-E_{i j}$ meet $C_{0}$ ). In the second case the image is a ruled surface of degree 15, with singularities along the image of $C_{0}$, which is an elliptic curve of degree 5 .

Another class of degenerations corresponds to the choice of the parameter point $a$ as a general point on the Brings curve. These are conic bundles $\mathbb{P}\left(\mathcal{O}_{E}+\mathcal{L}\right)$, with $E$ and $\mathcal{L}$ as above, mapped to $\mathbb{P}^{4}$ by $H \equiv 2 C_{0}+10 f_{o}-\sum_{i, j \in \mathbb{Z}^{5}} E_{i j}$.

Finally, for a choice of the parameter point $a$ as a general point on the modular conic $C_{+} \subset \mathbb{P}_{+}^{2}$, we obtain a pencil of surfaces $X_{(\lambda: \mu)}$, which has an elliptic quintic scroll as base component. Furthermore, $X_{(\lambda: \mu)}$ contains residual to the elliptic scroll a surface $T_{(\lambda: \mu)}$ of degree 10 and sectional genus 6 . For general $(\lambda: \mu) \in \mathbb{P}^{1}$ the surface $T_{(\lambda: \mu)}$ is a smooth minimal abelian surface isogenous to a product, and meeting the base elliptic scroll along a section of degree 15, which is of the type described in Proposition 4.10, (iii) and Remark 4.11. Moreover, a special member in the pencil $X_{(\lambda: \mu)}$ is the first infinitesimal neighborhood of the base elliptic quintic scroll. 


\section{APPENDIX}

\section{by C. Ciliberto and K. Hulek}

In this appendix we show that the union $X$ of two quintic elliptic scrolls in $\mathbb{P}^{4}$ intersecting along an elliptic normal curve defines a smooth point in the Hilbert scheme $\mathcal{H}$ of surfaces of degree $d=10$, sectional genus $\pi=6$ and $\chi\left(\mathcal{O}_{X}\right)=0$. The dimension of $\mathcal{H}$ at $[X]$ is 25 . As a corollary we obtain that every (small) deformation of $X$ is again a union of two quintic elliptic scrolls.

Let $E$ be an elliptic normal curve of degree 5 in $\mathbb{P}^{4}$ with origin 0 and non-zero 2-torsion points $\left\{Q_{i} ; i=1,2,3\right\}$. The union of all secants joining points $P$ and $P+Q_{i}$ where $P$ varies in $E$ is a smooth elliptic ruled surface $S_{i}$ of degree 5 . For $i \neq j$ the union

$$
X=S_{i} \cup S_{j} \quad(i \neq j)
$$

is a surface which is singular exactly along $E$ where the two surfaces $S_{i}$ and $S_{j}$ meet transversally. In particular $X$ is a locally complete intersection surface, and it is not difficult to compute that $2 K_{X}=\mathcal{O}_{X}$. As an abstract surface $X$ is a chain of two elliptic ruled surfaces glued along a 2-section. Such surfaces appear in the classification of degenerations of bielliptic surfaces [FM]. Clearly the degree of $X$ is 10. The hyperplane section consists of two elliptic curves intersecting in 5 points, i.e. $\pi(X)=6$. Moreover $q(X)=1, p_{g}(X)=0$, i.e. $\chi\left(\mathcal{O}_{X}\right)=0$.

\section{Theorem 1.}

(i) The Hilbert scheme $\mathcal{H}$ of surfaces of degree 10, sectional genus 6 and $\chi\left(\mathcal{O}_{X}\right)=0$ is smooth at $[X]$.

(ii) The dimension of $\mathcal{H}$ at $[X]$ is 25.

Corollary 2. Every (small) deformation of $X$ is again a union of two quintic elliptic scrolls.

Before we can give a proof of this, we must have a brief look at the geometry of the surfaces $S_{i}$. For this we again fix an elliptic curve $E$ with origin 0 . (This is not the elliptic curve from above, but the curve $E /\left\langle Q_{i}\right\rangle$.) We now denote the non-zero 2-torsion points of $E$ by $\left\{P_{i} ; i=1,2,3\right\}$. The second symmetric product $S^{2} E$ of $E$ is, via the map

$$
\begin{aligned}
\pi: & S^{2} E \rightarrow E \\
\{x, y\} & \mapsto x+y
\end{aligned}
$$

a $\mathbb{P}^{1}$-bundle over $E$. It was already observed in [At, p. 451] that this is the unique indecomposable $\mathbb{P}^{1}$-bundle with $e=-1$. Let $p: E \times E \rightarrow S^{2} E$ be the natural quotient map. The curve

$$
E_{0}=p(E \times\{0\})
$$

is a section of $S^{2} E$. We shall choose the point $p(0,0)$ as its origin and, by abuse of notation, we shall denote it again by 0 . The curves

$$
\Delta_{i}=\left\{\left(x, x+P_{i}\right) ; x \in E\right\}(\cong E) \quad(i=1,2,3)
$$

are mapped 2:1 under $p$ onto 2-sections $E_{i} \subset S^{2} E$. As abstract curves $E_{i}=E /\left\langle P_{i}\right\rangle$. We shall choose the point $0_{i}=p\left(0, P_{i}\right)=p\left(P_{i}, 0\right)$ as the origin of $E_{i}$. The group of 2-torsion points of $\Delta_{i}$ is mapped to two points $\left\{0_{i}, Q_{i}\right\}$. Every fibre $f$ of $S^{2} E$ intersects $E_{i}$ in two 
points which differ by $Q_{i}$. We shall denote the fibre of $S^{2} E$ over $P \in E$ by $f_{P}$, and put $S=S^{2} E$. The following formulae follow immediately from the above description:

$$
\begin{array}{ll}
\text { (1) } & K_{S}=\mathcal{O}_{S}\left(-2 E_{0}+f_{0}\right) \\
\text { (2) } & \left.\mathcal{O}_{S}\left(E_{0}\right)\right|_{E_{0}}=\mathcal{O}_{E_{0}}(0) \\
\text { (3) } & \mathcal{O}_{S}\left(E_{i}\right)=\mathcal{O}_{S}\left(2 E_{0}-f_{P_{i}}\right) \\
\text { (4) } & \left.\mathcal{O}_{S}\left(E_{i}\right)\right|_{E_{i}}=\mathcal{O}_{E_{\boldsymbol{i}}}\left(0_{i}-Q_{i}\right)
\end{array}
$$

The line bundle $\mathcal{O}_{S}(H)=\mathcal{O}_{S}\left(E_{0}+2 f_{0}\right)$ is very ample and embeds $S$ as a scroll of degree 5 into $\mathbb{P}^{4}$. Under this embedding the 2 -sections $E_{i}$ are mapped to quintic elliptic curves and $S$ can be reconstructed from each of these quintic normal curves as translation scrolls defined by the point $Q_{i}$. Conversely fixing $E_{i}$, the three non-zero 2 -torsion points of $E_{i}$ give rise to three elliptic quintic scrolls $S_{i}$. We choose two of them and consider the singular surface $X$ which is the object of our considerations. In order to prove Theorem 1 we have to compute the cohomology groups $H^{i}\left(N_{X}\right)$ of the normal bundle of $X$. For this we first have to investigate the normal bundle of $S$. By [HV, Proposition 4] there is an extension

$$
\text { (5) } 0 \rightarrow K_{S}^{-1} \rightarrow N_{S} \rightarrow \mathcal{O}_{S}(5 H) \otimes K_{S}^{2} \rightarrow 0 .
$$

Lemma 3. (i) $h^{0}\left(N_{S}\right)=25$

(ii) $h^{j}\left(N_{S}\right)=0$ for $j \geq 1$.

Proof. By (1) $K_{S}^{-1}=\mathcal{O}_{S_{i}}\left(2 E_{0}-f_{0}\right)$. It follows, e.g. since $\left.K_{S}^{-1}\right|_{E_{i}} \neq \mathcal{O}_{E_{i}}$ that $h^{0}\left(K_{S_{i}}^{-1}\right)=0$. Clearly $h^{2}\left(K_{S}^{-1}\right)=h^{0}\left(K_{S}^{2}\right)=0$ and hence by Riemann-Roch $h^{1}\left(K_{S}^{-1}\right)=0$. Let $\mathcal{M}=$ $\mathcal{O}_{S}(5 H) \otimes K_{S}^{2}$. From the definition of $H$ and (1) one obtains that $\mathcal{M}=\mathcal{O}_{S}\left(E_{0}+12 f_{0}\right)$. It is easy to see by the Nakai-Moishezon criterion that $\mathcal{M} \otimes K_{S}^{-1}=\mathcal{O}_{S}\left(3 E_{0}+11 f_{0}\right)$ is ample and hence $h^{j}(\mathcal{M})=0$ for $j \geq 1$. But then Riemann-Roch gives $h^{0}(\mathcal{M})=25$ and the assertions follow from sequence $(5)$.

Lemma 4. $h^{j}\left(N_{S}\left(-E_{i}\right)\right)=0$ for $j \geq 0$.

Proof. Again setting $\mathcal{M}=\mathcal{O}_{S}(5 H) \otimes K_{S}^{2}$ and twisting (5) by $\mathcal{O}_{S}\left(-E_{i}\right)$ we obtain

$$
\text { (6) } 0 \rightarrow K_{S}^{-1}\left(-E_{i}\right) \rightarrow N_{S}\left(-E_{i}\right) \rightarrow \mathcal{M}\left(-E_{i}\right) \rightarrow 0 \text {. }
$$

Since $K_{S}^{-1}\left(-E_{i}\right)=\mathcal{O}_{S}\left(f_{P_{i}}-f_{0}\right)$ this line bundle has no cohomology. By definition of $\mathcal{M}$ and (3) we obtain $\mathcal{M}\left(-E_{i}\right)=\mathcal{O}_{S}\left(-E_{0}+12 f_{0}+f_{P_{i}}\right)$. Clearly $h^{0}\left(\mathcal{M}\left(-E_{i}\right)\right)=h^{2}\left(\mathcal{M}\left(-E_{i}\right)\right)=0$ and once again by Riemann-Roch $h^{1}\left(\mathcal{M}\left(-E_{i}\right)\right)=0$. The assertion now follows from the above sequence (6).

We can now compute the cohomology of $N_{X}$. Theorem 1 is an immediate consequence of Proposition 5. (i) $h^{0}\left(N_{X}\right)=25$

(ii) $h^{j}\left(N_{X}\right)=0$ for $j \geq 1$.

Proof. Let $C=E_{i}$ and consider $T=N_{C / S_{i}} \otimes N_{C / S_{j}}=\mathcal{O}_{C}\left(20_{i}-Q_{i}-Q_{j}\right)$. In particular $T$ is a non-trivial element of order 2. As in [CLM, section 2.2] we have exact sequences

$$
\text { (7) }\left.0 \rightarrow N_{S_{i}} \rightarrow N_{X}\right|_{S_{i}} \rightarrow T \rightarrow 0
$$

$$
\left.\left.0 \rightarrow N_{X}\right|_{S_{j}} \otimes \mathcal{O}_{S_{j}}(-C) \rightarrow N_{X} \rightarrow N_{X}\right|_{S_{i}} \rightarrow 0
$$


It follows from Lemma 3 and sequence (7) that $h^{0}\left(\left.N_{X}\right|_{S_{i}}\right)=25$ and $h^{j}\left(\left.N_{X}\right|_{S_{i}}\right)=0$ for $j \geq 1$. Twisting $(7)$ by $\mathcal{O}_{S_{i}}(-C)$ we get

$$
\text { (9) }\left.\quad 0 \rightarrow N_{S_{i}}(-C) \rightarrow N_{X}\right|_{S_{i}} \otimes \mathcal{O}_{S_{i}}(-C) \rightarrow T \otimes \mathcal{O}_{S_{i}}(-C) \rightarrow 0
$$

Using (3) we find that $T \otimes \mathcal{O}_{S_{i}}(-C)=\mathcal{O}_{C}\left(0_{i}-Q_{j}\right)$ which again has no cohomology. It therefore follows from Lemma 4 that $h^{j}\left(\left.N_{X}\right|_{S_{i}} \otimes \mathcal{O}_{S_{i}}(-C)\right)=0$ for $j \geq 0$. The claim then follows from sequence (8).

Proof of Corollary 2. Let $X(2,5)$ be the modular curve parametrizing elliptic curves with a level 5 structure and a non-zero 2-torsion point. We claim that every point of $X(2,5)$ which is not a cusp gives rise to some $X$ as before. Indeed the elliptic curves with level 5 structure are in 1:1 correspondence with Heisenberg invariant elliptic normal curves in $\mathbb{P}^{4}$. Given a non-zero 2 -torsion point $P$ we have exactly 2 other non-zero 2 -torsion points. Hence we can use these 2 points to construct $X$. Now consider some $X$ as before. Up to a change of coordinates we can assume that $X$ is constructed as the union of two translation scrolls of a Heisenberg invariant elliptic normal curve. Let $\mathcal{H}_{0}$ be the unique component of $\mathcal{H}$ containing $X$. We have a map $\Phi: X^{0}(2,5) \times$ PGL $(5, \mathbb{C}) \rightarrow \mathcal{H}_{0}$ where $X^{0}(2,5)$ is $X(2,5)$ without the cusps. Since every elliptic normal curve has only finitely many automorphisms $\Phi$ is locally 1:1 and hence a local (analytic) isomorphism. This proves the claim.

Clearly this situation can be generalized to higher dimensions in several ways. We hope to return to this in the near future.

\section{REFERENCES}

[ADHPR] Aure, A.B., Decker, W., Hulek, K., Popescu, S., Ranestad, K., The Geometry of Bielliptic Surfaces in $\mathbb{P}^{4}$, Int. J. of Math. 4 (1993), 873-902.

[At] Atiyah, M.F., Vector bundles over an elliptic curve, Proc. Lond. Math. Soc.(3) VII (1957), 414-452.

[Au] Aure, A.B., Surfaces on quintic threefolds associated to the Horrocks-Mumford bundle, LNM 1399 (1989), 1-9.

[Bei] Beilinson, A., Coherent sheaves on $\mathbb{P}^{N}$ and problems of linear algebra, Funct. Anal. Appl. 12 (1978), 214-216.

[BH] Barth, W., Hulek, K., Projective models of Shioda modular surfaces, Manuscripta Math. 50 (1985), 73-132.

[BHM1] Barth, W., Hulek, K., Moore, R., Shioda's modular surface S(5) and the Horrocks-Mumford bundle, Vector bundles on algebraic varieties. Papers presented at the Bombay colloquim 1984, Oxford University Press, Bombay, 1987, pp. 35-106.

[BHM2] Barth, W., Hulek, K., Moore, R., Degenerations of Horrocks-Mumford surfaces, Math. Ann. 277 (1987), 735-755.

[Bi] Bianchi, L., Ueber die Normsalformrn dritter und fünfter Stufe des elliptischen Integrals erster Gattung, Math. Ann. 17 (1880), 234-262.

[BL] Birkenhake, C., Lange, H., Fixed point free automorphisms of abelian varieties, Geom. Dedicata 51 (1994), 201-213.

[BM] Barth, W., Moore, R., Geometry in the space of Horrocks-Mumford surfaces, Topology 28 (1989), 231-245.

[CC] Catanese, F., Ciliberto, C., Symmetric products of elliptic curves and surfaces of general type with $p_{g}=q=1$, Preprint, Rome 1990 .

[CLM] Ciliberto, C., Lopez, A., Miranda, R., Projective degenerations of K3 surfaces, Gaussian maps, and Fano threefolds, Invent. Math 114 (1993), 641-667.

[De1] Decker, W., Das Horrocks-Mumford-Bündel und das Modul-Schema für stabile 2-Vektorbündel über $\mathbb{P}_{4}$ mit $c_{1}=-1, c_{2}=4$., Math. Z. 188 (1984), 101-110. 
[De2] Monads and cohomology modules of rank 2 vector bundles, Compositio Math. 76 (1990), $7-17$.

[DES] Decker, W., Ein, L., Schreyer, F.-O., Construction of surfaces in $\mathbb{P}^{4}$, J. of Algebraic Geometry 2 (1993), 185-237.

[EP] Ellingsrud, G., Peskine, D., Sur les surfaces lisses de $\mathbb{P}_{4}$, Inv. Math. 95 (1989), 1-12.

[Fe] Ferrand, D., Courbes gauches et fibrés de rang 2, C. R. Acad. Sci. Paris(A) 281 (1975), 345-347.

[FM] Friedman, R., Morrison, D.R.(eds), The birational geometry of degenerations, PM 29, Birkhäuser, Boston, 1983.

[Go] Gotzmann, G., Eine Bedingung für die Flachheit und das Hilbertpolynom eines graduierten Ringes, Math. Z. 158 (1978), 61-70.

[GP1] Gross, M., Popescu, S., Equations of $(1, d)$-polarized abelian surfaces (to appear).

[GP2] Gross, M., Popescu, S., Calabi-Yau threefolds and moduli of abelian surfaces (to appear).

[Gr] Green, M., Koszul Homology and the geometry of projective varieties, J. Diff. Geom. 19 (1984), 125-171.

[Ha] Hartshorne, R., Connectedness of the Hilbert scheme, Inst. Hautes Études Sci. Publ. Math. 29 (1966), 5-48.

[HCV] Hilbert, D., Cohn-Vossen, S., Geometry and the Imagination, Chelsea Pub. Co., New York, 1952.

[HKW] Hulek, K., Kahn, C., Weintraub, S., Moduli Spaces of Abelian Surfaces: Compactification, Degenerations, and Theta Functions., Walter de Gruyter, Berlin, 1993.

[HM] Horrocks, G., Mumford, D., A rank 2 vector bundle on $\mathbb{P}^{4}$ with 15,000 symmetries, Topology 12 (1973), 63-81.

[Hu1] Hulek, K., Projective geometry of elliptic curves, Astérisque 137 (1986).

[Hu2] Hulek, K., Geometry of the Horrocks-Mumford bundle, Algebraic Geometry, Bowdoin 1985, Proc. Symp. Pure Math., vol. 46, 1987, pp. 69-85.

[HV] Hulek, K., Van de Ven, A., The Horrocks-Mumford bundle and the Ferrand construction, Manuscripta Math. 50 (1985), 313-335.

[Kl] Klein, F., Vorlesungen über das Ikosaeder und die Auflösungen der Gleichungen von fünftem Grade, Kommentiert und herausgegeben von P. Slodowy, Birkhäuser, 1992.

[LB] Lange, H., Birkenhake, C., Complex abelian varieties, Springer, Berlin, Heidelberg, 1992.

[Ma1] Manolache N., On the normal bundle to abelian surfaces embedded in $\mathbb{P}^{4}(\mathbb{C})$, Manuscripta Math. 55 (1986), 111-119.

[Ma2] - Cohen-Macaulay nilpotent structures, Rev. Roum. Math. Pures et Appl. 31 (1986), 563-575.

[Ma3] $\longrightarrow$ Syzygies of abelian surfaces embedded in $\mathbb{P}^{4}(\mathbb{C})$, J. reine und angew. Math. 384 (1988), $180-191$.

[Ma4] - The equations of the abelian surfaces embedded in $\mathbb{P}^{4}(\mathbb{C})$, J. reine und angew. Math. 394 (1989), 196-202.

[Mac] Bayer, D., Stillman, M., Macaulay: A system for computation in algebraic geometry and commutative algebra, Source and object code available for Unix and Macintosh computers. Contact the authors, or download from math.harvard.edu via anonymous ftp..

[Moo] Moore, R., Heisenberg-invariant quintic 3-folds, and sections of the Horrocks-Mumford bundle, Preprint, Canberra 1985.

[Mor1] Morrison, D., Semistable degenerations of Enriques and hyperelliptic surfaces, Thesis, Harvard 1980.

[Mor2] Morrison, D., Semistable degenerations of Enriques and hyperelliptic surfaces, Duke Math. J. 231 (1981), 197-249.

[Na] Naruki, I., Über die Kleinsche Ikosaeder-Kurve sechsten Grades, Math. Ann. 231 (1978), 205216.

[Ok1] Okonek, C., Moduli reflexiver Garben und Flächen von kleinem Grad in $\mathbb{P}^{4}$, Math. Z. 184 (1983), 549-572.

[Ok2] Leflexive Garben auf $\mathbb{P}^{4}$, Math. Ann. 269 (1982), 211-237.

[Po] Popescu, S., On smooth surfaces of degree $\geq 11$ in the projective fourspace, Thesis, Saarbrücken 1993.

[PS] Peskine, Ch., Szpiro, L., Liaison des variétés algébriques I, Invent. Math. 26 (1974), 271-302.

[Ra] Ranestad, K., On smooth surfaces of degree ten in the projective fourspace, Thesis, Oslo 1988. 
[Rei] Reider, I., Vector bundles of rank 2 linear systems on algebraic surfaces, Ann. Math. 127 (1988), 309-316.

[Sch] Schreyer, F.-O., Small fields in constructive algebraic geometry, Moduli of Vector Bundles, 35-th. Taniguchi International Symposium, ed. M. Maruyama, Lecture Notes in Pure and Appl. Mathematics Series, vol. 179, Marcel Dekker, New York, 1996.

[Seg] Segre, C., Sull' Incidenza di rette e piani nello spazio a quattro dimensioni, Rend. Palermo II (1888), 42-52.

[Sem] Semple, J.G., Cremona transformations of space of four dimensions by means of quadrics and the reverse transformations, Phil. Trans. Royal Soc. London, Series A 228 (1929), 331-376.

[Ser] Serrano, F., Divisors of bielleptic surfaces and embeddings in $\mathbb{P}^{4}$, Math. Z. 203 (1990), 527-533.

[Sh] Shioda, T., On elliptic modular surfaces, J. Math. Soc. Japan 24 (1972), 20-59.

[So] Sommese, A.J., Hyperplane sections of projective surfaces I. The adjunction mapping, Duke Math. J. 46 (1979), 377-401.

[SV] Sommese, A.J., Van de Ven, A., On the adjunction mapping, Math. Ann. 278 (1987), 593-603.

WOLFRAM DECKER

Fachbereich Mathematik, Universität des SaArlandes, D 66041 SaARbrücken, Germany

E-mail address: decker@math.uni-sb.de

Klaus Hulek

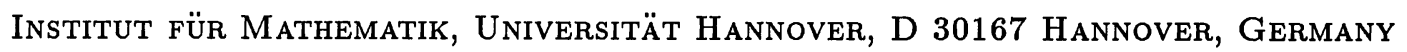

E-mail address: hulek@math.uni-hannover.de

SORIN POPESCU

Mathematics Department, Brandeis University, Waltham, MA 02254, USA

E-mail address: psorin@math.harvard.edu

KRISTIAN RANESTAD

Matematisk Institutt, Universitetet i Oslo, Box 1053 Blindern, N-0316 Oslo 3, Norway

E-mail address: ranestad@math.uio.no 